\title{
Adaptive Near-Optimal \\ Rank Tensor Approximation for High-Dimensional Operator Equations
}

Markus Bachmayr and Wolfgang Dahmen

Bericht Nr. 363

April 2013

Key words: Low-rank tensor approximation, adaptive methods, high-dimensional operator equations, computational complexity

Math. Subject Classifications: 41A46, 41A63, 65D99, 65J10, 65N12, $65 \mathrm{~N} 15$

Institut für Geometrie und Praktische Mathematik RWTH Aachen

Templergraben 55, D-52056 Aachen (Germany) 


\title{
Adaptive Near-Optimal Rank Tensor Approximation for High-Dimensional Operator Equations
}

\author{
Markus Bachmayr and Wolfgang Dahmen \\ Institut für Geometrie und Praktische Mathematik, RWTH Aachen, Germany \\ email: bachmayr@igpm.rwth-aachen.de, dahmen@igpm.rwth-aachen.de
}

April 29, 2013

\begin{abstract}
We consider a framework for the construction of iterative schemes for operator equations that combine low-rank approximation in tensor formats and adaptive approximation in a basis. Under fairly general assumptions, we obtain a rigorous convergence analysis, where all parameters required for the execution of the methods depend only on the underlying infinite-dimensional problem, but not on a concrete discretization. Under certain assumptions on the rates for the involved low-rank approximations and basis expansions, we can also give bounds on the computational complexity of the iteration as a function of the prescribed target error. Our theoretical findings are illustrated and supported by computational experiments. These demonstrate that problems in very high dimensions can be treated with controlled solution accuracy.

Keywords: Low-rank tensor approximation, adaptive methods, high-dimensional operator equations, computational complexity

Mathematics Subject Classification (2000): 41A46, 41A63, 65D99, 65J10, $65 \mathrm{~N} 12,65 \mathrm{~N} 15$
\end{abstract}

\section{Introduction}

\subsection{Motivation}

Any attempt to recover or approximate a function of a large number of variables with the aid of classical low-dimensional techniques is inevitably impeded by the curse of dimensionality. This means that, when only assuming classical smoothness (e.g. in terms of Sobolev or Besov regularity) of order $s>0$, the necessary computational work needed to realize a desired target accuracy $\varepsilon$ in $d$ dimensions scales like $\varepsilon^{-d / s}$, i.e., one faces an exponential increase in the spatial dimension $d$. This can be ameliorated by dimensiondependent smoothness measures. In many high-dimensional problems of interest, the approximand has bounded high-order mixed derivatives, which under suitable assumptions can be used to construct sparse grid-type approximations where the computational work scales like $C_{d} \varepsilon^{-1 / s}$. Under such regularity assumptions, one can thus obtain a convergence rate independent of $d$. In general, however, the constant $C_{d}$ will still grow exponentially in $d$. This has been shown to hold even under extremely restrictive smoothness assumptions in [27], and has been observed numerically in a relatively simple but realistic example in $[12$.

Hence, in contrast to the low-dimensional regime, regularity is no longer a sufficient structural property that ensures computational feasibility, and further low-dimensional 
structure of the sought high-dimensional object is required. Such a structure could be the dependence of the function on a much smaller (unknown) number of variables, see e.g. [11]. It could also mean sparsity with respect to some (a priori) unknown dictionary. In particular, dictionaries comprized of rank-one tensors $g\left(x_{1}, \ldots, x_{d}\right)=g_{1}\left(x_{1}\right) \cdots g_{d}\left(x_{d}\right)=$ : $\left(g_{1} \otimes \cdots \otimes g_{d}\right)(x)$ open very promising perspectives and have recently attracted substantial attention.

As a simple example consider $g(x)=\bigotimes_{i=1}^{d} g_{i}\left(x_{i}\right)$ on the unit cube $\Omega=[0,1]^{d}$, where the $g_{i}$ are sufficiently smooth. Employing for each factor $g_{i}$ a standard spline approximation of order $s$ with $n$ knots yields an $L_{\infty}$-accuracy of order $n^{-s}$, which gives rise to an overall accuracy of the order of $d n^{-s}$ at the expense of $d n=: N$ degrees of freedom. Hence, assuming that $\|g\|_{\infty}$ does not depend on $d$, an accuracy $\varepsilon$ requires

$$
N=N(\varepsilon, d) \sim d^{\frac{1-s}{s}} \varepsilon^{-1 / s}
$$

degrees of freedom. In contrast, it would take the order of $N=n^{d}$ degrees of freedom to realize an accuracy of order $n^{-s}=N^{-d / s}$ when using a standard tensor product spline approximation, which means that in this case $N(\varepsilon, d) \sim \varepsilon^{-d / s}$. Thus, while the first approximation - using a nonlinear parametrization of a reference basis - breaks the curse of dimensionality, the second one obviously does not.

Of course, $u$ being a simple tensor is in general an unrealistic assumption, but the curse of dimensionality can still be significantly mitigated when $f$ is well approximable by relatively short sums of rank-one tensors. By this we mean that for some norm $\|\cdot\|$ we have

$$
\left\|u-\sum_{j=1}^{r(\varepsilon)} g_{1, j} \otimes \cdots \otimes g_{j, d}\right\| \leq \varepsilon
$$

where the rank $r(\varepsilon)$ grows only moderately as $\varepsilon$ decreases. In our initial example, in these terms we had $r(\varepsilon)=1$ for all $\varepsilon>0$. Assuming that all the factors $g_{j, i}$ in the above approximation are sufficiently smooth, the count (1) applied to each summand with target accuracy $\varepsilon / r$ shows that now at most

$$
N(\varepsilon, d, r) \lesssim r^{1+\frac{1}{s}} d^{\frac{1-s}{s}} \varepsilon^{-\frac{1}{s}}
$$

degrees of freedom are required, which is still acceptable. This is clearly a very crude reasoning because it does not take a possible additional decay in the rank-one summands into account.

This argument, however, already indicates that good approximability in the sense of (2) is not governed by classical regularity assumptions. Instead, the key is to exploit an approximate global low-rank structure of $u$. This leads to a highly nonlinear approximation problem, where one aims to identify suitable lower-dimensional tensor factors, which can be interpreted as a $u$-dependent dictionary.

This discussion, although admittedly somewhat oversimplified, immediately raises several questions which we will briefly discuss as they guide subsequent developments.

Format of approximation: The hope that $r(\varepsilon)$ in (2) can be rather small is based on the fact that the rank-one tensors are allowed to "optimally adapt" to the approximand $u$. The format of the approximation used in (2) is sometimes called canonical since it is a formal direct generalization of classical Hilbert Schmidt expansions for $d=2$. However, a closer look reveals a number of well-known pitfalls. In fact, they are already encountered in the discrete case. The collection of sums of ranks one tensors of a given length is not closed, and the best approximation problem is not well-posed, see e.g. [10]. There 
appears to be no reliable computational strategy that can be proven to yield near-minimal rank approximations for a given target accuracy in this format. In this work, we therefore employ different tensor formats that allow us to obtain provably near-minimal rank approximations, as explained later.

A two-layered problem: Given a suitable tensor format, even if a best tensor approximation is known in the infinite-dimensional setting of the continous problem, the resulting lower-dimensional factors still need to be approximated. Since finding these factors is part of the solution process, the determination of efficient discretizations for these factors will need to be intertwined with the process of finding low-rank expansions. We have chosen here to organize this process through selecting low-dimensional orthonormal wavelet bases for the tensor factors. However, other types of basis expansions would be conceivable as well.

The issue of the total complexity of tensor approximations, taking the approximation of the involved lower-dimensional factors into account, is addressed in [17,31.

\subsection{Conceptual Preview}

The problem of finding a suitable format of tensor approximations has been extensively studied in the literature over that past years, however, mainly in the discrete or finitedimensional setting, see e.g. [15, 20, 22, 28, 30]. Some further aspects in a function space setting have been addressed e.g. in [13, 35, 36]. For an overview and further references we also refer to [18 and the recent survey [16]. A central question in these works is: given a tensor, how can one in a stable manner obtain low-rank approximations, and how accurate are they when compared with best tensor approximations in the respective format?

We shall heavily draw on these findings in the present paper, but under the following somewhat different perspectives. First of all, we are interested in the continuous infinitedimensional setting, i.e., in sparse tensor approximations of a function which is a priori not given in any finite tensor format but which one may expect to be well approximable by simple tensors in a way to be made precise later. We shall not discuss here the question under which concrete conditions this is actually the case. Moreover, the objects to be recovered are not given explicitly but only implicitly as a solution to an operator equation

$$
A u=f,
$$

where $A: V \rightarrow V^{\prime}$ is an isomorphism of some Hilbert space $V$ onto its dual $V^{\prime}$. One may think of $V$, in the simplest instance, as a high-dimensional $\mathrm{L}_{2}$ space, or as a Sobolev space. More generally, as in the context of parametric diffusion problems, $V$ could be a tensor product of a Sobolev space and an $\mathrm{L}_{2}$ space. Accordingly, we shall always assume that we have a Gelfand triplet

$$
V \subset H \equiv H^{\prime} \subset V^{\prime},
$$

in the sense of dense continuous embeddings, where we assume that $H$ is a tensor product Hilbert space, that is,

$$
H=H_{1} \otimes \cdots \otimes H_{d}
$$

with lower-dimensional Hilbert spaces $H_{i}$. A typical example would be $H=\mathrm{L}_{2}\left(\Omega^{d}\right)=$ $\mathrm{L}_{2}(\Omega) \otimes \cdots \otimes \mathrm{L}_{2}(\Omega)$ for a domain $\Omega$ of small spatial dimension.

The main contribution of this work is to put forward a strategy that addresses the main obstacles identified above and results in an algorithm which, under mild assumptions, can be rigorously proven to provide for any target accuracy $\varepsilon$ an approximate solution of nearminimal rank and representation complexity of the involved tensor factors. Specifically, (i) 
it is based on stable tensor formats relying on optimal subspaces; (ii) successive solution updates involve a combined refinement of ranks and factor discretizations; (iii) (near)optimality is achieved, thanks to (i), through accompanying suitable subspace correction and coarsening schemes.

The following comments on the main ingredients are to provide some orientation. A first essential step is to choose a universal basis for functions of a single variable in $H_{i}$. Here, we focus on wavelet bases, but other systems like the trigonometric system for periodic problems are conceivable as well. As soon as functions of a single variable, especially the factors in our rank-one tensors, are expanded in such a basis, the whole problem of approximating $u$ reduces to approximating its infinite coefficient tensor $\mathbf{u}$ induced by the expansion

$$
u=\sum_{\nu \in \nabla^{d}} u_{\nu} \Psi_{\nu}, \quad \Psi_{\nu}:=\psi_{\nu_{1}} \otimes \cdots \otimes \psi_{\nu_{d}}, \quad \mathbf{u}=\left(u_{\nu}\right)_{\nu \in \nabla^{d}},
$$

see below. The original operator equation (4) is then equivalent to an infinite system

$$
\mathbf{A u}=\mathbf{f}, \quad \text { where } \quad \mathbf{A}=\left(\left\langle A \Psi_{\nu}, \Psi_{\nu^{\prime}}\right\rangle\right)_{\nu, \nu^{\prime} \in \nabla^{d}}, \mathbf{f}=\left(\left\langle f, \Psi_{\nu}\right\rangle\right)_{\nu \in \nabla^{d}} .
$$

For standard types of Sobolev spaces $V$ it is well understood how to rescale the tensor product basis $\left\{\Psi_{\nu}\right\}_{\nu \in \nabla^{d}}$ in such a way that it becomes a Riesz basis for $V$. This, in turn, together with the fact that $\kappa_{V \rightarrow V^{\prime}}(A):=\|A\|_{V \rightarrow V^{\prime}}\left\|A^{-1}\right\|_{V^{\prime} \rightarrow V}$ is finite, allows one to show that $\kappa_{\ell_{2} \rightarrow \ell_{2}}(\mathbf{A})$ is finite. Hence one can find a positive $\omega$ such that $\|\mathbf{I}-\omega \mathbf{A}\|_{\ell_{2} \rightarrow \ell_{2}} \leq \rho<1$, i.e., the operator $\mathbf{I}-\omega \mathbf{A}$ is a contraction so that the iteration

$$
\mathbf{u}_{k+1}:=\mathbf{u}_{k}+\omega\left(\mathbf{f}-\mathbf{A} \mathbf{u}_{k}\right), \quad k=0,1,2, \ldots,
$$

converges for any initial guess to the solution $\mathbf{u}$ of 7 ).

Of course, (8) is only an idealization because the full coefficient sequences $\mathbf{u}_{k}$ cannot be computed. Nevertheless, adaptive wavelet methods can be viewed as realizing (8) approximately, keeping possibly few wavelet coefficients "active" while still preserving enough accuracy to ensure convergence to $\mathbf{u}$ (see e.g. 8,9]).

In the present high-dimensional context this kind of adaptation is no longer feasible. Instead, we propose here a "much more nonlinear" adaptation concept. Being able to keep increasingly accurate approximations on a path towards near-minimal rank approximations with properly sparsified tensor factors relies crucially on suitable correction mechanisms. An important contribution of this work is to identify and analyze just such methods. Conceptually, they are embedded in a properly perturbed numerical realization of (8) of the form

$$
\mathbf{u}_{k+1}=\mathrm{C}_{\varepsilon_{3}(k)}\left(\mathrm{P}_{\varepsilon_{2}(k)}\left(\mathbf{u}_{k}+\omega\left(\mathbf{f}-\mathbf{A} \mathbf{u}_{k}\right)\right)\right), \quad k=0,1,2, \ldots,
$$

where $\mathrm{P}_{\varepsilon_{i}(k)}, i=1,2, \mathrm{C}_{\varepsilon_{3}(k)}$ are certain reduction operators and the $\varepsilon_{i}(k), i=1,2,3$, are suitable tolerances which decrease for increasing $k$.

More precisely, the purpose of $\mathrm{P}_{\varepsilon}$ is to "correct" the current tensor expansion and, in doing so, reduce the rank subject to an accuracy tolerance $\varepsilon$. We shall always refer to such a rank reduction operation as a recompression. For this operation to work as desired, it is essential that the employed tensor format is stable in the sense that the best approximation problem for any given ranks is well-posed. As explained above, this excludes the use of the canonical format. Instead we use the so-called hierarchical Tucker (HT) format, since on the one hand it inherits the stability of the Tucker format [13], as a classical best subspace method, while on the other hand it better ameliorates the curse of 
dimensionality that the Tucker format may still be prone to. In $\$ 2$ we collect the relevant prerequisites. This draws to a large extent on known results for the finite-dimensional case, but requires proper formulation and extension of these notions and facts for the current sequence space setting. The second reduction operation $\mathrm{C}_{\epsilon}$, in turn, is a coarsening scheme that reduces the number of degrees of freedom used by the wavelet representations of the tensor factors, again subject to some accuracy constraint $\epsilon$.

\subsection{What is New?}

The use of rank reduction techniques in iterative schemes is in principle not new, see e.g. $3,5,6,19,21,23,25$ and the further references given in [16]. To our knowledge, corresponding approaches can be subdivided roughly into two categories. In the first one, iterates are always truncated to a fixed tensor rank. This allows one to control the complexity of the approximation, but convergence of such iterations can be guaranteed only under very restrictive assumptions (e.g. concerning highly effective preconditioners). In the second category, schemes achieve a desired target accuracy by instead prescribing an error tolerance for the rank truncations, but the corresponding ranks arising during the iteration are not controlled. A common feature of both groups of results is that they operate on a fixed discretization of the underlying continuous problems.

In contrast, the principal novelty of the present approach can be sketched as follows. The first key element is to show that based on a known error bound for a given approximation to the unknown solution, a judiciously chosen recompression produces a near-minimal rank approximation to the solution of the continous problem for a slightly larger accuracy tolerance. Moreover, the underlying projections are stable with respect to certain sparsity measures. As pointed out before, this reduction needs to be intertwined with a sufficiently accurate but possibly coarse approximation of the tensor factors. A direct coarsening of the full wavelet coefficient tensor would face the curse of dimensionality, and thus would be practically infeasible. The second critical element is therefore to introduce certain lowerdimensional quantities, termed tensor contractions, from which the degrees of freedom to be discarded in the coarsening are identified. This notion of contractions also serves to define suitable sparsity classes with respect to wavelet coefficients, facilitating a computationally efficient, rigorously founded combination of tensor recompression and coefficient coarsening.

These concepts culminate in the main result of this paper, which can be summarized in an admittedly oversimplified way as follows.

Meta-Theorem: Whenever the solution to (7) has certain tensor-rank approximation rates and when the involved tensor factors have certain best $N$-term approximation rates, then a judicious numerical realization of the iteration (9) realizes these rates. Moreover, up to logarithmic factors, the computational complexity is optimal. More specifically, for the smallest $n$ such that the approximate solution $\mathbf{u}_{k}$ satisfies $\left\|\mathbf{u}_{k}-\mathbf{u}\right\|_{\ell_{2}} \leq \tau, \mathbf{u}_{k}$ has HT-ranks that can be bounded by a uniformly constant multiple of the smallest possible $H T$-ranks needed to realize accuracy $\tau$.

To our knowledge this is the first result of this type, where convergence to the solution of the infinite-dimensional problem is guaranteed under realistic assumptions, and all ranks arising during the process remain proportional to the respective smallest possible ones. A rigorous proof of rank near optimality, using an iteration of the above type, is to be contrasted to approaches based a greedy approximation as employed in connection with the so-called PGD (proper generalized decomposition), see e.g. [14]. The latter scheme aims 
at constructing an approximation in the (unstable) canonical format through successive greedy updates. This does, in principle, not seem to offer much hope for finding minimal or near-minimal rank approximations, as the greedy search operates far from orthonormal bases, and errors committed early in the iteration cannot easily be corrected.

\subsection{Layout}

The remainder of the paper is devoted to the development of the ingredients and their complexity analysis needed to make the statements in the above Meta-Theorem precise. Trying to carry out this program raises some issues which we will briefly address now, as they guide the subsequent developments.

After collecting in $\$ 2$ some preliminaries, $\$ 3$ is devoted to a pivotal element of our approach, namely the development and analysis of suitable recompression and coarsening schemes that yield an approximation in the HT-format that is, for a given target accuracy, of near-minimal rank with possibly sparse tensor factors (in a sense to be made precise later).

Of course, one can hope that the solution of (4) is particularly tensor sparse in the sense that relatively low HT-ranks already provide high accuracy if the data $f$ are tensor sparse, and if the operator $A$ (resp. A) is tensor sparse in the sense that its application does not increase ranks too drastically. Suitable models of operator classes that allow us to properly weigh tensor sparsity and wavelet expansion sparsity are introduced and analyzed in $\$ 4$. The approximate application of such operators with certified output accuracy builds on the findings in $\$ 3$.

Finally, in $\$ 5$ we formulate an adaptive iterative algorithm and analyze its complexity. Starting from the coarsest possible approximation $\mathbf{u}^{0}=0$, approximations in the tensor format are built successively, where the error tolerances in the iterative scheme are updated for each step in such a way that two goals are achieved. On the one hand, the tolerances are sufficiently stringent to guarantee the convergence of the iteration up to any desired target accuracy. On the other hand, we ensure that at each stage of the iteration, the approximations remain sufficiently coarse to realize the Meta-Theorem formulated above. Here we specify concrete tensor approximability assumptions on $\mathbf{u}, \mathbf{f}$ and $\mathbf{A}$ that allow us to make its statement precise.

\section{Preliminaries}

In this section we set the notation and collect the relevant ingredients for stable tensor formats in the infinite-dimensional setting. In the remainder of this work, we shall use for simplicity the abbreviation $\|\cdot\|:=\|\cdot\|_{\ell_{2}}$, with the $\ell_{2}$-space on the appropriate index set.

Our basic assumption is that we have a Riesz basis $\left\{\Psi_{\nu}\right\}_{\nu \in \nabla^{d}}$ for $V$, where $\nabla$ is a countable index set. In other words, we require that the index set has Cartesian product structure. Therefore any $u \in V$ can be identified with its basis coefficient sequence $\mathbf{u}:=\left(\left\langle u, \Psi_{\nu}\right\rangle\right)_{\nu \in \nabla^{d}} \in \ell_{2}\left(\nabla^{d}\right)$ with uniformly equivalent norms. Thus, $d$ will in general correspond to the spatial dimension of the domain of functions under consideration. In addition it can be important to reserve the option of grouping some of the variables in a possibly smaller number $m \leq d$ of portions of variables, i.e., $m \in \mathbb{N}$ and $d=d_{1}+\ldots+d_{m}$ for $d_{i} \in \mathbb{N}$.

A canonical point of departure for the construction of $\left\{\Psi_{\nu}\right\}$ is a collection of Riesz bases for each component Hilbert space $H_{i}$ (see (6)), which we denote by $\left\{\psi_{\nu}^{H_{i}}\right\}_{\nu \in \nabla^{H_{i}}}$. To fit in the above context, we may assume without loss of generality that all $\nabla^{H_{i}}$ are 
identical, denoted by $\nabla$. The precise structure of $\nabla$ is irrelevant at this point; however, in the case that the $\psi_{\nu}^{H_{i}}$ are wavelets, each $\nu=(j, k)$ encodes a dyadic level $j=|\nu|$ and a spatial index $k=k(\nu)$. This latter case is of particular interest, since for instance when $V$ is a Sobolev space, a simple rescaling of $\psi_{\nu_{1}}^{H_{1}} \otimes \cdots \otimes \psi_{\nu_{d}}^{H_{d}}$ yields a Riesz basis $\left\{\Psi_{\nu}\right\}$ for $V \subseteq H$ as well.

A simple scenario would be $V=H=\mathrm{L}_{2}\left([0,1]^{d}\right)$, which is the situation considered in our numerical illustration in 86 . A second example are elliptic diffusion equations with stochastic coefficients. In this case, $V=\mathrm{H}_{0}^{1}(\Omega) \otimes \mathrm{L}_{2}\left([-1,1]^{\infty}\right)$, and $H=\mathrm{L}_{2}\left(\Omega \times[-1,1]^{\infty}\right)$. Here a typical choice of bases for $\mathrm{L}_{2}\left([-1,1]^{\infty}\right)$ are tensor products of polynomials on $[-1,1]$, while one can take a wavelet basis for $\mathrm{H}_{0}^{1}(\Omega)$, obtained by rescaling a standard $\mathrm{L}_{2}$ basis. A third representative scenario concerns diffusion equations on high-dimensional product domains $\Omega^{d}$. Here, for instance, $V=\mathrm{H}^{1}\left(\Omega^{d}\right)$ and $H=\mathrm{L}_{2}\left(\Omega^{d}\right)$. We shall comment on some additional difficulties that arise in the application of operators in this case in Remark 15.

We now regard $\mathbf{u}$ as a tensor of order $m$ on $\nabla^{d}=\nabla^{d_{1}} \times \cdots \times \nabla^{d_{m}}$. Rather than looking for approximations or representations in the canonical format

$$
\mathbf{u}=\sum_{k=1}^{r} a_{k} \mathbf{U}_{k}^{(1)} \otimes \cdots \otimes \mathbf{U}_{k}^{(d)}
$$

we consider tensor representations of the form

$$
\mathbf{u}=\sum_{k_{1}=1}^{r_{1}} \cdots \sum_{k_{m}=1}^{r_{m}} a_{k_{1}, \ldots, k_{m}}\left(\mathbf{U}_{k_{1}}^{(1)} \otimes \cdots \otimes \mathbf{U}_{k_{m}}^{(m)}\right) .
$$

Here the order- $m$ tensor $\mathbf{a}=\left(a_{k_{1}, \ldots, k_{m}}\right)_{1 \leq k_{i} \leq r_{i}: i=1, \ldots, m}$ is referred to as core tensor. The matrix $\mathbf{U}^{(i)}=\left(\mathbf{U}_{\nu_{i}, k_{i}}^{(i)}\right)_{\nu_{i} \in \nabla^{d_{i}}, 1 \leq k_{i} \leq r_{i}}$ with column vectors $\mathbf{U}_{k}^{(i)} \in \ell_{2}\left(\nabla^{d_{i}}\right), k=1, \ldots, r_{i}$, is called the $i$-th mode frame, where we admit $r_{i}=\infty, i=1, \ldots, m$. When writing sometimes for convenience $\left(\mathbf{U}_{k}^{(i)}\right)_{k \in \mathbb{N}}$, although the $\mathbf{U}_{k}^{(i)}$ may be specified through 10 only for $k \leq r_{i}$, it will always be understood to mean $\mathbf{U}_{k}^{(i)}=0$, for $k>r_{i}$.

Note that in a representation of the form (10), by modifying a accordingly, one can always orthogonalize the columns of $\mathbf{U}^{(i)}$ so as to obtain

$$
\left\langle\mathbf{U}_{k}^{(i)}, \mathbf{U}_{l}^{(i)}\right\rangle=\delta_{k l}, \quad i=1, \ldots, m .
$$

We shall refer to $\mathbf{U}^{(i)}$ with the latter property as orthonormal mode frames.

It will be convenient to introduce the following shorthand notation: we denote by $\mathbb{U}$ the system of orthonormal mode frames $\left(\mathbf{U}^{(i)}\right)_{i=1}^{m}$ so that 10 can be rewritten as

$$
\mathbb{U}_{\mathrm{k}}:=\bigotimes_{i=1}^{m} \mathbf{U}_{k_{i}}^{(i)}=\mathbf{U}_{k_{1}}^{(1)} \otimes \cdots \otimes \mathbf{U}_{k_{m}}^{(m)}, \quad \mathrm{k}=\left(k_{1}, \ldots, k_{m}\right) \in \mathbb{N}^{m} .
$$

If $\mathbf{a}$ is represented directly by its entries, (10) corresponds to the so-called Tucker format [33, 34] or subspace representation. The hierarchical Tucker format [20], as well as the special case of the tensor train format [30, correspond to representations in the form (10) as well, but use a further structured representation for the core tensor $\mathbf{a}$.

In this work, for multiindices in $\mathbb{N}_{0}^{t}, t \in \mathbb{N}$, we shall use the notational convention $\mathrm{k}=\left(k_{1}, \ldots, k_{t}\right), \mathrm{n}=\left(n_{1}, \ldots, n_{t}\right), \mathbf{r}=\left(r_{1}, \ldots, r_{t}\right)$, and so forth.

\subsection{Tucker format}

It is instructive to consider first the simpler case of the Tucker format in more detail. 


\subsubsection{Some Prerequisites}

Clearly, any finitely supported $\mathbf{u} \in \ell_{2}\left(\nabla^{d}\right)$ can be represented in the form 10 for some $r=\left(r_{i}\right) \in \mathbb{N}_{0}^{m}$. As mentioned before, for general $\mathbf{u} \in \ell_{2}\left(\nabla^{d}\right)$, the sum in (10) may be infinite. Given a system of mode frames $\mathbb{U}$, representing $\mathbf{u}$ in $(10)$, we define the corresponding rank vector $\operatorname{rank}(\mathbf{u}) \in\left(\mathbb{N}_{0} \cup\{\infty\}\right)^{m}$ by its entries

$$
\operatorname{rank}_{i}(\mathbf{u}):=\operatorname{dim} \operatorname{span}\left\{\mathbf{U}_{k}^{(i)}: k \in \mathbb{N}\right\}, \quad i=1, \ldots, m .
$$

It is not hard to see that this rank vector is independent of the specific mode frame system $\mathbb{U}$. It is referred to as the multilinear rank of $\mathbf{u}$ with respect to $\mathbb{U}$. For such rank vectors $r \in\left(\mathbb{N}_{0} \cup\{\infty\}\right)^{m}$, we introduce the notation

$$
|\mathrm{r}|_{\infty}:=\max _{j=1, \ldots, m} r_{j}
$$

We can then define the set of those sequences $\mathbf{u}$ possessing a representation (10) of a given multilinear rank $r \in\left(\mathbb{N}_{0} \cup\{\infty\}\right)^{m}$ by

$$
\mathcal{T}(\mathrm{r}):=\left\{\mathbf{u} \in \ell_{2}\left(\nabla^{d}\right): \operatorname{rank}_{i}(\mathbf{u}) \leq r_{i}, i=1, \ldots, m\right\} .
$$

The actual computational complexity will eventually depend on the "support" of the $i$-th mode frame given by

$$
\operatorname{supp}_{i}(\mathbf{u}):=\bigcup_{k=1}^{\infty} \operatorname{supp} \mathbf{U}_{k}^{(i)}
$$

Recall that if \# $\operatorname{supp}_{i}(\mathbf{u})<\infty$, necessarily also $\operatorname{rank}_{i}(\mathbf{u})<\infty$.

The following result, which can be found e.g. in [13, 18, 35], ensures the existence of best approximations in $\mathcal{T}(r)$ also for infinite ranks.

Theorem 1. Let $\mathbf{u} \in \ell_{2}\left(\nabla^{d}\right)$ and $0 \leq r_{i} \leq \operatorname{rank}_{i}(\mathbf{u})$, then there exists $\mathbf{v} \in \mathcal{T}(r)$ such that

$$
\|\mathbf{u}-\mathbf{v}\|=\min _{\operatorname{rank}(\mathbf{w}) \leq \mathrm{r}}\|\mathbf{u}-\mathbf{w}\| .
$$

To simplify notation for the sums in 10 , we define for $r \in \mathbb{N}_{0}^{m}$

$$
\mathrm{K}_{m}(\mathrm{r}):= \begin{cases}\times_{i=1}^{m}\left\{1, \ldots, r_{i}\right\} & \text { if } \min \mathrm{r}>0 \\ \emptyset & \text { if } \min \mathrm{r}=0\end{cases}
$$

Moreover, for any vector $\mathrm{x}=\left(x_{i}\right)_{i=1, \ldots, m}$ and for $i \in\{1, \ldots, m\}$, we employ the notation

$$
\begin{aligned}
\check{\mathrm{x}}_{i} & :=\left(x_{1}, \ldots, x_{i-1}, x_{i+1}, \ldots, x_{m}\right), \\
\left.\check{x}_{i}\right|_{y} & :=\left(x_{1}, \ldots, x_{i-1}, y, x_{i+1}, \ldots, x_{m}\right)
\end{aligned}
$$

to refer to the corresponding vector with entry $i$ deleted or entry $i$ replaced by $y$, respectively. We shall also need the auxiliary quantities

$$
a_{p q}^{(i)}:=\sum_{\check{\mathrm{k}}_{i} \in \mathrm{K}_{m-1}\left(\check{\mathrm{r}}_{i}\right)} a_{\left.\check{\mathrm{k}}_{i}\right|_{p}} a_{\left.\check{\mathrm{k}}_{i}\right|_{q}}, \quad \sigma_{p}^{(i)}:=\sqrt{a_{p p}^{(i)}}
$$

derived from the core tensor, where $i \in\{1, \ldots, m\}$ and $p, q \in\left\{1, \ldots, r_{i}\right\}$. 


\subsubsection{Higher-Order Singular Value Decomposition}

With the notions introduced above at hand, one can formulate an analogue of the singular value decomposition of matrices, the higher-order singular value decomposition (HOSVD) [24, for the Tucker tensor format (10). In the following theorem, we summarize its properties in the more general case of infinite-dimensional sequence spaces, where the singular value decomposition is replaced by the spectral theorem for compact operators. These facts could also be extracted from the treatment in [18, Section 8.3].

Theorem 2. For any $\mathbf{u} \in \ell_{2}\left(\nabla^{d}\right)$ there exist orthonormal mode frames $\left\{\mathbf{U}_{k}^{(i)}\right\}_{k \in \mathbb{N}}, i=$ $1, \ldots, m$, with $\mathbf{U}_{k}^{(i)} \in \ell_{2}\left(\nabla^{d_{i}}\right)$ which uniquely determine a core tensor $\mathbf{a}=\left(a_{\mathbf{k}}\right)_{\mathbf{k} \in \mathbb{N}^{m}} \in$ $\ell_{2}\left(\mathbb{N}^{m}\right)$ such that

$$
\mathbf{u}=\sum_{\mathbf{k} \in \mathbb{N}^{m}} a_{\mathrm{k}} \mathbb{U}_{\mathrm{k}}
$$

Moreover, with $a_{p q}^{(i)}$ and $\sigma_{p}^{(i)}$ as in (16), the following hold:

(i) For all $i \in\{1, \ldots, m\}$ we have $\left(\sigma_{k}^{(i)}\right)_{k \in \mathbb{N}} \in \ell_{2}(\mathbb{N})$, and $\sigma_{k}^{(i)} \geq \sigma_{k+1}^{(i)} \geq 0$ for all $k \in \mathbb{N}$.

(ii) For all $i \in\{1, \ldots, m\}$ and all $p, q \in \mathbb{N}$, we have $a_{p q}^{(i)}=\left|\sigma_{p}^{(i)}\right|^{2} \delta_{p q}$.

(iii) For each $\mathrm{r} \in \mathbb{N}_{0}^{m}$, we have

$$
\left\|\mathbf{u}-\sum_{\mathrm{k} \in \mathrm{K}_{m}(\mathrm{r})} a_{\mathrm{k}} \mathbb{U}_{\mathrm{k}}\right\| \leq\left(\sum_{i=1}^{m} \sum_{k=r_{i}+1}^{\infty}\left|\sigma_{k}^{(i)}\right|^{2}\right)^{\frac{1}{2}} \leq \sqrt{m} \inf _{\operatorname{rank}(\mathbf{w}) \leq \mathrm{r}}\|\mathbf{u}-\mathbf{w}\| .
$$

If in addition supp $\mathbf{u} \subseteq \Lambda_{1} \times \cdots \times \Lambda_{m} \subset \nabla^{d}$ for finite $\Lambda_{i} \subset \nabla^{d_{i}}$, then $\operatorname{supp} \mathbf{U}_{k}^{(i)} \subseteq \Lambda_{i}$ and we have supp $\mathbf{a} \subseteq \mathrm{K}_{m}(\overline{\mathrm{r}})$ with $\overline{\mathrm{r}} \in \mathbb{N}_{0}^{m}$ satisfying $\bar{r}_{i} \leq \# \Lambda_{i}$ for $i=1, \ldots, m$.

Proof. Let $\mathbf{u}=\left(u_{\nu}\right)_{\nu \in \nabla^{d}} \in \ell_{2}\left(\nabla^{d}\right)$. For each $i \in\{1, \ldots, m\}$ we consider the mode- $i$ matricization of $\mathbf{u}$, that is, the infinite matrix $\left(u_{\nu, \tilde{\nu}}^{(i)}\right)_{\nu \in \nabla^{d_{i}}, \tilde{\nu} \in \nabla^{d-d_{i}}}$ with entries $u_{\nu_{i}, \check{\nu}_{i}}^{(i)}:=u_{\nu}$ for $\nu \in \nabla^{d}$, which defines a Hilbert-Schmidt operator

$$
T_{\mathbf{u}}^{(i)}: \ell_{2}\left(\nabla^{d-d_{i}}\right) \rightarrow \ell_{2}\left(\nabla^{d_{i}}\right),\left(c_{\tilde{\nu}}\right)_{\tilde{\nu} \in \nabla^{d-d_{i}}} \mapsto\left(\sum_{\tilde{\nu} \in \nabla^{d-d_{i}}} u_{\nu, \tilde{\nu}}^{(i)} c_{\tilde{\nu}}\right)_{\nu \in \nabla^{d_{i}}}
$$

By the spectral theorem, for each $i$ there exist a nonnegative real sequence $\left(\sigma_{n}^{(i)}\right)_{n \in \mathbb{N}}$, where $\sigma_{n}^{(i)}$ are the eigenvalues of $\left(\left(T^{(i)}\right)^{*} T^{(i)}\right)^{1 / 2}$, as well as orthonormal bases $\mathbf{U}^{(i)}=$ $\left\{\mathbf{U}_{n}^{(i)}\right\}_{n \in \mathbb{N}}$ for a subspace of $\ell_{2}\left(\nabla^{d_{i}}\right)$ and $\left\{\mathbf{V}_{n}^{(i)}\right\}_{n \in \mathbb{N}}$ for $\ell_{2}\left(\nabla^{d-d_{i}}\right)$ (again tacitly assuming that $\mathbf{U}_{n}^{(i)}=\mathbf{V}_{n}^{(i)}=0$ for $\left.n>\operatorname{dim} \operatorname{range}\left(T_{\mathbf{u}}^{(i)}\right)\right)$, such that

$$
T_{\mathbf{u}}^{(i)}=\sum_{n \in \mathbb{N}} \sigma_{n}^{(i)}\left\langle\mathbf{V}_{n}^{(i)}, \cdot\right\rangle \mathbf{U}_{n}^{(i)} .
$$

The representation (18) converges in the Hilbert-Schmidt norm, and as a consequence we have

$$
\mathbf{u}=\left(\sum_{n \in \mathbb{N}} \sigma_{n}^{(i)} \mathbf{U}_{\nu_{i}, n}^{(i)} \mathbf{V}_{\check{\nu}_{i}, n}^{(i)}\right)_{\nu \in \nabla^{d}}
$$

with convergence in $\ell_{2}\left(\nabla^{d}\right)$. Furthermore, $\left\{\mathbb{U}_{\mathrm{n}}\right\}_{\mathrm{n} \in \mathbb{N}^{m}}$ with $\mathbb{U}_{\mathrm{n}}:=\bigotimes_{j=1}^{m} \mathbf{U}_{n_{j}}^{(j)}$ is an orthonormal system in $\ell_{2}\left(\nabla^{d}\right)$ (spanning a strict subspace of $\ell_{2}\left(\nabla^{d}\right)$ when $|\operatorname{rank}(\mathbf{u})|_{\infty}<\infty$ ), and setting $a_{\mathrm{n}}:=\left\langle\mathbf{u}, \mathbb{U}_{\mathrm{n}}\right\rangle$, we have $\mathbf{a}=\left(a_{\mathrm{n}}\right) \in \ell_{2}\left(\mathbb{N}^{m}\right)$ and $\mathbf{u}=\sum_{\mathrm{n} \in \mathbb{N}^{m}} a_{\mathrm{n}} \mathbb{U}_{\mathrm{n}}$. The further properties of the expansion can now be obtained along the lines of [24], see also [2, 18]. 
In what follows we shall denote by

$$
\mathbb{U}(\mathbf{u})=\mathbb{U}^{\mathcal{T}}(\mathbf{u}):=\left\{\mathbf{U}^{(i)}: i=1, \ldots, m, \text { generated by HOSVD }\right\}
$$

the particular system of orthonormal mode frames generated for a given $\mathbf{u}$ by HOSVD. It will occasionally be important to identify the specific tensor format to which a given system of mode frames refers, for which we use a corresponding superscript, such as in $\mathbb{U}^{\mathcal{T}}$ for the Tucker format.

The $\sigma_{k}^{(i)}$ are also referred to as mode- $i$ singular values of $\mathbf{u}$. Property (iii) in Theorem 2 leads to a simple procedure for truncation to lower multilinear ranks with an explicit error estimate in terms of the mode- $i$ singular values. In this manner, one does not necessarily obtain the best approximation for prescribed rank, but the approximation is quasi-optimal in the sense that the error is at most by a factor $\sqrt{m}$ larger than the error of best approximation with the same multilinear rank.

We shall make use of the following direct consequence of Theorems 2 .

Corollary 1. Let $\mathbf{u} \in \ell_{2}\left(\nabla^{d}\right)$. For an HOSVD of $\mathbf{u}$ as in Theorem 2, and for $\tilde{\mathbf{r}}$ with $0 \leq \tilde{r}_{i} \leq \operatorname{rank}_{i}(\mathbf{u})$ and

$$
\tilde{\mathbf{u}}=\sum_{\mathrm{k} \in \mathrm{K}_{m}(\tilde{\mathrm{r}})} a_{\mathrm{k}}\left(\bigotimes_{i=1}^{m} \mathbf{U}_{k_{i}}^{(i)}\right),
$$

we have

$$
\|\mathbf{u}-\tilde{\mathbf{u}}\|^{2} \leq \sum_{i=1}^{m} \sum_{k=\tilde{r}_{i}+1}^{\operatorname{rank}_{i}(\mathbf{u})}\left|\sigma_{k}^{(i)}\right|^{2}
$$

and

$$
\|\mathbf{u}-\tilde{\mathbf{u}}\| \leq \sqrt{m} \inf _{\mathbf{w} \in \mathcal{T}(r)}\|\mathbf{u}-\mathbf{w}\| .
$$

Remark 1. Suppose that for a finitely supported vector $\mathbf{u}$ on $\nabla^{d}$, we have a possibly redundant representation

$$
\mathbf{u}=\sum_{\mathrm{k} \in \mathrm{K}_{m}(\tilde{\mathrm{r}})} \tilde{a}_{\mathrm{k}} \bigotimes_{i=1}^{m} \tilde{\mathbf{U}}_{k_{i}}^{(i)},
$$

where the vectors $\tilde{\mathbf{U}}_{k}^{(i)}, k=1, \ldots, \tilde{r}_{i}$ may be linearly dependent. Then by standard linear algebra procedures, we can obtain a HOSVD of $\mathbf{u}$ with a number of arithmetic operations that can be estimated by

$$
C m|\tilde{r}|_{\infty}^{m+1}+C|\tilde{r}|_{\infty}^{2} \sum_{i=1}^{m} \# \operatorname{supp}_{i}(\mathbf{u})
$$

where $C>0$ is an absolute constant (see, e.g., [18]).

\subsubsection{Projections}

We conclude this subsection with introducing canonical projections associated with any given system of orthonormal mode frames, since in what follows they serve as a major work horse. For any system of orthonormal mode frames $\mathbb{V}=\left(\mathbf{V}^{(i)}\right)_{i=1}^{m}$ with $r_{i}$ columns (where $r_{i}$ could be infinity), we consider the $\mathbb{V}$-rigid Tucker class

$$
\mathcal{T}(\mathbb{V}, r):=\left\{\mathrm{P}_{\mathbb{V}} \mathbf{v}: \mathbf{v} \in \ell_{2}\left(\nabla^{d}\right)\right\}
$$


where

$$
\mathrm{P}_{\mathbb{V} \mathbf{v}}:=\sum_{\mathbf{k} \in \mathrm{K}_{m}(\mathrm{r})}\left\langle\bigotimes_{i=1}^{m} \mathbf{V}_{k_{i}}^{(i)}, \mathbf{v}\right\rangle\left(\bigotimes_{i=1}^{m} \mathbf{V}_{k_{i}}^{(i)}\right)
$$

and

$$
\left\langle\mathbf{v}, \bigotimes_{j=1}^{m} \mathbf{V}_{k_{j}}^{(j)}\right\rangle=\sum_{\nu \in \nabla^{d}} v_{\nu} \mathbf{V}_{\nu_{1}, k_{1}}^{(1)} \cdots \mathbf{V}_{\nu_{m}, k_{m}}^{(m)}
$$

Moreover, for any $0 \leq s_{i} \leq r_{i}$, the truncated projection

$$
\mathrm{P}_{\mathbb{V}, \mathbf{s}}=\mathrm{P}_{\mathbb{V}, \mathbf{s}}^{\mathcal{T}}:=\sum_{\mathrm{k} \in \mathrm{K}_{m}(\mathrm{~s})}\left\langle\bigotimes_{i=1}^{m} \mathbf{V}_{k_{i}}^{(i)}, \cdot\right\rangle\left(\bigotimes_{i=1}^{m} \mathbf{V}_{k_{i}}^{(i)}\right),
$$

is a simple mechanism to discard some of the columns producing the best approximation from the reduced spaces $\mathcal{T}(\mathbb{V}, \mathrm{s})$. We shall sometimes use the superscript $\mathcal{T}$ to indicate the reference to a specific tensor format, here the Tucker format. With these definitions, we can write

$$
\mathrm{P}_{\mathbb{V}, \mathrm{r}} \mathbf{v}=\sum_{\mathrm{k} \in \mathrm{K}_{m}(\mathrm{r})}\left\langle\mathbf{v}, \mathbb{V}_{\mathrm{k}}\right\rangle \mathbb{V}_{\mathrm{k}}:=\sum_{\mathrm{k} \in \mathrm{K}_{m}(\mathrm{r})}\left\langle\mathbf{v}, \bigotimes_{j=1}^{m} \mathbf{V}_{k_{j}}^{(j)}\right\rangle \bigotimes_{j=1}^{m} \mathbf{V}_{k_{j}}^{(j)}
$$

Note that at this point we do not require the $\mathbf{V}_{l}^{(i)}$ to have finite support.

Corollary 2. For $\mathbf{u} \in \ell_{2}\left(\nabla^{d}\right)$ and $\mathbf{r}=\left(r_{i}\right)_{i=1}^{m} \in \mathbb{N}_{0}^{m}$ with $0 \leq r_{i} \leq \operatorname{rank}_{i}(\mathbf{u}), i=1, \ldots, m$, there exists an orthonormal mode frame system $\overline{\mathbb{U}}(\mathbf{u}, r)$ such that

$$
\left\|\mathbf{u}-\mathrm{P}_{\overline{\mathbb{U}}(\mathbf{u}, r)} \mathbf{u}\right\|=\min _{\mathbf{w} \in \mathcal{T}(r)}\|\mathbf{u}-\mathbf{w}\|,
$$

with $\mathrm{P}_{\overline{\mathbb{U}}(\mathbf{u}, \mathbf{r})}$ given by 25 .

Proof. By Theorem 1, a best approximation of ranks $r$ for $\mathbf{u}$,

$$
\overline{\mathbf{u}} \in \arg \min \left\{\|\mathbf{u}-\mathbf{v}\|: \operatorname{rank}_{\alpha}(\mathbf{u}) \leq r_{\alpha}\right\},
$$

exists. Defining $\overline{\mathbb{U}}(\mathbf{u}, r):=\mathbb{U}(\overline{\mathbf{u}})$ as the orthonormal mode frame system for $\overline{\mathbf{u}}$, given by the HOSVD, we obtain the assertion.

\subsection{The Hierarchical Tucker Format}

The Tucker format as it stands, in general, still gives rise to an increase of degrees of freedom that is exponential in $d$. One way to mitigate the curse of dimensionality is to further decompose the core tensor a in (10). We now briefly formulate the relevant notions concerning the hierarchical Tucker Format in the present sequence space context, following essentially the developments in 15,20, see also [18.

\subsubsection{Dimension Trees}

Definition 1. Let $m \in \mathbb{N}, m \geq 2$. A set $\mathcal{D}_{m} \subset 2^{\{1, \ldots, m\}}$ is called a dimension tree if the following hold:

(i) $\{1, \ldots, m\} \in \mathcal{D}_{m}$ and for each $i \in\{1, \ldots, m\}$, we have $\{i\} \in \mathcal{D}_{m}$. 
(ii) Each $\alpha \in \mathcal{D}_{m}$ is either a singleton or there exist unique disjoint $\alpha_{1}, \alpha_{2} \in \mathcal{D}_{m}$, called children of $\alpha$, such that $\alpha=\alpha_{1} \cup \alpha_{2}$.

Singletons $\{i\} \in \mathcal{D}_{m}$ are referred to as leaves,

$$
0_{m}:=\{1, \ldots, m\}
$$

as root, and elements of $\mathcal{I}\left(\mathcal{D}_{m}\right):=\mathcal{D}_{m} \backslash\left\{0_{m},\{1\}, \ldots,\{m\}\right\}$ as interior nodes. The set of leaves is denoted by $\mathcal{L}\left(\mathcal{D}_{m}\right)$, where we additionally set $\mathcal{N}\left(\mathcal{D}_{m}\right):=\mathcal{D}_{m} \backslash \mathcal{L}\left(\mathcal{D}_{m}\right)=$ $\mathcal{I}\left(\mathcal{D}_{m}\right) \cup\left\{0_{m}\right\}$. When an enumeration of $\mathcal{L}\left(\mathcal{D}_{m}\right)$ is required, we shall always assume the ascending order with respect to the indices, i.e., in the form $\{\{1\}, \ldots,\{m\}\}$.

It will be convenient to introduce the two functions

$$
\mathrm{c}_{i}: \mathcal{D}_{m} \backslash \mathcal{L}\left(\mathcal{D}_{m}\right) \rightarrow \mathcal{D}_{m} \backslash\left\{0_{m}\right\}, \quad \mathrm{c}_{i}(\alpha):=\alpha_{i}, \quad i=1,2,
$$

producing the "left" and "right" children of a non-leaf node $\alpha$ which, in view of Definition 1. are well-defined up to their order, which we fix by the condition $\min \alpha_{1}<\min \alpha_{2}$.

Note that for a binary dimension tree as defined above, $\# \mathcal{D}_{m}=2 m-1$ and $\# \mathcal{N}\left(\mathcal{D}_{m}\right)=$ $m-1$.

Remark 2. The restriction to binary trees in Definition 1 is not necessary, but leads to the most favorable complexity estimates for algorithms operating on the resulting tensor format. With this restriction dropped, the Tucker format (10) can be treated in the same framework, with the m-ary dimension tree consisting only of root and leaves, i.e., $\left\{0_{m},\{1\}, \ldots,\{m\}\right\}$.

Definition 2. We shall refer to a family

$$
\mathbb{U}=\left\{\mathbf{U}_{k}^{(\alpha)} \in \ell_{2}\left(\nabla^{\sum_{j \in \alpha} d_{j}}\right): \alpha \in \mathcal{D}_{m} \backslash\left\{0_{m}\right\}, k=1, \ldots, k_{\alpha}\right\},
$$

with $k_{\alpha} \in \mathbb{N} \cup\{\infty\}$ for each $\alpha \in \mathcal{D}_{m} \backslash\left\{0_{m}\right\}$, as hierarchical mode frames. In addition, these are called orthonormal if for all $\alpha \in \mathcal{D}_{m} \backslash\left\{0_{m}\right\}$, we have $\left\langle\mathbf{U}_{i}^{(\alpha)}, \mathbf{U}_{j}^{(\alpha)}\right\rangle=\delta_{i j}$ for $i, j=1, \ldots, k_{\alpha}$, and nested if

$$
\begin{aligned}
\overline{\operatorname{span}} & \left\{\mathbf{U}_{k}^{(\alpha)}: k=1, \ldots, k_{\alpha}\right\} \\
& \subseteq \operatorname{span}\left\{\mathbf{U}_{k}^{\left(\mathrm{c}_{1}(\alpha)\right)}: k=1, \ldots, k_{\mathrm{c}_{1}(\alpha)}\right\} \otimes \overline{\operatorname{span}}\left\{\mathbf{U}_{k}^{\left(\mathrm{c}_{2}(\alpha)\right)}: k=1, \ldots, k_{\mathrm{c}_{2} \alpha}\right\} .
\end{aligned}
$$

As for the Tucker format, we set $\mathbf{U}^{(i)}:=\mathbf{U}^{(\{i\})}$, and for $\mathrm{k} \in \mathbb{N}^{m}$ we write

$$
\mathbb{U}_{\mathrm{k}}:=\bigotimes_{i=1}^{m} \mathbf{U}_{k_{i}}^{(i)}
$$
$\mathbb{U}^{\mathcal{H}}$.

Again to express that $\mathbb{U}$ is associated with the hierarchical format we sometimes write

Let $\mathcal{D}_{m}$ be a dimension tree, let $\alpha \in \mathcal{I}\left(\mathcal{D}_{m}\right)$ be an interior node, and $\beta:=\{1, \ldots, m\} \backslash \alpha$. For $\mathbf{u} \in \ell_{2}\left(\nabla^{d}\right)$, we define the Hilbert-Schmidt operator

$$
T_{\mathbf{u}}^{(\alpha)}: \ell_{2}\left(\nabla^{\sum_{i \in \beta} d_{i}}\right) \rightarrow \ell_{2}\left(\nabla^{\sum_{i \in \alpha} d_{i}}\right), \mathbf{c} \mapsto\left(\sum_{\left(\nu_{i}\right)_{i \in \beta}} u_{\nu} c_{\left(\nu_{i}\right)_{i \in \beta}}\right)_{\left(\nu_{i}\right)_{i \in \alpha}},
$$

and set

$$
\operatorname{rank}_{\alpha}(\mathbf{u}):=\operatorname{dim} \operatorname{range} T_{\mathbf{u}}^{(\alpha)}, \quad \alpha \in \mathcal{D}_{m} \backslash 0_{m} .
$$


To be consistent with our previous notation for leaf nodes $\{i\} \in \mathcal{D}_{m}$, we use the abbreviation $\operatorname{rank}_{i}(\mathbf{u}):=\operatorname{rank}_{\{i\}}(\mathbf{u})$. Again, $\operatorname{rank}_{\alpha}(\mathbf{u})$ can be infinite. The root element of the dimension tree, $0_{m}=\{1, \ldots, m\} \in \mathcal{D}_{m}$, is a special case. Here we define

$$
T_{\mathbf{u}}^{\left(0_{m}\right)}: \mathbb{R} \rightarrow \ell_{2}\left(\nabla^{d}\right), t \mapsto t \mathbf{u}
$$

and correspondingly set

$$
\operatorname{rank}_{0_{m}}:=1, \quad \mathbf{U}_{1}^{\left(0_{m}\right)}:=\mathbf{u}, \quad \mathbf{U}_{k}^{\left(0_{m}\right)}:=0, k>1 .
$$

To be consistent with the Tucker format we denote by

$$
\operatorname{rank}(\mathbf{u}):=\left(\operatorname{rank}_{\alpha}(\mathbf{u})\right)_{\alpha \in \mathcal{D}_{m} \backslash\left\{0_{m}\right\}}
$$

the hierarchical rank vector associated with $\mathbf{u}$.

In addition, for the following discussion let $\left\{\mathbf{U}_{k}^{(\alpha)}\right\}_{k=1}^{\operatorname{rank}_{\alpha}(\mathbf{u})}, \mathbf{U}_{k}^{(\alpha)} \in \ell_{2}\left(\nabla^{\sum_{i \in \alpha} d_{i}}\right)$, be the left singular vectors and $\sigma_{k}^{(\alpha)}$ be the singular values of $T_{\mathbf{u}}^{(\alpha)}$. In analogy to the Tucker format we denote by

$$
\left.\mathbb{U}(\mathbf{u})=\mathbb{U}^{\mathcal{H}}(\mathbf{u}):=\left\{\left\{\mathbf{U}_{k}^{(\alpha)}\right\}_{k=1}^{\operatorname{rank}_{\alpha}(\mathbf{u})}: \alpha \in \mathcal{D}_{m} \text {, defined by } 27\right)\right\}
$$

the system of orthonormal hierarchical mode frames with rank vectors $\operatorname{rank}(\mathbf{u})$.

This in turn allows us to define, in analogy to (13), the set of tensors in hierarchical format with ranks $r=\left(r_{\alpha}\right)_{\alpha \in \mathcal{D}_{m} \backslash\left\{0_{m}\right\}}$ as

$$
\mathcal{H}(\mathbf{r}):=\left\{\mathbf{u} \in \ell_{2}\left(\nabla^{d}\right): \operatorname{rank}_{\alpha}(\mathbf{u}) \leq r_{\alpha} \text { for all } \alpha \in \mathcal{D}_{m} \backslash\left\{0_{m}\right\}\right\} .
$$

The observation that the specific systems of hierarchical mode frames $\mathbb{U}(\mathbf{u})$ have the following nestedness property, including the root element, will be crucial.

Proposition 1. For $\mathbf{u} \in \ell_{2}\left(\nabla^{d}\right)$ and $\alpha \in \mathcal{N}\left(\mathcal{D}_{m}\right)$, the mode frames $\left\{\mathbf{U}_{k}^{(\alpha)}\right\}$ given by the left singular vectors of the operators $T_{\mathbf{u}}^{(\alpha)}$ defined in (27) satisfy

$$
\begin{aligned}
\overline{\operatorname{span}}\left\{\mathbf{U}_{k}^{(\alpha)}: k=1, \ldots, \operatorname{rank}_{\alpha}(\mathbf{u})\right\} \subseteq \overline{\operatorname{span}}\left\{\mathbf{U}_{k}^{\left(\mathrm{c}_{1}(\alpha)\right)}: k=1, \ldots, \operatorname{rank}_{\mathrm{c}_{1}(\alpha)}(\mathbf{u})\right\} \\
\otimes \overline{\operatorname{span}}\left\{\mathbf{U}_{k}^{\left(\mathrm{c}_{2}(\alpha)\right)}: k=1, \ldots, \operatorname{rank}_{\mathrm{c}_{2}(\alpha)}(\mathbf{u})\right\},
\end{aligned}
$$

i.e., the family of left singular vectors of the operators $T_{\mathbf{u}}^{(\alpha)}$ is comprized of orthonormal and nested mode frames for $\mathbf{u}$.

Proof. The statement is shown in the more generally applicable framework of minimal subspaces in [18] (cf. Corollary 6.18 and Theorem 6.31 there). For completeness, we give a self-contained proof for our particular setting. Note that, by the properties of the singular value decomposition, we have

$$
\overline{\operatorname{range}} T_{\mathbf{u}}^{(\alpha)}=\overline{\operatorname{span}}\left\{\mathbf{U}_{k}^{(\alpha)}: k=1, \ldots, \operatorname{rank}_{\alpha}(\mathbf{u})\right\}
$$

for all $\alpha \in \mathcal{D}_{m}$. We now fix an $\alpha \in \mathcal{N}\left(\mathcal{D}_{m}\right)$. By construction of the mode frame $\mathbf{U}^{(\alpha)}$, we have the singular value decomposition

$$
\mathbf{u}=\sum_{k=1}^{\operatorname{rank}_{\alpha}(\mathbf{u})} \sigma_{k}^{(\alpha)} \mathbf{U}_{k}^{(\alpha)} \otimes \mathbf{V}_{k}^{(\alpha)}
$$


Furthermore, for each $k$ we may perform a further singular value decomposition to obtain

$$
\mathbf{U}_{k}^{(\alpha)}=\sum_{\ell=1}^{\infty} \hat{\sigma}_{\ell} \mathbf{W}_{k, \ell}^{(1)} \otimes \mathbf{W}_{k, \ell}^{(2)}, \quad \mathbf{W}_{k, \ell}^{(j)} \in \ell_{2}\left(\nabla^{\sum_{i \in c_{j}(\alpha)} d_{i}}\right), j=1,2,
$$

where $\left\|\left(\hat{\sigma}_{\ell}\right)\right\|=1$, and the quantities on the right hand side still depend on $\alpha$. This yields the representation

$$
\mathbf{u}=\sum_{k, \ell} \hat{\sigma}_{\ell} \sigma_{k}^{(\alpha)} \mathbf{W}_{k, \ell}^{(1)} \otimes \mathbf{W}_{k, \ell}^{(2)} \otimes \mathbf{V}_{k}^{(\alpha)}
$$

By orthonormality of the involved basis vectors we have

$$
\begin{aligned}
& T_{\mathbf{u}}^{\left(\mathrm{c}_{1}(\alpha)\right)}\left[\mathbf{W}_{k, \ell}^{(2)} \otimes \mathbf{V}_{k}^{(\alpha)}\right]=\hat{\sigma}_{\ell} \sigma_{k}^{(\alpha)} \mathbf{W}_{k, \ell}^{(1)}, \\
& T_{\mathbf{u}}^{\left(\mathrm{c}_{2}(\alpha)\right)}\left[\mathbf{W}_{k, \ell}^{(1)} \otimes \mathbf{V}_{k}^{(\alpha)}\right]=\hat{\sigma}_{\ell} \sigma_{k}^{(\alpha)} \mathbf{W}_{k, \ell}^{(2)},
\end{aligned}
$$

and hence

$$
\mathbf{W}_{k, \ell}^{(i)} \in \overline{\operatorname{range}} T_{\mathbf{u}}^{\left(\mathrm{c}_{i}(\alpha)\right)}=\overline{\operatorname{span}}\left\{\mathbf{U}_{k}^{\left(\mathrm{c}_{i}(\alpha)\right)}: k=1, \ldots, \operatorname{rank}_{\mathrm{c}_{i}(\alpha)}(\mathbf{u})\right\}
$$

for $i=1,2$. Consequently, any element of $\overline{\operatorname{span}}\left\{\mathbf{U}_{k}^{(\alpha)}: k=1, \ldots, \operatorname{rank}_{\alpha}(\mathbf{u})\right\}$ can be expanded in $\left\{\mathbf{W}_{k, \ell}^{(1)} \otimes \mathbf{W}_{k, \ell}^{(2)}\right\}_{k, \ell}$ and thus also in $\left\{\mathbf{U}_{k_{1}}^{\left(\mathrm{c}_{1}(\alpha)\right)} \otimes \mathbf{U}_{k_{2}}^{\left(\mathrm{c}_{2}(\alpha)\right)}\right\}_{k_{1}, k_{2}}$.

By orthonormality and nestedness, we obtain for each $\alpha \in \mathcal{N}\left(\mathcal{D}_{m}\right)$ and $k=1, \ldots, \operatorname{rank}_{\alpha}(\mathbf{u})$ the expansion

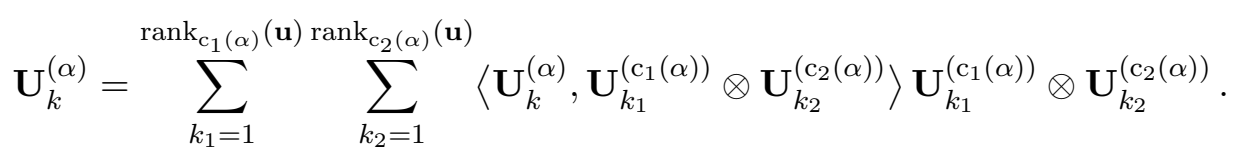

Defining the matrices $\mathbf{B}^{(\alpha, k)} \in \ell_{2}(\mathbb{N} \times \mathbb{N})$ with entries

$$
B_{k_{1}, k_{2}}^{(\alpha, k)}:=\left\langle\mathbf{U}_{k}^{(\alpha)}, \mathbf{U}_{k_{1}}^{\left(\mathrm{c}_{1}(\alpha)\right)} \otimes \mathbf{U}_{k_{2}}^{\left(\mathrm{c}_{2}(\alpha)\right)}\right\rangle
$$

(30) can be rewritten as

$$
\mathbf{U}_{k}^{(\alpha)}=\sum_{k_{1}=1}^{\operatorname{rank}_{\mathrm{c}_{1}(\alpha)}(\mathbf{u})} \sum_{k_{2}=1}^{\operatorname{rank}_{\mathrm{c}_{2}(\alpha)}(\mathbf{u})} B_{k_{1}, k_{2}}^{(\alpha, k)} \mathbf{U}_{k_{1}}^{\left(\mathrm{c}_{1}(\alpha)\right)} \otimes \mathbf{U}_{k_{2}}^{\left(\mathrm{c}_{2}(\alpha)\right)}
$$

providing a decomposition into vectors $\mathbf{U}_{k}^{\mathrm{C}_{i}(\alpha)}, i=1,2$, which now involve shorter multiindices supported in the children $\mathrm{c}_{i}(\alpha)$. This decomposition can be iterated as illustrated by the next step. Abbreviating $\mathrm{c}_{i, j}(\alpha)=\mathrm{c}_{i}\left(\mathrm{c}_{j}(\alpha)\right)$, one obtains

$$
\begin{aligned}
\mathbf{U}_{k}^{(\alpha)}=\sum_{k_{1}=1}^{\operatorname{rank}_{\mathrm{c}_{1}(\alpha)}(\mathbf{u})} \sum_{k_{2}=1}^{\operatorname{rank}_{\mathrm{c}_{2}(\alpha)}(\mathbf{u})} \sum_{\substack{k_{i, 1}, k_{i, 2} k_{j, 1}, k_{j, 2} \\
(i, j) \in\{1,2\}^{2}}} B_{\left(k_{1}, k_{2}\right)}^{(\alpha, k)} \\
\quad \times B_{k_{i, 1}, k_{j, 1}}^{\left(\mathrm{c}_{1}(\alpha), k_{1}\right)} B_{k_{i, 2}, k_{j, 2}}^{\left(\mathrm{c}_{2}(\alpha), k_{2}\right)} \mathbf{U}_{k_{i, 1}}^{\left(\mathrm{c}_{i, 1}(\alpha)\right)} \otimes \mathbf{U}_{k_{j, 1}}^{\left(\mathrm{c}_{j, 1}(\alpha)\right)} \otimes \mathbf{U}_{k_{i, 2}}^{\left(\mathrm{c}_{i, 2}(\alpha)\right)} \otimes \mathbf{U}_{k_{j, 2}}^{\left(\mathrm{c}_{j, 2}(\alpha)\right)} .
\end{aligned}
$$

Applying this recursively, any $\mathbf{u} \in \ell_{2}\left(\nabla^{d}\right)$ can be expanded in the form

$$
\mathbf{u}=\sum_{k_{1}=1}^{\operatorname{rank}_{1}(\mathbf{u})} \cdots \sum_{k_{m}=1}^{\operatorname{rank}_{m}(\mathbf{u})} a_{k_{1}, \ldots, k_{m}} \mathbf{U}_{k_{1}}^{(1)} \otimes \cdots \otimes \mathbf{U}_{k_{m}}^{(m)}
$$


where the core tensor $\mathbf{a}$ has a further decomposition in terms of the matrices $\mathbf{B}^{(\alpha, k)}$ for all non-leaf nodes $\alpha$ and $k=1, \ldots, \operatorname{rank}_{\alpha}(\mathbf{u})$. This decomposition can be given explicitly as follows: For each $\left(k_{\alpha}\right)_{\alpha \in \mathcal{D}_{m}}$, we define the auxiliary expression

$$
\hat{B}_{\left(k_{\alpha}\right)_{\alpha \in \mathcal{D} m}}:=\prod_{\beta \in \mathcal{N}\left(\mathcal{D}_{m}\right)} B_{\left(k_{c_{1}(\beta)}, k_{c_{2}(\beta)}\right)}^{\left(\beta, k_{\beta}\right)} .
$$

We now use this to give an entrywise definition of the tensor $\Sigma_{\mathcal{D}_{m}}\left(\left\{\mathbf{B}^{(\alpha, k)}\right\}\right) \in \ell_{2}\left(\mathbb{N}^{m}\right)$, for each tuple of leaf node indices $\left(k_{\beta}\right)_{\beta \in \mathcal{L}\left(\mathcal{D}_{m}\right)} \in \mathbb{N}^{\# \mathcal{L}\left(\mathcal{D}_{m}\right)}$, as

$$
\left(\Sigma_{\mathcal{D}_{m}}\left(\left\{\mathbf{B}^{(\alpha, k)}: \alpha \in \mathcal{N}\left(\mathcal{D}_{m}\right), k=1, \ldots, \operatorname{rank}_{\alpha}(\mathbf{u})\right\}\right)\right){ }_{\left(k_{\beta}\right)_{\beta \in \mathcal{L}\left(\mathcal{D}_{m}\right)}}=\sum_{\substack{\left(k_{\delta}\right)_{\delta \in \mathcal{I}\left(\mathcal{D}_{m}\right)} \\ k_{\delta}=1, \ldots, \mathrm{rank}_{\delta}(\mathbf{u})}} \hat{B}_{\left(k_{\gamma}\right)_{\gamma \in \mathcal{D}_{m}}} .
$$

Note that the quantity on the right hand side involves a summation over all indices corresponding to non-leaf nodes. Since the summands depend on all indices, this leaves precisely the indices corresponding to leaf nodes as free parameters, as on the left hand side (recall that the index for the root of the tree is restricted to the value 1 ). The tensor defined in (35) then equals the core tensor $\mathbf{a}$, which is thus represented as

$$
\mathbf{a}=\Sigma_{\mathcal{D}_{m}}\left(\left\{\mathbf{B}^{(\alpha, k)}: \alpha \in \mathcal{N}\left(\mathcal{D}_{m}\right), k=1, \ldots, \operatorname{rank}_{\alpha}(\mathbf{u})\right\}\right) .
$$

This representation is illustrated explicitly for $m=4$ in Example 1 below.

Example 1. We consider $m=4$ with $\mathcal{D}_{4}=\{\{1,2,3,4\},\{1,2\},\{3,4\},\{1\},\{2\},\{3\},\{4\}\}$. For this example, we use the abbreviation $r_{\alpha}:=\operatorname{rank}_{\alpha}(\mathbf{u})$ and derive from (33) the expansion

$$
\begin{aligned}
\mathbf{u}=\sum_{k_{1}=1}^{r_{1}} \sum_{k_{2}=1}^{r_{2}} \sum_{k_{3}=1}^{r_{3}} \sum_{k_{4}=1}^{r_{4}} \sum_{k_{\{1,2\}}=1}^{r_{\{1,2\}}} \sum_{k_{\{3,4\}}=1}^{r_{\{3,4\}}} B_{\left(k_{\{1,2\}}, k_{\{3,4\}}\right)}^{(\{1,2,3,4\}, 1)} \\
\times B_{\left(k_{1}, k_{2}\right)}^{\left(\{1,2\}, k_{\{1,2\}}\right)} B_{\left(k_{3}, k_{4}\right)}^{\left(\{3,4\}, k_{\{3,4\}}\right)} \mathbf{U}_{k_{1}}^{(1)} \otimes \mathbf{U}_{k_{2}}^{(2)} \otimes \mathbf{U}_{k_{3}}^{(3)} \otimes \mathbf{U}_{k_{4}}^{(4)},
\end{aligned}
$$

that is, for the core tensor we have the decomposition

$$
a_{k_{1}, k_{2}, k_{3}, k_{4}}=\sum_{k_{\{1,2\}}=1}^{r_{\{1,2\}}} \sum_{k_{\{3,4\}}=1}^{r_{\{3,4\}}} B_{\left(k_{\{1,2\}}, k_{\{3,4\}}\right)}^{(\{1,2,4\}, 1)} B_{\left(k_{1}, k_{2}\right)}^{\left(\{1,2\}, k_{\{1,2\}}\right)} B_{\left(k_{3}, k_{4}\right)}^{\left(\{3,4\}, k_{\{3,4\}}\right)} .
$$

Example 2. A tensor train (TT) representation for $m=4$ as in Example 1 would correspond to $\mathcal{D}_{4}=\{\{1,2,3,4\},\{1\},\{2,3,4\},\{2\},\{3,4\},\{3\},\{4\}\}$, i.e., a degenerate instead of a balanced binary tree. More precisely, the special case of the hierarchical Tucker format resulting from this type of tree has also be considered under the name extended TT format [29].

Following [13, 18], we can formulate now the analogue to Theorem 1 .

Theorem 3. Let $\mathbf{u} \in \ell_{2}\left(\nabla^{d}\right)$, let $\mathcal{D}_{m}$ be a dimension tree, and let $\mathbf{r}=\left(r_{\alpha}\right)$ with $0 \leq r_{\alpha} \leq$ $\operatorname{rank}_{\alpha}(\mathbf{u})$ for $\alpha \in \mathcal{D}_{m} \backslash\left\{0_{m}\right\}$, then there exists $\mathbf{v} \in \mathcal{H}(\mathbf{r})$ such that

$$
\|\mathbf{u}-\mathbf{v}\|=\min \left\{\|\mathbf{u}-\mathbf{w}\|: \operatorname{rank}_{\alpha}(\mathbf{w}) \leq r_{\alpha}, \alpha \in \mathcal{D}_{m} \backslash\left\{0_{m}\right\}\right\} .
$$




\subsubsection{Hierarchical Singular Value Decomposition}

There exists a decomposition for the hierarchical Tucker format that can be regarded as a generalization of the HOSVD, which we shall refer to as hierarchical singular value decomposition or $\mathcal{H S V D}$. The finite-dimensional versions of the following claims have been established in [15]. All arguments given there carry over to the infinite-dimensional case as in the proof of Theorem 2 .

Theorem 4. Let $\mathbf{u} \in \ell_{2}\left(\nabla^{d}\right)$, where $d=d_{1}+\ldots+d_{m}$, and let $\mathcal{D}_{m}$ be a dimension tree. Then $\mathbf{u}$ can be represented in the form

$$
\mathbf{u}=\sum_{\mathbf{k} \in \mathbb{N}^{m}} a_{\mathbf{k}} \mathbb{U}_{\mathbf{k}}, \quad \mathbf{a}=\Sigma_{\mathcal{D}_{m}}\left(\left\{\mathbf{B}^{(\alpha, k)}: \alpha \in \mathcal{N}\left(\mathcal{D}_{m}\right), k=1, \ldots, \operatorname{rank}_{\alpha}(\mathbf{u})\right\}\right)
$$

with $\mathbf{a} \in \ell_{2}\left(\nabla^{d}\right), \mathbf{B}^{(\alpha, k)} \in \ell_{2}(\mathbb{N} \times \mathbb{N})$, for $\alpha \in \mathcal{N}\left(\mathcal{D}_{m}\right), k \in \mathbb{N}$, and where the following hold:

(i) $\left\langle\mathbf{U}_{k}^{(i)}, \mathbf{U}_{l}^{(i)}\right\rangle=\delta_{k l}$ for $i=1, \ldots, m$ and $k, l \in \mathbb{N}$;

(ii) $\operatorname{rank}_{0_{m}}(\mathbf{u})=1,\left\|\mathbf{B}^{\left(0_{m}, 1\right)}\right\|=\|\mathbf{u}\|$, and $\mathbf{B}^{\left(0_{m}, k\right)}=0$ for $k>1$;

(iii) $\left\langle\mathbf{B}^{(\alpha, k)}, \mathbf{B}^{(\alpha, l)}\right\rangle=\delta_{k l}$ for $\alpha \in \mathcal{I}\left(\mathcal{D}_{m}\right)$ and $k, l \in \mathbb{N}$;

(iv) for all $i \in\{1, \ldots, m\}$ we have $\left(\sigma_{k}^{(i)}\right)_{k \in \mathbb{N}} \in \ell_{2}(\mathbb{N})$, and $\sigma_{k}^{(i)} \geq \sigma_{k+1}^{(i)} \geq 0$ for all $k \in \mathbb{N}$;

(v) for all $i \in\{1, \ldots, m\}$ we have $a_{p q}^{(i)}=\left|\sigma_{p}^{(i)}\right|^{2} \delta_{p q}, 1 \leq p, q \leq \operatorname{rank}_{i}(\mathbf{u})$.

We also have the following analogue of Corollary 1, see [15].

Theorem 5. Let $\mathbf{u} \in \ell_{2}\left(\nabla^{d}\right)$. For an $\mathcal{H} S V D$ of $\mathbf{u}$ as in Theorem 4, and defining $\tilde{\mathbf{u}}$ by truncation to hierarchical ranks $\tilde{\mathbf{r}}=\left(\tilde{r}_{\alpha}\right)$, we have

$$
\|\mathbf{u}-\tilde{\mathbf{u}}\|^{2} \leq \sum_{\alpha \in \mathcal{D}_{m}} \sum_{k=\tilde{r}_{\alpha}+1}^{\operatorname{rank}_{\alpha}(\mathbf{u})}\left|\sigma_{k}^{(\alpha)}\right|^{2}
$$

and

$$
\|\tilde{\mathbf{u}}-\mathbf{u}\| \leq \sqrt{2 m-3} \inf \{\|\mathbf{u}-\mathbf{v}\|: \mathbf{v} \in \mathcal{H}(\tilde{\mathbf{r}})\} .
$$

Remark 3. Suppose that, in analogy to Remark 1, a compactly supported vector $\mathbf{u}$ on $\nabla^{d}$ is given in a possibly redundant hierarchical representation

$$
\mathbf{u}=\sum_{\mathrm{k} \in \mathrm{K}_{m}(\tilde{\mathrm{r}})} \tilde{a}_{\mathrm{k}} \bigotimes_{i=1}^{m} \tilde{\mathbf{U}}_{k_{i}}^{(i)}, \quad \tilde{\mathbf{a}}=\Sigma_{\mathcal{D}_{m}}\left(\left\{\tilde{\mathbf{B}}^{\left(\alpha, k_{\alpha}\right)}\right\}\right),
$$

where the summations in the expansion of $\tilde{\mathbf{a}}$ range over $k_{\alpha}=1, \ldots, \tilde{r}_{\alpha}$ for each $\alpha$, and where the vectors $\tilde{\mathbf{U}}_{k}^{(i)}, k=1, \ldots, \tilde{r}_{i}$, and $\tilde{\mathbf{B}}^{(\alpha, k)}, k=1, \ldots, \tilde{r}_{\alpha}$, may be linearly dependent. Employing standard linear algebra procedures, an $\mathcal{H} S V D$ of $\mathbf{u}$ can be computed from such a representation, using a number of operations that can be estimated by

$$
C m\left(\max _{\alpha \in \mathcal{D}_{m} \backslash\left\{0_{m}\right\}} \tilde{r}_{\alpha}\right)^{4}+C\left(\max _{i} \tilde{r}_{i}\right)^{2} \sum_{i=1}^{m} \# \operatorname{supp}_{i}(\mathbf{u}),
$$

where $C>0$ is a fixed constant, cf. [15, Lemma 4.9]. 


\subsubsection{Projections}

In analogy to Section 2.1.3 it will be important to associate suitable orthogonal projections with a given system $\mathbb{V}$ of nested orthonormal mode frames. To this end, $r=\left(r_{\alpha}\right)_{\alpha \in \mathcal{D}_{m} \backslash\left\{0_{m}\right\}}$ stands always for a rank vector for the hierarchical Tucker format. Again $r_{\alpha}=\infty$ is permitted. We begin with introducing an analog to (24), with a slightly more involved definition. The hierarchical $\mathbb{V}$-rigid tensor class of rank $r$ is given by

$$
\mathcal{H}(\mathbb{V}, \mathrm{r}):=\left\{\mathbf{w}: \overline{\operatorname{range}} T_{\mathbf{w}}^{(\alpha)} \subseteq \overline{\operatorname{span}}\left\{\mathbf{V}_{k}^{(\alpha)}: k=1, \ldots, r_{\alpha}\right\}, \alpha \in \mathcal{D}_{m} \backslash\left\{0_{m}\right\}\right\},
$$

where $T_{\mathrm{w}}^{(\alpha)}$ is defined by $(27)$. Clearly $\mathcal{H}(\mathbb{V}, \mathrm{r}) \subset \mathcal{H}(\mathrm{r})$.

In analogy to Section 2.1.3 we address next a truncation of hierarchical ranks to $\tilde{r} \leq r$ for elements in $\mathcal{H}(\mathbb{V}, r)$, when $\mathbb{V}$ is a given system of orthonormal and nested mode frames with ranks $r$. There is, however, a slight complication due to the fact that the resulting mode frames restricted to lower ranks $\tilde{r}$ need no longer be nested. Nevertheless, an approximation with restricted mode frames can still be realized through an operation represented as a sequence of projections involving the given mode frames from $\mathbb{V}$. However, the order in which these projections are applied now matters.

In a way the proof of Lemma 1 already indicates how to proceed, namely restricting first on lower "levels" of the dimension tree. To make this precise we denote by $\mathcal{D}_{m}^{\ell}$ the collection of elements of $\mathcal{D}_{m}$ that have distance exactly $\ell$ to the root (i.e., $\mathcal{D}_{m}^{0}=\left\{0_{m}\right\}$, $\mathcal{D}_{m}^{1}=\left\{\mathrm{c}_{1}\left(0_{m}\right), \mathrm{c}_{2}\left(0_{m}\right)\right\}$ and so forth). Let $L$ be the maximal integer such that $\mathcal{D}_{m}^{L} \neq \emptyset$. For $\ell=1, \ldots, L$, let $\overline{\mathcal{D}}_{m}^{\ell}:=\bigcup\left\{i \in \alpha: \alpha \in \mathcal{D}_{m}^{\ell}\right\}$. Then, given $\mathbb{V}$, and abbreviating

$$
\mathrm{P}_{\mathbb{V}, \alpha, \tilde{r}}:=\sum_{k=1}^{\tilde{r}_{\alpha}}\left\langle\mathbf{V}_{k}^{(\alpha)}, \cdot\right\rangle \mathbf{V}_{k}^{(\alpha)}
$$

we define

$$
P_{\mathbb{V}, \ell, \tilde{r}}:=\left(\bigotimes_{i \in\{1, \ldots, m\} \backslash \overline{\mathcal{D}}_{m}^{\ell}} \mathrm{I}_{i}\right) \otimes\left(\bigotimes_{\alpha \in \mathcal{D}_{m}^{\ell}} \mathrm{P}_{\mathbb{V}, \alpha, \tilde{r}}\right),
$$

with $\mathrm{I}_{i}$ denoting the identity operation on the $i$-th tensor mode. Then, as observed in [15], the truncation operation with mode frames $\mathbb{V}$ restricted to ranks $\tilde{r}$ can be represented as

$$
\mathrm{P}_{\mathbb{V}, \tilde{r}}:=P_{\mathbb{V}, L, \tilde{r}} \cdots P_{\mathbb{V}, 2, \tilde{r}} P_{\mathbb{V}, 1, \tilde{r}}
$$

Here the order is important because the projections $\mathrm{P}_{\mathbb{V}, \alpha, \tilde{r}}, \mathrm{P}_{\mathbb{V}, \beta, \tilde{r}}$ corresponding to $\alpha, \beta \in$ $\mathcal{D}_{m}$ with $\alpha \subset \beta$ do not necessarily commute. Therefore a different order of projections may in fact lead to an end result that has ranks larger than $\tilde{r}$, cf. [15.

Specifically, given $\mathbf{u} \in \ell_{2}\left(\nabla^{d}\right)$, we can choose $\mathbb{V}=\mathbb{U}(\mathbf{u})$ provided by the $\mathcal{H}$ SVD, see (28). Hence $\mathrm{P}_{\mathbb{U}(\mathbf{u}), \tilde{r}} \mathbf{u}$ gives the truncation of $\mathbf{u}$ based on the $\mathcal{H}$ SVD. For this particular truncation an error estimate, in terms of the error of best approximation with rank $\tilde{r}$, is given in Theorem 5 .

Remark 4. By (39), we have a representation of $\tilde{\mathbf{u}}:=\mathrm{P}_{\mathbb{U}(\mathbf{u}), \tilde{r}} \mathbf{u}$ in terms of a sequence of non-commuting orthogonal projections. The result $\tilde{\mathbf{u}}$ may again be represented in terms of the orthonormal and nested mode frames $\tilde{\mathbb{U}}:=\mathbb{U}(\tilde{\mathbf{u}})$.

The situation simplifies if we consider the projection to a fixed nested system of mode frames, without a further truncation of ranks that could entail non-nestedness. 
Lemma 1. Let $\mathbb{V}$ be a family of orthonormal and nested hierarchical mode frames with ranks $\mathrm{r}$. Then there exists a linear projection $\mathrm{P}_{\mathbb{V}}: \ell_{2}\left(\nabla^{d}\right) \rightarrow \mathcal{H}(\mathbb{V}, r)$ such that the unique best approximation in $\mathcal{H}(\mathbb{V}, r)$ of any $\mathbf{u} \in \ell_{2}\left(\nabla^{d}\right)$ is given by $\mathrm{P}_{\mathbb{V}} \mathbf{u}$, that is,

$$
\left\|\mathbf{u}-\mathrm{P}_{\mathbb{V}} \mathbf{u}\right\|=\min _{\mathbf{w} \in \mathcal{H}(\mathbb{V}, \mathbf{r})}\|\mathbf{u}-\mathbf{w}\| .
$$

Proof. The sought projection is given by $\mathrm{P}_{\mathbb{V}}=P_{\mathbb{V}, 1, \mathrm{r}}$, since

$$
P_{\mathbb{V}, L, \mathrm{r}} \cdots P_{\mathbb{V}, 2, \mathrm{r}} P_{\mathbb{V}, 1, \mathrm{r}}=P_{\mathbb{V}, 1, \mathrm{r}}
$$

holds as a consequence of the nestedness property.

Corollary 3. For $\mathbf{u} \in \ell_{2}\left(\nabla^{d}\right)$ and $\mathbf{r}=\left(r_{\alpha}\right)_{\alpha \in \mathcal{D}_{m}}$ with $0 \leq r_{\alpha} \leq \operatorname{rank}_{\alpha}(\mathbf{u})$, there exist orthonormal and nested hierarchical mode frames $\overline{\mathbb{U}}(\mathbf{u}, r)$ such that

$$
\left\|\mathbf{u}-\mathrm{P}_{\overline{\mathbb{U}}(\mathbf{u}, r)} \mathbf{u}\right\|=\min _{\mathbf{w} \in \mathcal{H}(r)}\|\mathbf{u}-\mathbf{w}\|
$$

with $\mathrm{P}_{\overline{\mathbb{U}}(\mathbf{u}, r)}$ as in Lemma 1 .

Proof. By Theorem 3, a best approximation of hierarchical ranks $r$ for $\mathbf{u}$,

$$
\overline{\mathbf{u}} \in \arg \min \left\{\|\mathbf{u}-\mathbf{v}\|: \operatorname{rank}_{\alpha}(\mathbf{u}) \leq r_{\alpha}\right\},
$$

exists. Defining $\overline{\mathbb{U}}(\mathbf{u}, r):=\mathbb{U}(\overline{\mathbf{u}})$ as the nested and orthonormal mode frames for $\overline{\mathbf{u}}$, given by the $\mathcal{H S V D}$, we obtain the assertion with Lemma 1 .

\section{Recompression and Coarsening}

As explained in $\$ 1.2$, iterations of the form $(9)$ provide updates $\mathbf{v}=\mathbf{u}_{k}+\omega\left(\mathbf{f}-\mathbf{A} \mathbf{u}_{k}\right)$ which differ from the unknown $\mathbf{u}$ by some known tolerance. However, even when using a "tensor-friendly" structure of the operator A or a known "tensor-sparsity" of the data $\mathbf{f}$, the arithmetic operations leading to the update $\mathbf{v}$ do not give any clue as to whether the resulting ranks are close to minimal. Hence, one needs a mechanism that realizes a subspace correction leading to tensor representations with ranks at least close to minimal ones. This consists in deriving from the known $\mathbf{v}$ a near best approximation to the unknown $\mathbf{u}$ where the notion of near best in terms of ranks is made precise below. Specifically, suppose that $\mathbf{v} \in \ell_{2}\left(\nabla^{d}\right)$ is an approximation of $\mathbf{u} \in \ell_{2}\left(\nabla^{d}\right)$ which for some $\eta>0$ satisfies

$$
\|\mathbf{u}-\mathbf{v}\|_{\ell_{2}\left(\nabla^{d}\right)} \leq \eta \text {. }
$$

We shall show next how to derive from $\mathbf{v}$ a near-minimal rank tensor approximation to u. Based on our preparations in Sections 2.1.3, 2.2.3, the following developments apply to both formats $\mathcal{F} \in\{\mathcal{T}, \mathcal{H}\}$, in fact to any format $\mathcal{F}$ with associated mode frame systems $\mathbb{U}=\mathbb{U}^{\mathcal{F}}$ (see $(20),(28)$ ) for which one can formulate suitable projections $\mathrm{P}_{\mathbb{V}}^{\mathcal{F}}, \mathrm{P}_{\mathbb{V}, \tilde{r}}^{\mathcal{F}}$ with analogous properties. Accordingly, $\mathcal{R}=\mathcal{R}_{\mathcal{F}}, \mathcal{F} \in\{\mathcal{T}, \mathcal{H}\}$ denotes the respective set of rank vectors $\mathcal{R}_{\mathcal{T}}:=\mathbb{N}_{0}^{m}, \mathcal{R}_{\mathcal{H}}:=\mathcal{D}_{m} \backslash\left\{0_{m}\right\}$. A crucial role in what follows is played by the following immediate consequence of Corollaries 2,3 combined with Theorems 2 , 5.

Remark 5. Let for a given $\mathbf{v} \in \ell_{2}\left(\nabla^{d}\right)$ the mode frame system $\mathbb{U}(\mathbf{v})$ be either $\mathbb{U}^{\mathcal{T}}(\mathbf{v})$ or $\mathbb{U}^{\mathcal{H}}(\mathbf{v})$. Then, for any rank vector $\mathrm{r} \leq \operatorname{rank}(\mathbf{v}), \mathrm{r} \in \mathcal{R}$, one has

$$
\left\|\mathbf{v}-\mathrm{P}_{\mathbb{U}(\mathbf{v}), r} \mathbf{v}\right\| \leq \kappa_{\mathrm{P}}\left\|\mathbf{v}-\mathrm{P}_{\overline{\mathbb{U}}(\mathbf{v}, r)} \mathbf{v}\right\|=\kappa_{\mathrm{P}} \min _{\operatorname{rank}(\mathbf{w}) \leq r}\|\mathbf{u}-\mathbf{w}\|,
$$

where $\kappa_{\mathrm{P}}=\sqrt{m}$ when $\mathcal{F}=\mathcal{T}$, and $\kappa_{\mathrm{P}}=\sqrt{2 m-3}$ when $\mathcal{F}=\mathcal{H}$. 


\subsection{Tensor Recompression}

Given $\mathbf{u} \in \ell_{2}\left(\nabla^{d}\right)$, in what follows by $\mathbb{U}(\mathbf{u})$ we either mean $\mathbb{U}^{\mathcal{T}}(\mathbf{u})$ or $\mathbb{U}^{\mathcal{H}}(\mathbf{u})$, see 20 , (28).

We introduce next two notions of "minimal ranks" $r(\mathbf{u}, \eta), \bar{r}(\mathbf{u}, \eta)$ for a given target accuracy $\eta$, one for the specific mode frame system $\mathbb{U}(\mathbf{u})$ provided by either HOSVD or $\mathcal{H S V D}$, and one for the respective best mode frame systems.

Definition 3. For each $\eta>0$ we choose $\mathrm{r}(\mathbf{u}, \eta) \in \mathcal{R}$ such that

$$
\left\|\mathbf{u}-\mathrm{P}_{\mathbb{U}(\mathbf{u}), \mathrm{r}(\mathbf{u}, \eta)} \mathbf{u}\right\| \leq \eta
$$

with minimal $|\mathrm{r}(\mathbf{u}, \eta)|_{\infty}$, that is,

$$
\mathrm{r}(\mathbf{u}, \eta) \in \arg \min \left\{|\mathbf{r}|_{\infty}: \mathrm{r} \in \mathcal{R},\left\|\mathbf{u}-\mathrm{P}_{\mathbb{U}(\mathbf{u}), \mathrm{r}} \mathbf{u}\right\| \leq \eta\right\} .
$$

Similarly, for each $\eta>0$ we choose $\overline{\mathrm{r}}(\mathbf{u}, \eta) \in \mathcal{R}$ such that

$$
\left\|\mathbf{u}-\mathrm{P}_{\overline{\mathbb{U}}(\mathbf{u}, \overline{\mathrm{r}}(\mathbf{u}, \eta))} \mathbf{u}\right\| \leq \eta,
$$

with minimal $|\overline{\mathrm{r}}(\mathbf{u}, \eta)|_{\infty}$, that is (see Corollary 2 and Remark 5),

$$
\overline{\mathrm{r}}(\mathbf{u}, \eta) \in \arg \min \left\{|\mathbf{r}|_{\infty}: \mathbf{r} \in \mathcal{R}, \exists \mathbf{w} \in \mathcal{F}(r),\|\mathbf{u}-\mathbf{w}\|_{\ell_{2}\left(\nabla^{d}\right)} \leq \eta\right\} .
$$

Recall that the projections $\mathrm{P}_{\mathbb{U}(\mathbf{v}), r}=\mathrm{P}_{\mathbb{U}(\mathbf{v}), r}^{\mathcal{F}}$ to $\mathcal{F}(\mathrm{r})$ are given either by 26) or (39) when $\mathcal{F} \in\{\mathcal{T}, \mathcal{H}\}$, respectively. In both cases, they will be used to define computable coarsening operators for any given $\mathbf{v}$ (of finite support in $\nabla^{d}$ ). In fact, setting

$$
\hat{\mathrm{P}}_{\eta} \mathbf{v}:=\mathrm{P}_{\mathbb{U}(\mathbf{v}), \mathrm{r}(\mathbf{v}, \eta)} \mathbf{v},
$$

we have by definition

$$
\left\|\mathbf{v}-\hat{\mathrm{P}}_{\eta} \mathbf{v}\right\| \leq \eta, \quad\left|\operatorname{rank}\left(\hat{\mathrm{P}}_{\eta} \mathbf{v}\right)\right|_{\infty}=|\mathrm{r}(\mathbf{v}, \eta)|_{\infty} .
$$

Lemma 2. Fix any $\alpha>0$. For any $\mathbf{u}, \mathbf{v}, \eta$ satisfying (40), i.e. $\|\mathbf{u}-\mathbf{v}\| \leq \eta$, one has

$$
\left\|\mathbf{u}-\hat{\mathrm{P}}_{\kappa_{\mathrm{P}}(1+\alpha) \eta} \mathbf{v}\right\| \leq\left(1+\kappa_{\mathrm{P}}(1+\alpha)\right) \eta
$$

while

$$
\left|\operatorname{rank}\left(\hat{\mathrm{P}}_{\kappa_{\mathrm{P}}(1+\alpha) \eta} \mathbf{v}\right)\right|_{\infty}=\left|\mathrm{r}\left(\mathbf{v}, \kappa_{\mathrm{P}}(1+\alpha) \eta\right)\right|_{\infty} \leq|\overline{\mathrm{r}}(\mathbf{u}, \alpha \eta)|_{\infty} .
$$

Proof. Bearing Remark 5 in mind, given $\mathbf{u}$, for the projection $\mathrm{P}_{\overline{\mathbb{U}}(\mathbf{u}, \bar{r}(\mathbf{u}, \alpha \eta))}$ one has

$$
\left\|\mathbf{v}-\mathrm{P}_{\overline{\mathbb{U}}(\mathbf{u}, \overline{\mathrm{r}}(\mathbf{u}, \alpha \eta))} \mathbf{v}\right\| \leq\left\|\left(\mathrm{I}-\mathrm{P}_{\overline{\mathbb{U}}(\mathbf{u}, \overline{\mathrm{r}}(\mathbf{u}, \alpha \eta))}\right)(\mathbf{v}-\mathbf{u})\right\|+\left\|\mathbf{u}-\mathrm{P}_{\overline{\mathbb{U}}(\mathbf{u}, \overline{\mathrm{r}}(\mathbf{u}, \alpha \eta))} \mathbf{u}\right\| \leq(1+\alpha) \eta .
$$

On the other hand, we know that for any $r \in \mathcal{R}$,

$$
\left\|\mathbf{v}-\mathrm{P}_{\mathbb{U}(\mathbf{v}), \mathrm{r}} \mathbf{v}\right\| \leq \kappa_{\mathrm{P}} \inf _{\mathbf{w} \in \mathcal{F}(\mathrm{r})}\|\mathbf{v}-\mathbf{w}\|,
$$

so that, by 47), for $r=\bar{r}(\mathbf{u}, \alpha \eta)$ we have

$$
\left\|\mathbf{v}-\mathrm{P}_{\mathbb{U}(\mathbf{v}), \overline{\mathrm{r}}(\mathbf{u}, \alpha \eta)} \mathbf{v}\right\| \leq \kappa_{\mathrm{P}}(1+\alpha) \eta,
$$

which confirms 46). Estimate (45) follows by triangle inequality. 
Thus, appropriately coarsening $\mathbf{v}$ yields an approximation to $\mathbf{u}$ of still the same quality up to a fixed (dimension-dependent) constant, where the rank of this new approximation is bounded by a minimal rank of a best Tucker or hierarchical Tucker approximation to $\mathbf{u}$ for somewhat higher accuracy.

Let us reinterpret this in terms of minimal ranks, i.e., for $r \in \mathcal{R}$, let

$$
\sigma_{\mathbf{r}}(\mathbf{v})=\sigma_{\mathbf{r}, \mathcal{F}}(\mathbf{v}):=\inf \{\|\mathbf{v}-\mathbf{w}\|: \mathbf{w} \in \mathcal{F}(\mathbf{r})\}, \quad \mathcal{F} \in\{\mathcal{T}, \mathcal{H}\}
$$

We now consider corresponding approximation classes.

Definition 4. We call a positive, strictly increasing $\gamma=(\gamma(n))_{n \in \mathbb{N}_{0}}$ with $\gamma(0)=1$ and $\gamma(n) \rightarrow \infty$, as $n \rightarrow \infty$, a growth sequence. For a given growth sequence $\gamma$, we define

$$
\mathcal{A}(\gamma)=\mathcal{A}_{\mathcal{F}}(\gamma):=\left\{\mathbf{v} \in \ell_{2}\left(\nabla^{d}\right): \sup _{\mathbf{r} \in \mathcal{R}} \gamma\left(|\mathbf{r}|_{\infty}\right) \sigma_{\mathbf{r}, \mathcal{F}}(\mathbf{v})=:|\mathbf{v}|_{\mathcal{A}_{\mathcal{F}}(\gamma)}\right\}
$$

and $\|\mathbf{v}\|_{\mathcal{A}_{\mathcal{F}}(\gamma)}:=\|\mathbf{v}\|+|\mathbf{v}|_{\mathcal{A}_{\mathcal{F}}(\gamma)}$. We call the growth sequence $\gamma$ admissible if

$$
\rho_{\gamma}:=\sup _{n \in \mathbb{N}} \gamma(n) / \gamma(n-1)<\infty,
$$

which corresponds to a restriction to at most exponential growth.

In the particular case when $\gamma(n) \sim n^{s}$ for some $s>0,\|\mathbf{v}\|_{\mathcal{A}_{\mathcal{F}}(() \gamma)}:=\|\mathbf{v}\|+|\mathbf{v}|_{\mathcal{A}_{\mathcal{F}}(() \gamma)}$ is a quasi-norm and $\mathcal{A}_{\mathcal{F}}(() \gamma)$ is a linear space.

Theorem 6. Let $\kappa_{\mathrm{P}}$ be as in Remark 2, and let $\alpha>0$. Assume that $\mathbf{u} \in \mathcal{A}_{\mathcal{F}}(\gamma)$ and that $\mathbf{v} \in \ell_{2}\left(\nabla^{d}\right)$ satisfies $\|\mathbf{u}-\mathbf{v}\| \leq \eta$. Then, defining $\mathbf{w}_{\eta}:=\hat{\mathrm{P}}_{\kappa_{\mathrm{P}}(1+\alpha) \eta} \mathbf{v}$, one has

$$
\left|\operatorname{rank}\left(\mathbf{w}_{\eta}\right)\right|_{\infty} \leq \gamma^{-1}\left(\rho_{\gamma}\|\mathbf{u}\|_{\mathcal{A}_{\mathcal{F}}(\gamma)} /(\alpha \eta)\right), \quad\left\|\mathbf{u}-\mathbf{w}_{\eta}\right\| \leq\left(1+\kappa_{\mathrm{P}}(1+\alpha)\right) \eta,
$$

and

$$
\left\|\mathbf{w}_{\eta}\right\|_{\mathcal{A}_{\mathcal{F}}(\gamma)} \leq C\|\mathbf{u}\|_{\mathcal{A}_{\mathcal{F}}(\gamma)}, \quad \eta>0
$$

where $C=\frac{1+\kappa_{\mathrm{P}}(1+\alpha)}{\alpha}+1$.

Proof. The second relation in (48) has already been shown in Remark 2. We also know from (46) that $\left|\operatorname{rank}\left(\mathbf{w}_{\eta}\right)\right|_{\infty} \leq|\mathrm{r}(\mathbf{u}, \alpha \eta)|_{\infty}$. Thus the first relation in (48) is clear if $|\overline{\mathrm{r}}(\mathbf{u}, \alpha \eta)|_{\infty}=0$. Assume that $|\overline{\mathrm{r}}(\mathbf{u}, \alpha \eta)|_{\infty}>1$. Then for any $\mathrm{r}^{\prime}$ with $\left|\mathrm{r}^{\prime}\right|_{\infty}=|\overline{\mathrm{r}}(\mathbf{u}, \alpha \eta)|_{\infty}-1$, by definition of $|\cdot|_{\mathcal{A}_{\mathcal{F}}(\gamma)}$ we have

$$
|\mathbf{u}|_{\mathcal{A}_{\mathcal{F}}(\gamma)} \geq \gamma\left(\left|\mathbf{r}^{\prime}\right|_{\infty}\right) \sigma_{\mathbf{r}^{\prime}, \mathcal{F}}(\mathbf{u}) \geq \gamma\left(\left|\mathbf{r}^{\prime}\right|_{\infty}\right) \alpha \eta \geq \rho_{\gamma}{ }^{-1} \gamma\left(|\bar{r}(\mathbf{u}, \alpha \eta)|_{\infty}\right) \alpha \eta
$$

Also, when $|\bar{r}(\mathbf{u}, \alpha \eta)|_{\infty}=1$, we have

$$
\sigma_{0, \mathcal{T}}(\mathbf{u})=\|\mathbf{u}\|>\alpha \eta=\gamma(0) \alpha \eta \geq \rho_{\gamma}{ }^{-1} \gamma\left(|\overline{\mathrm{r}}(\mathbf{u}, \alpha \eta)|_{\infty}\right) \alpha \eta .
$$

Therefore

$$
|\overline{\mathrm{r}}(\mathbf{u}, \alpha \eta)|_{\infty} \leq \gamma^{-1}\left(\rho_{\gamma}\|\mathbf{u}\|_{\mathcal{A}_{\mathcal{F}}(\gamma)} /(\alpha \eta)\right),
$$

which is the first relation in 48 .

As for the remaining claim, we need to estimate $\gamma\left(|\mathbf{r}|_{\infty}\right) \sigma_{\mathbf{r}, \mathcal{F}}\left(\mathbf{w}_{\eta}\right)$. Whenever $\left.|r|\right|_{\infty} \geq$ $|\overline{\mathrm{r}}(\mathbf{u}, \alpha \eta)|_{\infty}$ we have, by (46), $\sigma_{\mathrm{r}, \mathcal{F}}\left(\mathbf{w}_{\eta}\right)=0$. So we need to consider only $|\mathrm{r}|_{\infty}<|\overline{\mathrm{r}}(\mathbf{u}, \alpha \eta)|_{\infty}$. By (45),

$$
\left\|\mathbf{w}_{\eta}-\mathrm{P}_{\overline{\mathbb{U}}(\mathbf{u}, r)} \mathbf{u}\right\| \leq\left\|\mathbf{w}_{\eta}-\mathbf{u}\right\|+\left\|\mathbf{u}-\mathrm{P}_{\overline{\mathbb{U}}(\mathbf{u}, r)} \mathbf{u}\right\| \leq\left(1+\kappa_{\mathrm{P}}(1+\alpha)\right) \eta+\sigma_{\mathrm{r}, \mathcal{F}}(\mathbf{u}) .
$$


Therefore, we conclude that for $|\mathbf{r}|_{\infty}<|\overline{\mathrm{r}}(\mathbf{u}, \alpha \eta)|_{\infty}$, since $\sigma_{\overline{\mathrm{r}}(\mathbf{u}, \alpha \eta), \mathcal{F}}(\mathbf{u}) \leq \alpha \eta$ while $\sigma_{\mathrm{r}, \mathcal{F}}(\mathbf{u})>$ $\alpha \eta$

$$
\begin{aligned}
\gamma\left(|\mathbf{r}|_{\infty}\right) \sigma_{\mathbf{r}, \mathcal{F}}\left(\mathbf{w}_{\eta}\right) & \leq \gamma\left(|\mathbf{r}|_{\infty}\right) \frac{\left(1+\kappa_{\mathrm{P}}(1+\alpha)\right) \alpha \eta}{\alpha}+\gamma\left(|\mathbf{r}|_{\infty}\right) \sigma_{\mathbf{r}, \mathcal{F}}(\mathbf{u}) \\
& \leq\left(\frac{1+\kappa_{\mathrm{P}}(1+\alpha)}{\alpha}+1\right) \gamma\left(|\mathbf{r}|_{\infty}\right) \sigma_{\mathbf{r}, \mathcal{F}}(\mathbf{u}) \\
& \leq\left(\frac{1+\kappa_{\mathrm{P}}(1+\alpha)}{\alpha}+1\right)|\mathbf{u}|_{\mathcal{A}_{\mathcal{F}}(\gamma)}
\end{aligned}
$$

\subsection{Coarsening of Mode Frames}

We now turn to a second type of operation for reducing the complexity of given coefficient sequences in tensor representation, an operation that coarsens mode frames by discarding basis indices whose contribution is negligible. We shall use the following standard notions for best $N$-term approximations.

Definition 5. For $\hat{d} \in \mathbb{N}$ and $\Lambda \subset \nabla^{\hat{d}}$, we define the restrictions

$$
\mathrm{R}_{\Lambda} \mathbf{v}:=\mathbf{v} \chi_{\Lambda}, \quad \mathbf{v} \in \ell_{2}\left(\nabla^{\hat{d}}\right)
$$

and for each $N \in \mathbb{N}_{0}$, the errors of best $N$-term approximation

$$
\sigma_{N}(\mathbf{v}):=\inf _{\substack{\Lambda \subset \nabla^{\hat{d}} \\ \# \Lambda \leq N}}\left\|\mathbf{v}-\mathrm{R}_{\Lambda} \mathbf{v}\right\|
$$

The compressibility of $\mathbf{v}$ can again be described through approximation classes. For $s>0$, we denote by $\mathcal{A}^{s}\left(\nabla^{\hat{d}}\right)$ the set of $\mathbf{v} \in \ell_{2}\left(\nabla^{\hat{d}}\right)$ such that

$$
\|\mathbf{v}\|_{\mathcal{A}^{s}\left(\nabla^{\hat{d}}\right)}:=\sup _{N \in \mathbb{N}_{0}}(N+1)^{s} \sigma_{N}(\mathbf{v})<\infty
$$

Endowed with this (quasi-)norm, $\mathcal{A}^{s}\left(\nabla^{\hat{d}}\right)$ becomes a (quasi-)Banach space. When no confusion can arise, we shall suppress the index set dependence and write $\mathcal{A}^{s}=\mathcal{A}^{s}\left(\nabla^{\hat{d}}\right)$.

We deliberately restrict the discussion to polynomial decay rates here since this corresponds to finite Sobolev or Besov regularity. However, with appropriate modifications, the subsequent considerations can be adapted also to approximation classes corresponding to more general growth sequences.

\subsubsection{Tensor Contractions}

Searching through a sequence $\mathbf{u} \in \ell_{2}\left(\nabla^{d}\right)$ (of finite support) would suffer from the curse of dimensionality. Being content with near best $N$-term approximations one can get around this by introducing, for each given $\mathbf{u} \in \ell_{2}\left(\nabla^{d}\right)$, the following quantities formed from contractions of the tensor $\mathbf{u} \otimes \mathbf{u}$.

Definition 6. Let $\mathbf{u} \in \ell_{2}\left(\nabla^{d}\right)$. For $i \in\{1, \ldots, m\}$ we define, using the notation 15,

$$
\pi^{(i)}(\mathbf{u})=\left(\pi_{\nu_{i}}^{(i)}(\mathbf{u})\right)_{\nu_{i} \in \nabla^{d_{i}}}:=\left(\left(\sum_{\check{\nu}_{i}}\left|u_{\nu}\right|^{2}\right)^{\frac{1}{2}}\right)_{\nu_{i} \in \nabla^{d_{i}}} .
$$


With a slight abuse of terminology, we shall refer to these $\pi^{(i)}(\cdot)$ simply as contractions. Their direct computation would involve high-dimensional summations over the index sets $\nabla^{d-d_{i}}$. However, the following observations show how this can be avoided. This makes essential use of the particular orthogonality properties of the tensor formats.

Proposition 2. Let $\mathbf{u} \in \ell_{2}\left(\nabla^{d}\right)$.

(i) We have $\|\mathbf{u}\|=\left\|\pi^{(i)}(\mathbf{u})\right\|, i=1, \ldots, m$.

(ii) Let $\Lambda^{(i)} \subseteq \nabla^{d_{i}}$, then

$$
\left\|\mathbf{u}-\mathrm{R}_{\Lambda^{(1)} \times \cdots \times \Lambda^{(m)}} \mathbf{u}\right\| \leq\left(\sum_{i=1}^{m} \sum_{\nu \in \nabla^{d_{i}} \backslash \Lambda^{(i)}}\left|\pi_{\nu}^{(i)}(\mathbf{u})\right|^{2}\right)^{\frac{1}{2}} .
$$

(iii) Let in addition $\mathbf{U}^{(i)}$ and $\mathbf{a}$ be mode frames and core tensor, respectively, as in Theorems 2 or 4, and let $\left(\sigma_{k}^{(i)}\right)$ be the corresponding sequences of mode-i singular values. Then

$$
\pi_{\nu}^{(i)}(\mathbf{u})=\left(\sum_{k}\left|\mathbf{U}_{\nu, k}^{(i)}\right|^{2}\left|\sigma_{k}^{(i)}\right|^{2}\right)^{\frac{1}{2}}, \quad \nu \in \nabla^{d_{i}}
$$

Proof. Property (i) is clear, and (iii) is a simple consequence of the orthogonality properties of mode frames and core tensor stated in Theorems 2 and 4 . Abbreviating $\tilde{\mathbf{u}}:=$ $\mathrm{R}_{\Lambda^{(1)} \times \cdots \times \Lambda^{(m)}} \mathbf{u}$, property (ii) follows, in view of (i), from

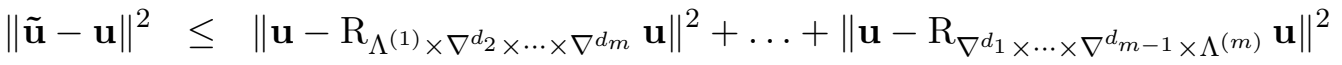

$$
\begin{aligned}
& =\sum_{i=1}^{m} \sum_{\nu \in \nabla^{d_{i}} \backslash \Lambda^{(i)}}\left|\pi_{\nu}^{(i)}(\mathbf{u})\right|^{2} \text {. }
\end{aligned}
$$

The following subadditivity property is an immediate consequence of the triangle inequality.

Proposition 3. Let $N \in \mathbb{N}$ and $\mathbf{u}_{n} \in \ell_{2}\left(\nabla^{d}\right), n=1, \ldots, N$. Then for each $i$ and each $\nu \in \nabla^{d_{i}}$, we have

$$
\pi_{\nu}^{(i)}\left(\sum_{n=1}^{N} \mathbf{u}_{n}\right) \leq \sum_{n=1}^{N} \pi_{\nu}^{(i)}\left(\mathbf{u}_{n}\right) .
$$

Relation (53) allows us to realize (in practice, of course, for finite $\operatorname{ranks} \operatorname{rank}(\mathbf{u})$ and finitely supported mode frames $\mathbf{U}^{(i)}$ ) best $N$-term approximations of the contractions $\pi^{(i)}(\mathbf{u})$ through those of the mode frames $\mathbf{U}_{k}^{(i)}$. Moreover, expressing coarsening errors in terms of tails of contraction sequences requires finding good Cartesian index sets. To see how to determine them let us suppose that for each $i \in\{1, \ldots, m\}$, we have ordered

$$
\pi_{\nu^{i, 1}}^{(i)}(\mathbf{u}) \geq \pi_{\nu^{i, 2}}^{(i)}(\mathbf{u}) \geq \cdots \geq \pi_{\nu^{i, k}}^{(i)}(\mathbf{u}) \geq \cdots .
$$

Then we can also order these quantities jointly for all tensor modes,

$$
\pi_{\tilde{\nu}^{i_{1}, 1}}^{\left(i_{1}\right)}(\mathbf{u}) \geq \pi_{\tilde{\nu}^{i_{2}, 2}}^{\left(i_{2}\right)}(\mathbf{u}) \geq \cdots .
$$

Next, retaining only the $N$ largest from the latter total ordering and redistributing them to the dimension bins

$$
\Lambda^{(i)}(\mathbf{u} ; N):=\left\{\tilde{\nu}^{i_{j}, j}: i_{j}=i, j=1, \ldots, N\right\}, \quad i=1, \ldots, m,
$$


the product set

$$
\Lambda(\mathbf{u} ; N):=\underset{i=1}{m} \Lambda^{(i)}(\mathbf{u} ; N)
$$

can be obtained at a cost that is roughly $m$ times the analogous low-dimensional cost. By construction, one has

$$
\sum_{i=1}^{m} \# \Lambda^{(i)}(\mathbf{u} ; N) \leq N
$$

and

$$
\sum_{i=1}^{m} \sum_{\nu \in \nabla^{d_{i}} \backslash \Lambda^{(i)}(\mathbf{u} ; N)}\left|\pi_{\nu}^{(i)}(\mathbf{u})\right|^{2}=\min _{\hat{\Lambda}}\left\{\sum_{i=1}^{m} \sum_{\nu \in \nabla^{d_{i} \backslash \hat{\Lambda}^{(i)}}}\left|\pi_{\nu}^{(i)}(\mathbf{u})\right|^{2}\right\},
$$

where $\hat{\Lambda}$ ranges over all product sets $\times_{i=1}^{m} \hat{\Lambda}^{(i)}$ with $\sum_{i=1}^{m} \# \hat{\Lambda}^{(i)} \leq N$.

Proposition 4. For any $\mathbf{u} \in \ell_{2}\left(\nabla^{d}\right)$ one has

$$
\left\|\mathbf{u}-R_{\Lambda(\mathbf{u} ; N)} \mathbf{u}\right\|^{2} \leq \sum_{i=1}^{m} \sum_{\nu \in \nabla^{d_{i}} \backslash \Lambda^{(i)}(\mathbf{u} ; N)}\left|\pi_{\nu}^{(i)}(\mathbf{u})\right|^{2},
$$

and for any $\hat{\Lambda}=\times_{i=1}^{m} \hat{\Lambda}^{(i)}$ with $\Lambda^{(i)} \subset \nabla^{d_{i}}$ satisfying $\sum_{i=1}^{m} \# \hat{\Lambda}^{(i)} \leq N$, one has

$$
\left\|\mathbf{u}-R_{\Lambda(\mathbf{u} ; N)} \mathbf{u}\right\| \leq \sqrt{m}\left\|\mathbf{u}-\mathrm{R}_{\hat{\Lambda}} \mathbf{u}\right\| .
$$

Proof. The bound (59) is immediate from $(52)$. Let now $\hat{\Lambda}$ be as in the hypothesis, then by (59) and (58), one obtains

$$
\begin{aligned}
\left\|\mathbf{u}-R_{\Lambda(\mathbf{u} ; N)} \mathbf{u}\right\|^{2} & \leq \sum_{i=1}^{m} \sum_{\nu \in \nabla^{d_{i}} \backslash \hat{\Lambda}^{(i)}}\left|\pi_{\nu}^{(i)}(\mathbf{u})\right|^{2}=\left\|\mathbf{u}-\mathrm{R}_{\hat{\Lambda}^{(1)} \times \nabla^{d_{2} \times \cdots \times \nabla^{d_{m}}}} \mathbf{u}\right\|^{2}+\ldots \\
& +\left\|\mathbf{u}-\mathrm{R}_{\nabla^{d_{1}} \times \cdots \times \nabla^{d_{m-1}} \times \hat{\Lambda}^{(m)}} \mathbf{u}\right\|^{2} \leq m\left\|\mathbf{u}-\mathrm{R}_{\hat{\Lambda}} \mathbf{u}\right\|^{2} .
\end{aligned}
$$

Note that the sorting (54) used in the construction of (56) can be replaced by a quasisorting by binary binning; we shall return to this point in the proof of Remark 9 . With the above preparations at hand we define the coarsening operator

$$
\mathrm{C}_{\mathbf{u}, N} \mathbf{v}:=\mathrm{R}_{\Lambda(\mathbf{u} ; N)} \mathbf{v}, \quad \mathbf{v} \in \ell_{2}\left(\nabla^{d}\right) .
$$

While $\mathrm{C}_{\mathbf{u}, N}$ is computationally feasible, it is not necessarily strictly optimal. However, we remark that or each $N \in \mathbb{N}$, there exists $\bar{\Lambda}(\mathbf{u} ; N)=\times_{i} \bar{\Lambda}^{(i)}(\mathbf{u} ; N)$ such that the best tensor coarsening operator

$$
\overline{\mathrm{C}}_{\mathbf{u}, N} \mathbf{v}:=\mathrm{R}_{\bar{\Lambda}(\mathbf{u} ; N)} \mathbf{v}, \quad \mathbf{v} \in \ell_{2}\left(\nabla^{d}\right),
$$

realizes

$$
\left\|\mathbf{u}-\overline{\mathrm{C}}_{\mathbf{u}, N} \mathbf{u}\right\|=\min _{\sum_{i} \# \operatorname{supp}_{i}(\mathbf{w}) \leq N}\|\mathbf{u}-\mathbf{w}\| .
$$

The next observation is that the contractions are stable under the projections $\mathrm{P}_{\mathbb{U}^{\mathcal{F}}(\mathbf{u}), r}$, $\mathcal{F} \in\{\mathcal{T}, \mathcal{H}\}$. 
Lemma 3. Let $\mathbf{u} \in \ell_{2}\left(\nabla^{d}\right)$. For $i \in\{1, \ldots, m\}, \nu \in \nabla^{d_{i}}$, and any rank vector $\mathbf{r} \in \mathcal{R}$ with $\mathrm{r} \leq \operatorname{rank}(\mathbf{u})$ componentwise, we have

$$
\pi_{\nu}^{(i)}\left(\mathrm{P}_{\mathbb{U}(\mathbf{u}), \mathrm{r}} \mathbf{u}\right) \leq \pi_{\nu}^{(i)}(\mathbf{u}),
$$

where $\mathbb{U}(\mathbf{u})$ either stands for $\mathbb{U}^{\mathcal{T}}(\mathbf{u})$ or $\mathbb{U}^{\mathcal{H}}(\mathbf{u})$ for the Tucker and hierarchical Tucker format, respectively, see (20), (28).

Proof. We consider first the Tucker format. Using the orthnormality of the mode frames $\mathbb{U}(\mathbf{u})$, we obtain

$$
\left(\pi_{\nu}^{(i)}\left(\mathrm{P}_{\mathbb{U}(\mathbf{u}), \mathrm{r}} \mathbf{u}\right)\right)^{2}=\sum_{\check{\mathrm{k}}_{i} \in \mathrm{K}_{m}\left(\check{\mathrm{r}}_{i}\right)}\left(\sum_{k_{i}=1}^{r_{i}} U_{\nu, k_{1}}^{(i)} a_{\mathrm{k}}\right)^{2} .
$$

For any fixed $\check{\mathrm{k}}_{i}$, we have

$$
\sum_{k_{i}=1}^{r_{i}} \sum_{l_{i}=1}^{r_{i}} U_{\nu, k_{i}}^{(i)} U_{\nu, l_{i}}^{(i)} a_{\left.\check{\mathrm{k}}_{i}\right|_{k_{i}}} a_{\left.\breve{\mathrm{k}}_{i}\right|_{l_{i}}}=\left(\sum_{k_{i}=1}^{r_{i}} U_{\nu, k_{i}}^{(i)} a_{\left.\breve{\mathrm{k}}_{i}\right|_{k_{i}}}\right)^{2} \geq 0,
$$

and combining this with (64), with the notation $R:=\operatorname{rank}(\mathbf{u})$, we obtain

$$
\left(\pi_{\nu}^{(i)}\left(\mathrm{P}_{\mathbb{U}(\mathbf{u}), \mathrm{r}} \mathbf{u}\right)\right)^{2} \leq \sum_{k_{i}=1}^{r_{i}} \sum_{l_{i}=1}^{r_{i}} U_{\nu, k_{i}}^{(i)} U_{\nu, l_{i}}^{(i)} \sum_{\check{\mathrm{k}}_{i} \in \mathrm{K}_{m}\left(\check{\mathrm{R}}_{i}\right)} a_{\left.\check{\mathrm{k}}_{i}\right|_{k_{i}}} a_{\left.\check{\mathrm{k}}_{i}\right|_{l_{i}}} .
$$

By Theorem 2(ii), the right hand side equals

$$
\sum_{k_{i}=1}^{r_{i}}\left|\sigma_{k_{i}}^{(i)} U_{\nu, k_{i}}^{(i)}\right|^{2} \leq\left(\pi_{\nu}^{(i)}(\mathbf{u})\right)^{2}
$$

This shows the assertion for the Tucker format. The proof for the hierarchical Tucker format follows similar lines. We treat additional summations arising in the core tensor in the same way as the summation over $\breve{\mathrm{k}}_{i}$ above, and apply the same argument as in (65) recursively.

As a next step, we shall combine the coarsening procedure of this subsection with the tensor recompression considered earlier. To this end, we define $N(\mathbf{v}, \eta):=\min \{N: \| \mathbf{v}-$ $\left.\mathrm{C}_{\mathbf{v}, N} \mathbf{v} \| \leq \eta\right\}$ as well as

$$
\hat{\mathrm{C}}_{\eta}(\mathbf{v}):=\mathrm{C}_{\mathbf{v}, N(\mathbf{v} ; \eta)} \mathbf{v},
$$

in order to switch from (near-)best $N$-term approximation to corresponding (near-)best thresholding procedures. As a consequence of (59), we have

$$
\left\|\mathbf{v}-\mathrm{C}_{\mathbf{v}, N} \mathbf{v}\right\| \leq \kappa_{\mathrm{C}}\left\|\mathbf{v}-\overline{\mathrm{C}}_{\mathbf{v}, N} \mathbf{v}\right\|, \quad \kappa_{\mathrm{C}}=\sqrt{m} .
$$

Our general assumption on the approximability of mode frames is that $\pi^{(i)}(\mathbf{u}) \in \mathcal{A}^{s}$ which, as mentioned before, reflects finite Sobolev or Besov regularity of the lower-dimensional tensor factors. 


\subsubsection{Combination of Tensor Recompression and Coarsening}

Recall that we use $\|\cdot\|_{\mathcal{A}^{s}},\|\cdot\|_{\mathcal{A}_{\mathcal{F}}(\gamma)}$ to quantify sparsity of wavelet expansions of mode frames, and low-rank approximability, respectively. The following main result of this section applies again to both the Tucker and the hierarchical Tucker format. It extends Theorem 6 in combining tensor recompression and wavelet coarsening, and shows that both reduction techniques combined are optimal up to uniform constants, and stable in the respective sparsity norms.

Theorem 7. For a given $\mathbf{v} \in \ell_{2}\left(\nabla^{d}\right)$, let the mode frame system $\mathbb{U}(\mathbf{v})$ be either $\mathbb{U}^{\mathcal{T}}(\mathbf{v})$ or $\mathbb{U}^{\mathcal{H}}(\mathbf{v})$ (see (20), (28)). Let $\mathbf{u}, \mathbf{v} \in \ell_{2}\left(\nabla^{d}\right)$ with $\mathbf{u} \in \mathcal{A}_{\mathcal{F}}(\gamma), \pi^{(i)}(\mathbf{u}) \in \mathcal{A}^{s}$ for $i=1, \ldots, m$, and $\|\mathbf{u}-\mathbf{v}\| \leq \eta$. As before let $\kappa_{\mathrm{P}}=\kappa_{\mathrm{C}}=\sqrt{m}$ for the Tucker format, while for the $\mathcal{H}$-Tucker format $\kappa_{\mathrm{P}}=\sqrt{2 m-3}$ and $\kappa_{\mathrm{C}}=\sqrt{m}$. Then for

$$
\mathbf{w}_{\eta}:=\hat{\mathrm{C}}_{\kappa_{\mathrm{C}}\left(\kappa_{\mathrm{P}}+1\right)(1+\alpha) \eta}\left(\hat{\mathrm{P}}_{\kappa_{\mathrm{P}}(1+\alpha) \eta}(\mathbf{v})\right),
$$

we have

$$
\left\|\mathbf{u}-\mathbf{w}_{\eta}\right\| \leq\left(1+\kappa_{\mathrm{P}}(1+\alpha)+\kappa_{\mathrm{C}}\left(\kappa_{\mathrm{P}}+1\right)(1+\alpha)\right) \eta
$$

as well as

$$
\left|\operatorname{rank}\left(\mathbf{w}_{\eta}\right)\right|_{\infty} \leq \gamma^{-1}\left(\rho_{\gamma}\|\mathbf{u}\|_{\mathcal{A}_{\mathcal{F}}(\gamma)} /(\alpha \eta)\right), \quad\left\|\mathbf{w}_{\eta}\right\|_{\mathcal{A}_{\mathcal{F}}(\gamma)} \leq C_{1}\|\mathbf{u}\|_{\mathcal{A}_{\mathcal{F}}(\gamma)}
$$

with $C_{1}=\left(\alpha^{-1}\left(1+\kappa_{\mathrm{P}}(1+\alpha)\right)+1\right)$ and

$$
\begin{aligned}
& \sum_{i=1}^{m} \# \operatorname{supp}_{i}\left(\mathbf{w}_{\eta}\right) \leq m \eta^{-\frac{1}{s}}\left(\sum_{i=1}^{m}\left\|\pi^{(i)}(\mathbf{u})\right\|_{\mathcal{A}^{s}}\right)^{\frac{1}{s}} \\
& \sum_{i=1}^{m}\left\|\pi^{(i)}\left(\mathbf{w}_{\eta}\right)\right\|_{\mathcal{A}^{s}} \leq C_{2} \sum_{i=1}^{m}\left\|\pi^{(i)}(\mathbf{u})\right\|_{\mathcal{A}^{s}}
\end{aligned}
$$

with $C_{2}=2^{s}\left(1+2^{s}\right)+2^{2 s}\left(\kappa_{\mathrm{P}}(1+\alpha)+\kappa_{\mathrm{C}}\left(\kappa_{\mathrm{P}}(1+\alpha)+1+\alpha\right)\right) m^{\max \{1, s\}}$.

Proof. Taking 45 in Lemma 2 and the definition (66) into account, the relation 69) follows from the triangle inequality.

The statements in 70 follow from Theorem 6 . Note that the additional mode frame coarsening considered here does not affect these estimates.

For the proof of $(69)$ and $(71)$, we can proceed similary to [8, Corollary 5.2] (see also $\left[7\right.$, Theorem 4.9.1]). We set $\hat{\mathbf{w}}:=\hat{\mathrm{P}}_{\kappa_{\mathrm{P}}(1+\alpha) \eta}(\mathbf{v})$. Let $N \in \mathbb{N}$ be minimal such that $\left\|\mathbf{u}-\overline{\mathrm{C}}_{\mathbf{u}, N} \mathbf{u}\right\| \leq \alpha \eta$. Then

$$
\begin{aligned}
\left\|\hat{\mathbf{w}}-\overline{\mathrm{C}}_{\mathbf{u}, N} \hat{\mathbf{w}}\right\| & \leq\left\|\left(\mathrm{I}-\overline{\mathrm{C}}_{\mathbf{u}, N}\right)(\mathbf{u}-\hat{\mathbf{w}})\right\|+\left\|\mathbf{u}-\overline{\mathrm{C}}_{\mathbf{u}, N} \mathbf{u}\right\| \\
& \leq\|\mathbf{u}-\hat{\mathbf{w}}\|+\left\|\mathbf{u}-\overline{\mathrm{C}}_{\mathbf{u}, N} \mathbf{u}\right\| \leq\left(1+\kappa_{\mathrm{P}}(1+\alpha)+\alpha\right) \eta,
\end{aligned}
$$

where we have used Remark 2 to bound the first summand on the right hand side. Consequently, by 67),

$$
\left\|\hat{\mathbf{w}}-\mathrm{C}_{\hat{\mathbf{w}}, N} \hat{\mathbf{w}}\right\| \leq \kappa_{\mathrm{C}}\left\|\hat{\mathbf{w}}-\overline{\mathrm{C}}_{\hat{\mathbf{w}}, N} \hat{\mathbf{w}}\right\| \leq \kappa_{\mathrm{C}}\left\|\hat{\mathbf{w}}-\overline{\mathrm{C}}_{\mathbf{u}, N} \hat{\mathbf{w}}\right\| \leq \kappa_{\mathrm{C}}\left(1+\kappa_{\mathrm{P}}(1+\alpha)+\alpha\right) \eta .
$$

Furthermore, keeping the definition $(62)$ and the optimality $(63)$ in mind, 52 yields

$$
\begin{aligned}
\left\|\mathbf{u}-\overline{\mathrm{C}}_{\mathbf{u}, N} \mathbf{u}\right\| & \leq \inf _{\sum_{i} \# \Lambda_{i} \leq N}\left(\sum_{i=1}^{m}\left\|\pi^{(i)}(\mathbf{u})-\mathrm{R}_{\Lambda_{i}} \pi^{(i)}(\mathbf{u})\right\|^{2}\right)^{\frac{1}{2}} \\
& \leq \sum_{i=1}^{m} \inf _{\# \Lambda_{i} \leq N / m}\left\|\pi^{(i)}(\mathbf{u})-\mathrm{R}_{\Lambda_{i}} \pi^{(i)}(\mathbf{u})\right\| \leq(N / m)^{-s} \sum_{i=1}^{m}\left\|\pi^{(i)}(\mathbf{u})\right\|_{\mathcal{A}^{s}} .
\end{aligned}
$$


Using the latter estimate and noting that the coarsening operator $\hat{\mathrm{C}}_{\kappa_{\mathrm{C}}\left(1+\kappa_{\mathrm{P}}\right)(1+\alpha) \eta}$ retains at most $N$ terms by $(72)$, we conclude that

$$
\sum_{i=1}^{m} \# \operatorname{supp}_{i}\left(\mathbf{w}_{\eta}\right) \leq N \leq \eta^{-\frac{1}{s}} m\left(\sum_{i=1}^{m}\left\|\pi^{(i)}(\mathbf{u})\right\|_{\mathcal{A}^{s}}\right)^{\frac{1}{s}}
$$

and hence the first statement in (71). Resolving the latter inequality for $\eta$, one can rewrite 69 as

$$
\left\|\mathbf{u}-\mathbf{w}_{\eta}\right\| \leq\left(\sum_{i=1}^{m} \# \operatorname{supp}_{i}\left(\mathbf{w}_{\eta}\right)\right)^{-s} C(\alpha) m^{s}\left(\sum_{i=1}^{m}\left\|\pi^{(i)}(\mathbf{u})\right\|_{\mathcal{A}^{s}}\right),
$$

where $C(\alpha):=\kappa_{\mathrm{P}}(1+\alpha)+\kappa_{\mathrm{C}}\left(\kappa_{\mathrm{P}}(1+\alpha)+1+\alpha\right)$. Now let $\hat{N}=\sum_{i=1}^{m} \hat{N}_{i}$ with $\hat{N}_{i}:=$ $\# \operatorname{supp}_{i}\left(\mathbf{w}_{\eta}\right)$. Let $\hat{\mathbf{u}}_{i}$ be the best $\hat{N}_{i}$-term approximation to $\pi^{(i)}(\mathbf{u})$, then

$$
\begin{aligned}
\left\|\pi^{(i)}\left(\mathbf{w}_{\eta}\right)\right\|_{\mathcal{A}^{s}} & \leq 2^{s}\left(\left\|\hat{\mathbf{u}}_{i}\right\|_{\mathcal{A}^{s}}+\left\|\hat{\mathbf{u}}_{i}-\pi^{(i)}\left(\mathbf{w}_{\eta}\right)\right\|_{\mathcal{A}^{s}}\right) \\
& \leq 2^{s}\left(\left\|\pi^{(i)}(\mathbf{u})\right\|_{\mathcal{A}^{s}}+\left(2 \hat{N}_{i}\right)^{s}\left\|\hat{\mathbf{u}}_{i}-\pi^{(i)}\left(\mathbf{w}_{\eta}\right)\right\|\right) \\
& \leq 2^{s}\left(\left\|\pi^{(i)}(\mathbf{u})\right\|_{\mathcal{A}^{s}}+\left(2 \hat{N}_{i}\right)^{s}\left(\left\|\hat{\mathbf{u}}_{i}-\pi^{(i)}(\mathbf{u})\right\|+\left\|\pi^{(i)}(\mathbf{u})-\pi^{(i)}\left(\mathbf{w}_{\eta}\right)\right\|\right)\right) \\
& \left.\leq 2^{s}\left(\left(1+2^{s}\right)\left\|\pi^{(i)}(\mathbf{u})\right\|_{\mathcal{A}^{s}}+\left(2 \hat{N}_{i}\right)^{s}\left\|\pi^{(i)}(\mathbf{u})-\pi^{(i)}\left(\mathbf{w}_{\eta}\right)\right\|\right)\right),
\end{aligned}
$$

where we have used that $\left\|\hat{\mathbf{u}}_{i}-\pi^{(i)}(\mathbf{u})\right\| \leq \hat{N}_{i}^{-s}\left\|\pi^{(i)}(\mathbf{u})\right\|_{\mathcal{A}^{s}}$ as well as \# $\operatorname{supp}_{i}\left(\mathbf{w}_{\eta}\right)$, $\# \operatorname{supp}_{i}\left(\pi^{(i)}\left(\mathbf{w}_{\eta}\right)\right) \leq \hat{N}_{i}$. Moreover, as a consequence of the Cauchy-Schwarz inequality, we have the componentwise estimate

$$
\left|\pi_{\nu}^{(i)}(\mathbf{u})-\pi_{\nu}^{(i)}\left(\mathbf{w}_{\eta}\right)\right| \leq \pi_{\nu}^{(i)}\left(\mathbf{u}-\mathbf{w}_{\eta}\right),
$$

which combined with 73 yields

$$
\begin{aligned}
\left\|\pi^{(i)}(\mathbf{u})-\pi^{(i)}\left(\mathbf{w}_{\eta}\right)\right\| & \leq\left\|\pi^{(i)}\left(\mathbf{u}-\mathbf{w}_{\eta}\right)\right\|=\left\|\mathbf{u}-\mathbf{w}_{\eta}\right\| \\
& \leq \hat{N}^{-s} C(\alpha) m^{s}\left(\sum_{k=1}^{m}\left\|\pi^{(k)}(\mathbf{u})\right\|_{\mathcal{A}^{s}}\right) .
\end{aligned}
$$

Hence we obtain

$$
\left\|\pi^{(i)}\left(\mathbf{w}_{\eta}\right)\right\|_{\mathcal{A}^{s}} \leq 2^{s}\left(1+2^{s}\right)\left\|\pi^{(i)}(\mathbf{u})\right\|_{\mathcal{A}^{s}}+2^{2 s} C(\alpha) m^{s} \hat{N}^{-s} \hat{N}_{i}^{s}\left(\sum_{k=1}^{m}\left\|\pi^{(k)}(\mathbf{u})\right\|_{\mathcal{A}^{s}}\right) .
$$

Summing over $i=1, \ldots, m$ and noting that $\hat{N}^{-s} \sum_{i=1}^{m} \hat{N}_{i}^{s} \leq m^{\max \{0,1-s\}}$, we arrive at the second assertion in (71).

\section{Adaptive Approximation of Operators}

Whether the solution to an operator equation actually exhibits some tensor- and expansion sparsity is expected to depend strongly on the structure of the involved operator. The purpose of this section is formulate a class of operators which are "tensor-friendly" in the sense that their approximate application does not increase the rank too much. Making this precise requires some model assumptions which at this point we feel are relevant in that a wide range of interesting cases is covered. But of course, many possible variants would be conceivable as well. In that sense the main issue in the subsequent discussion 
is to identify the essential structural mechanisms that would still work under somewhat different model assumptions.

We shall approach this on two levels. First we consider operators with an exact low rank structure. Of course, assuming that the operator is a single tensor product of operators acting on functions of a smaller number of variables would be far too restrictive and also concern a trivial scenario, since ranks would be preserved. More interesting are sums of tensor products such as the $m$-dimensional Laplacian

$$
\Delta=\partial_{x_{1}}^{2}+\cdots+\partial_{x_{m}}^{2}
$$

where strictly speaking each summand $\partial_{x_{j}}$ is a tensor product the identity operators acting on all but the $j$ th variable and the second order partial derivative with respect to the $j$ th variable. Hence the wavelet representation $\mathbf{A}$ of $\Delta$ in an $L_{2}$-orthonormal wavelet basis has the form

$$
\mathbf{A}=\mathbf{A}_{1} \otimes \mathrm{I}_{2} \otimes \cdots \otimes \mathrm{I}_{m}+\cdots+\mathrm{I}_{1} \otimes \cdots \otimes \mathrm{I}_{m-1} \otimes \mathbf{A}_{m}
$$

where $\mathbf{A}_{j}$ is the wavelet representation of $\partial_{x_{j}}$. There is, however, an issue concerning the scaling of the wavelet bases. For $L_{2}$-orthonormalized wavelets $\mathbf{A}$ is not bounded, an issue to be taken up later again in Remark 15 .

At a second stage it is important to cover also operators which do not have an explicit low-rank structure but can be approximated in a quantified manner by low-rank operators. A typical example are potential terms, such as those arising in electronic structure calculations, see, e.g., $[2]$ and the references cited there.

\subsection{Operators with Explicit Low-Rank Form}

We start with a technical observation that will be used at several points. Given operators $\mathbf{B}^{(i)}=\left(b_{\nu_{i}, \tilde{\nu}_{i}}^{(i)}\right)_{\nu_{i}, \tilde{\nu}_{i} \in \nabla^{d_{i}}}: \ell_{2}\left(\nabla^{d_{i}}\right) \rightarrow \ell_{2}\left(\nabla^{d_{i}}\right)$, recall that their tensor product $\mathbf{B}=\mathbf{B}^{(1)} \otimes \cdots \otimes$ $\mathbf{B}^{(m)}$ is given by $\mathbf{B}_{\nu, \tilde{\nu}}=b_{\nu_{1}, \tilde{\nu}_{1}}^{(1)} \cdots b_{\nu_{m}, \tilde{\nu}_{m}}^{(m)}$ so that, whenever $\mathbf{v}=\mathbf{v}^{1} \otimes \cdots \otimes \mathbf{v}^{m}, \mathbf{v}^{j} \in \ell_{2}\left(\nabla^{d_{j}}\right)$, we have $\mathbf{B} \mathbf{v}=\left(\mathbf{B}^{(1)} \mathbf{v}^{1}\right) \otimes \cdots \otimes\left(\mathbf{B}^{(m)} \mathbf{v}^{m}\right)$. Observing that for any $\mathbf{v} \in \ell_{2}(\nabla)$

$$
\mathbf{B v}=\left(\mathrm{I}_{1} \otimes \mathbf{B}^{(2)} \otimes \cdots \otimes \mathbf{B}^{(m)}\right)\left(\left(\mathbf{B}^{(1)} \otimes \mathrm{I}_{2} \otimes \cdots \otimes \mathrm{I}_{m}\right) \mathbf{v}\right),
$$

we conclude

$$
\|\mathbf{B v}\| \leq\left\|\mathbf{B}^{(2)} \otimes \cdots \otimes \mathbf{B}^{(m)}\right\|\left\|\pi^{(1)}\left(\left(\mathbf{B}^{(1)} \otimes \mathrm{I}_{2} \otimes \cdots \otimes \mathrm{I}_{m}\right) \mathbf{v}\right)\right\|
$$

More generally, one obtains by the same argument

$$
\begin{aligned}
\|\mathbf{B v}\| \leq \| \mathbf{B}^{(1)} \otimes \cdots \otimes \mathbf{B}^{(i-1)} \otimes & \mathbf{B}^{(i+1)} \otimes \cdots \otimes \mathbf{B}^{(m)} \| \\
& \times\left\|\pi^{(i)}\left(\left(\mathrm{I}_{1} \otimes \mathrm{I}_{i-1} \otimes \mathbf{B}^{(i)} \otimes \mathrm{I}_{i+1} \otimes \cdots \otimes \mathrm{I}_{m}\right) \mathbf{v}\right)\right\| .
\end{aligned}
$$

Similarly, one derives from 75 the inequality

$$
\begin{aligned}
\pi^{(i)}(\mathbf{B v})_{\nu_{i}} \leq\left\|\mathbf{B}^{(1)} \otimes \cdots \otimes \mathbf{B}^{(i-1)} \otimes \mathbf{B}^{(i+1)} \otimes \cdots \otimes \mathbf{B}^{(m)}\right\| & \\
& \times \pi^{(i)}\left(\left(\mathrm{I}_{1} \otimes \cdots \otimes \mathrm{I}_{i-1} \otimes \mathbf{B}^{(i)} \otimes \mathrm{I}_{i+1} \otimes \cdots \otimes \mathrm{I}_{m}\right) \mathbf{v}\right)_{\nu_{i}}, \quad \nu_{i} \in \nabla^{d_{i}} .
\end{aligned}
$$




\subsubsection{Tucker Format}

We shall be concerned first with (wavelet representations of) operators $\mathbf{A}=\left(a_{\nu, \tilde{\nu}}\right)_{\nu, \tilde{\nu} \in \nabla^{d}}$ : $\ell_{2}\left(\nabla^{d}\right) \rightarrow \ell_{2}\left(\nabla^{d}\right)$ composed of tensor products of operators according to the Tucker format. For a given rank vector $\mathrm{R} \in \mathbb{N}^{m}$ throughout this section we assume that $\mathbf{A}: \ell_{2}\left(\nabla^{d}\right) \rightarrow$ $\ell_{2}\left(\nabla^{d}\right)$ is bounded and has the form

$$
\mathbf{A}=\sum_{\mathrm{n} \in \mathrm{K}_{m}(\mathrm{R})} c_{\mathrm{n}} \bigotimes_{i=1}^{m} \mathbf{A}_{n_{i}}^{(i)}
$$

where $\mathbf{A}_{n_{i}}^{(i)}: \ell_{2}\left(\nabla^{d_{i}}\right) \rightarrow \ell_{2}\left(\nabla^{d_{i}}\right)$ for $i \in\{1, \ldots, m\}$ and $n_{i} \in\left\{1, \ldots, R_{i}\right\}$.

Example 3. In particular, any operator of the form

$$
\mathbf{A}_{1} \otimes \mathrm{I}_{2} \otimes \cdots \otimes \mathrm{I}_{m}+\ldots+\mathrm{I}_{1} \otimes \cdots \otimes \mathrm{I}_{m-1} \otimes \mathbf{A}_{m}
$$

can be written in the form $(78)$ with $\mathrm{R}=(2, \ldots, 2), \mathbf{A}_{1}^{(i)}=\mathrm{I}_{i}, \mathbf{A}_{2}^{(i)}=\mathbf{A}_{i}$ for $i=1, \ldots, m$, and core tensor

$$
c_{2,1, \ldots, 1}=\ldots=c_{1, \ldots, 1,2,1, \ldots, 1}=\ldots=c_{1, \ldots, 1,2}=1, \quad c_{\mathrm{n}}=0 \text { otherwise. }
$$

The $\mathbf{A}_{n_{i}}^{(i)}$ are in general infinite matrices and not necessarily sparse in the strict sense. We shall further require, however, that they are nearly sparse as will be quantified next. To this end, suppose that for each $\mathbf{A}_{n_{i}}^{(i)}$ we have a sequence of approximations (in the spectral norm) such that for a given sequence $\varepsilon_{n_{i}, p}^{(i)}, p \in \mathbb{N}$, of tolerances

$$
\left\|\mathbf{A}_{n_{i}}^{(i)}-\tilde{\mathbf{A}}_{n_{i},[p]}^{(i)}\right\| \leq \varepsilon_{n_{i}, p}^{(i)}, \quad p \in \mathbb{N}_{0} .
$$

Moreover, it will be important to apply such sparsified versions of the $\mathbf{A}_{n_{i}}^{(i)}$ to vectors which are supported on the elements of a partition $\left\{\Lambda_{n_{i},[p]}^{(i)}\right\}_{p \in \mathbb{N}_{0}}$ of $\nabla^{d_{i}}$. We shall then consider approximations $\tilde{\mathbf{A}}$ to $\mathbf{A}$ of the form

$$
\tilde{\mathbf{A}}=\sum_{\mathbf{n} \in \mathrm{K}_{m}(\mathrm{R})} c_{\mathrm{n}} \bigotimes_{i=1}^{m} \tilde{\mathbf{A}}_{n_{i}}^{(i)}, \quad \tilde{\mathbf{A}}_{n_{i}}^{(i)}:=\sum_{p \in \mathbb{N}_{0}} \tilde{\mathbf{A}}_{n_{i},[p]}^{(i)} \mathrm{R}_{\Lambda_{n_{i},[p]}^{(i)}},
$$

where as before $R_{\Lambda}$ denotes the restriction of a given input sequence to $\Lambda$. The following lemma describes the accuracy of such approximations.

Lemma 4. Let $\mathbf{v} \in \ell_{2}\left(\nabla^{d}\right)$, let $\mathbf{A}: \ell_{2}\left(\nabla^{d}\right) \rightarrow \ell_{2}\left(\nabla^{d}\right)$ have the form $(78)$ for some $\mathrm{R} \in \mathbb{N}^{m}$, while $\tilde{\mathbf{A}}$, given by 80 satisfies 790 . Then we have

$$
\|\mathbf{A} \mathbf{v}-\tilde{\mathbf{A}} \mathbf{v}\| \leq \sum_{i=1}^{m} \sum_{n_{i}=1}^{R_{i}} \sum_{p \in \mathbb{N}_{0}} C_{\tilde{\mathbf{A}}}^{(i)} \varepsilon_{n_{i},[p]}^{(i)}\left\|\mathrm{R}_{\Lambda_{n_{i},[p]}^{(i)}} \pi^{(i)}(\mathbf{v})\right\|,
$$

where

$$
C_{\tilde{\mathbf{A}}}^{(i)}=\max _{n_{i}=1, \ldots, R_{i}}\left\|\sum_{\check{n}_{i}} c_{\mathrm{n}}\left(\bigotimes_{j=1}^{i-1} \tilde{\mathbf{A}}_{n_{j}}^{(j)}\right) \otimes\left(\bigotimes_{j=i+1}^{m} \mathbf{A}_{n_{j}}^{(j)}\right)\right\|
$$


Proof. The usual insertion-triangle inequality argument for estimating differences of products yields, upon using $\left[76\right.$ and the definition of the constants $C_{\tilde{\mathbf{A}}}^{(i)}$,

$$
\begin{aligned}
\|\mathbf{A} \mathbf{v}-\tilde{\mathbf{A}} \mathbf{v}\| \leq \| & \sum_{n_{1}}\left(\mathbf{A}_{n_{1}}^{(1)}-\tilde{\mathbf{A}}_{n_{1}}^{(1)}\right) \otimes\left(\sum_{\check{\mathrm{n}}_{1}} c_{n} \mathbf{A}_{n_{2}}^{(2)} \otimes \cdots \otimes \mathbf{A}_{n_{m}}^{(m)}\right) \mathbf{v} \| \\
& +\ldots+\left\|\sum_{n_{m}}\left(\sum_{\check{\mathbf{n}}_{m}} c_{n} \tilde{\mathbf{A}}_{n_{1}}^{(1)} \otimes \cdots \otimes \tilde{\mathbf{A}}_{n_{m}-1}^{(m-1)}\right) \otimes\left(\mathbf{A}_{n_{m}}^{(m)}-\tilde{\mathbf{A}}_{n_{m}}^{(m)}\right) \mathbf{v}\right\| \\
\leq & C_{\tilde{\mathbf{A}}}^{(1)} \sum_{n_{1}}\left\|\left[\left(\mathbf{A}_{n_{1}}^{(1)}-\tilde{\mathbf{A}}_{n_{1}}^{(1)}\right) \otimes \mathrm{I} \otimes \cdots \otimes \mathrm{I}\right] \mathbf{v}\right\| \\
& +\ldots+C_{\tilde{\mathbf{A}}}^{(m)} \sum_{n_{m}}\left\|\left[\mathrm{I} \otimes \cdots \otimes \mathrm{I} \otimes\left(\mathbf{A}_{n_{m}}^{(m)}-\tilde{\mathbf{A}}_{n_{m}}^{(m)}\right)\right] \mathbf{v}\right\| .
\end{aligned}
$$

The assertion (81) follows now, using (79), from

$$
\begin{aligned}
\left\|\left[\left(\mathbf{A}_{n_{1}}^{(1)}-\tilde{\mathbf{A}}_{n_{1}}^{(1)}\right) \otimes \mathrm{I} \otimes \cdots \otimes \mathrm{I}\right] \mathbf{v}\right\| & \leq \sum_{p}\left\|\left[\left(\mathbf{A}_{n_{1}}^{(1)}-\tilde{\mathbf{A}}_{n_{1},[p]}^{(1)}\right) \mathrm{R}_{\Lambda_{\left.n_{1}, p\right]}^{(1)}} \otimes \mathrm{I} \otimes \cdots \otimes \mathrm{I}\right] \mathbf{v}\right\| \\
& \leq \sum_{p} \varepsilon_{n_{1}, p}^{(1)}\left\|\mathrm{R}_{\Lambda_{n_{1},[p]}^{(1)}} \pi^{(1)}(\mathbf{v})\right\|
\end{aligned}
$$

and analogous estimates for the other summands.

Remark 6. The constants $C_{\tilde{\mathbf{A}}}^{(i)}$, depending on the operator and its approximation, may introduce a dependence on $m$. In certain cases, this dependence is exponential. For instance, in the case of an operator of the form $\mathbf{A}=\mathbf{B} \otimes \mathbf{B} \otimes \cdots \otimes \mathbf{B}$ with $\|\tilde{\mathbf{B}}\| \leq\|\mathbf{B}\|$, we obtain $C_{\tilde{\mathbf{A}}}^{(i)}=\|\mathbf{B}\|^{m-1}$. This constant can therefore also strongly depend on an appropriate scaling of the problem under consideration. However, in the case of an operator

$$
\mathbf{A}=\mathbf{B} \otimes \mathrm{I} \otimes \cdots \otimes I+I \otimes \mathbf{B} \otimes \mathrm{I} \otimes \cdots \otimes I+\ldots+\mathrm{I} \otimes \cdots \otimes I \otimes \mathbf{B},
$$

we obtain instead $C_{\tilde{\mathbf{A}}}^{(i)} \leq(m-1)\|\mathbf{B}\|$. The dependence on $m$ of $C_{\tilde{\mathbf{A}}}^{(i)}$ is thus related to the number of factors in each summand of $\mathbf{A}$ that are not identity operators.

The partitions $\Lambda_{n_{i}}^{(i)}$ will later be identified for a class of matrices studied in the context of wavelet methods 8,32 .

Definition 7. Let $\Lambda$ be a countable index set and let $s^{*}>0$. We call an operator B: $\ell_{2}(\Lambda) \rightarrow \ell_{2}(\Lambda) s^{*}$-compressible if for any $0<s<s^{*}$, there exist summable positive sequences $\left(\alpha_{j}\right)_{j \geq 0},\left(\beta_{j}\right)_{j \geq 0}$ and for each $j \geq 0$, there exists $\mathbf{B}_{j}$ with at most $\alpha_{j} 2^{j}$ nonzero entries per row and column, such that $\left\|\mathbf{B}-\mathbf{B}_{j}\right\| \leq \beta_{j} 2^{-s j}$. For a given $s^{*}$-compressible operator $\mathbf{B}$, we denote the corresponding sequences by $\alpha(\mathbf{B}), \beta(\mathbf{B})$.

Moreover, we say that a family of operators $\{\mathbf{B}(n)\}_{n}$ is equi-s* ${ }^{*}$-compressible if all $\mathbf{B}(n)$ are $s^{*}$-compressible with the same choice of sequences $\left(\alpha_{j}\right),\left(\beta_{j}\right)$ and in addition, for all $\lambda \in \Lambda$ the number of nonzero elements in the rows and columns of the approximations $\mathbf{B}(n)_{j}$ can be estimated jointly for all $n$ in the form

$$
\#\left(\bigcup_{n}\left\{\lambda^{\prime} \in \Lambda:\left(\mathbf{B}(n)_{j}\right)_{\lambda, \lambda^{\prime}} \neq 0 \vee\left(\mathbf{B}(n)_{j}\right)_{\lambda^{\prime}, \lambda} \neq 0\right\}\right) \leq \alpha_{j} 2^{j} .
$$

Example 4. To give a structural example, let us assume that $\left\{\psi_{\lambda}\right\}_{\lambda \in \nabla}$ is an orthonormal wavelet basis on $\mathbb{R}$. As before, let $|\lambda|$ denote the level of the basis function $\psi_{\lambda}$. For $c, \sigma, \beta>0$, we denote by $\mathcal{M}_{c, \sigma, \beta}$ the class of infinite matrices for which

$$
\left|b_{\lambda, \lambda^{\prime}}\right| \leq c 2^{-|| \lambda|-| \lambda^{\prime}|| \sigma}\left(1+\operatorname{dist}\left(\operatorname{supp} \psi_{\lambda}, \operatorname{supp} \psi_{\lambda^{\prime}}\right)\right)^{-\beta} .
$$


Such bounds are known to hold, for instance, for wavelet representations of the double layer potential operator. Again, with a suitable rescaling of the wavelets, the representations of other potential types, as well as elliptic partial differential operators, exhibit the same decay of entries. It is shown in [8, Proposition 3.4] that (when specialized to the present case of one-dimensional factors) any $\mathbf{B} \in \mathcal{M}_{c, \sigma, \beta}$ with $\sigma>1 / 2, \beta>1$ is $s^{*}$-compressible with $s^{*}=\min \{\sigma-1 / 2, \beta-1\}$.

If $\mathbf{B}(n) \in \mathcal{M}_{c(n), \sigma(n), \beta(n)}$ with $c(n)$ and $\sigma(n)^{-1}, \beta(n)^{-1}$ uniformly bounded, then from the construction in the proof of [8, Proposition 3.4] it can be seen that the $\mathbf{B}(n)$ are equi- $s^{*}$-compressible with $s^{*}=\min \left\{\inf _{n} \sigma(n)-1 / 2, \inf _{n} \beta(n)-1\right\}$, since the same set of nonzero matrix entries can be used for each $n$.

The key property of $s^{*}$-compressible matrices in the context of adaptive methods is that they are not only bounded in $\ell_{2}$ but also on the smaller approximation spaces, and thus preserve sparsity in a quantifiable manner. We wish to establish such concepts next for the tensor setting.

To this end, assume that the components $\mathbf{A}_{n_{i}}^{(i)}$ in $\mathbf{A}$, given by (78), are $s^{*}$-compressible, and let $\mathbf{A}_{n_{i}, j}^{(i)}$ be the corresponding approximations according to Definition 7. Quite in the spirit of the adaptive application of an operator in wavelet coordinates (see [8]), for approximating $\mathbf{A} \mathbf{v}$ for given $\mathbf{v} \in \ell_{2}\left(\nabla^{d}\right)$, the a-priori knowledge about $\mathbf{A}$ in terms of $s^{*}$-compressibility is to be combined with a-posteriori information on $\mathbf{v}$. In fact, given $\mathbf{v} \in \ell_{2}\left(\nabla^{d}\right)$, we describe now how to construct for any $J \in \mathbb{N}$ approximations $\mathbf{w}_{J}$ to the sequence Av as follows. For each $i$ and for $j \in \mathbb{N}$, let $\bar{\Lambda}_{j}^{(i)}$ be the support of the best $2^{j}$ term approximation of $\pi^{(i)}(\mathbf{v})$ so that, in particular, $\bar{\Lambda}_{p}^{(i)} \subset \bar{\Lambda}_{p+1}^{(i)}$. If $\mathbf{A}_{n_{i}}^{(i)}=\mathrm{I}$, we simply set $\tilde{\mathbf{A}}_{n_{i}}^{(i)}=$ I. If $\mathbf{A}_{n_{i}}^{(i)} \neq \mathrm{I}$, we let $\bar{\Lambda}_{-1}^{(i)}:=\emptyset$ and

$$
\Lambda_{[p]}^{(i)}:= \begin{cases}\bar{\Lambda}_{p}^{(i)} \backslash \bar{\Lambda}_{p-1}^{(i)}, & p=0, \ldots, J \\ \nabla^{d_{i}} \backslash \bar{\Lambda}_{J}^{(i)}, & p=J+1 \\ \emptyset, & p>J+1 .\end{cases}
$$

Moreover, let

$$
\tilde{\mathbf{A}}_{n_{i},[p]}^{(i)}:= \begin{cases}\mathbf{A}_{n_{i}, J-p}^{(i)}, & p=0, \ldots, J \\ 0, & p>J .\end{cases}
$$

Note that, as a simple consequence of Definition 7, the $\tilde{\mathbf{A}}_{n_{i}}^{(i)}$ formed according to 80 are bounded independently of $\mathbf{v}$.

Lemma 5. Assume that the components $\mathbf{A}_{n_{i}}^{(i)}$ of $\mathbf{A}$ as in $(78)$ are $s^{*}$-compressible. Given any $\mathbf{v} \in \ell_{2}\left(\nabla^{d}\right), J \in \mathbb{N}$, let

$$
\tilde{\mathbf{A}}_{J}:=\sum_{\mathrm{n} \in \mathrm{K}_{m}(\mathrm{R})} c_{\mathrm{n}} \bigotimes_{i=1}^{m} \tilde{\mathbf{A}}_{n_{i}}^{(i)}, \quad \tilde{\mathbf{A}}_{n_{i}}^{(i)}:=\sum_{p \in \mathbb{N}_{0}} \tilde{\mathbf{A}}_{n_{i},[p]}^{(i)} \mathrm{R}_{\Lambda_{n_{i},[p]}^{(i)}}
$$

with $\mathrm{R}_{\Lambda_{n_{i},[p]}^{(i)}}, \tilde{\mathbf{A}}_{n_{i}}^{(i)}$ defined by 82 , 83), respectively. Then, whenever $\pi^{(i)}(\mathbf{v}) \in \mathcal{A}^{s}$ for some $0<s<s^{*}$, the finitely supported sequence $\mathbf{w}_{J}:=\tilde{\mathbf{A}}_{J} \mathbf{v}$ satisfies

$$
\left\|\mathbf{A} \mathbf{v}-\tilde{\mathbf{A}}_{J} \mathbf{v}\right\| \leq 2^{-s J} \sum_{i=1}^{m} C_{\tilde{\mathbf{A}}}^{(i)} R_{i}\left(\max _{n}\left\|\mathbf{A}_{n}^{(i)}\right\|+\left\|\hat{\beta}^{(i)}\right\|_{\ell_{1}}\right)\left\|\pi^{(i)}(\mathbf{v})\right\|_{\mathcal{A}^{s}},
$$

as well as

$$
\# \operatorname{supp}_{i} \tilde{\mathbf{A}}_{J} \mathbf{v} \leq R_{i}\left\|\hat{\alpha}^{(i)}\right\|_{\ell_{1}} 2^{J}
$$


where the sequences $\hat{\alpha}, \hat{\beta}$ are defined as the componentwise maxima of the sequences in Definition 7 for each $\mathbf{A}_{n_{i}}^{(i)}$, that is,

$$
\hat{\alpha}_{j}^{(i)}:=\max _{n} \alpha_{j}\left(\mathbf{A}_{n}^{(i)}\right), \quad \hat{\beta}_{j}^{(i)}:=\max _{n} \beta_{j}\left(\mathbf{A}_{n}^{(i)}\right) .
$$

Proof. We apply Lemma 4 with $\tilde{\mathbf{A}}_{n_{i},[p]}^{(i)}$ as in 83 and $\Lambda_{n_{i},[p]}^{(i)}:=\Lambda_{[p]}^{(i)}$, according to 82 . By $s^{*}$-compressibility, we have $\left\|\mathbf{A}_{n_{i}}^{(i)}-\tilde{\mathbf{A}}_{n_{i},[p]}^{(i)}\right\| \leq \hat{\beta}_{J-p}^{(i)} 2^{-s(J-p)}=: \varepsilon_{n_{i}, p}^{(i)}, p=0, \ldots, J$, $\left\|\mathbf{A}_{n_{i}}^{(i)}-\tilde{\mathbf{A}}_{n_{i},[J+1]}^{(i)}\right\|=\left\|\mathbf{A}_{n_{i}}^{(i)}\right\|,\left\|\mathrm{R}_{\Lambda_{[p]}^{(i)}} \pi^{(i)}(\mathbf{v})\right\|=0$ for $p>J+1$, and therefore

$$
\begin{aligned}
\left\|\mathbf{A} \mathbf{v}-\mathbf{w}_{J}\right\| \leq & \sum_{i=1}^{m} \sum_{n_{i}=1}^{R_{i}} C_{\tilde{\mathbf{A}}}^{(i)}\left\{\sum_{p=0}^{J} \hat{\beta}_{J-p}^{(i)} 2^{-s(J-p)}\left\|\mathrm{R}_{\Lambda_{n_{i},[p]}^{(i)}} \pi^{(i)}(\mathbf{v})\right\|\right. \\
& \left.+\left\|\mathbf{A}_{n_{i}}^{(i)}\right\|\left\|\mathrm{R}_{\Lambda_{n_{i},[J+1]}^{(i)}} \pi^{(i)}(\mathbf{v})\right\|\right\} .
\end{aligned}
$$

By the choice of the $\Lambda_{[p]}^{(i)}$ and the definition of $\|\cdot\|_{\mathcal{A}^{s}}$, we obtain $\left\|\mathrm{R}_{\Lambda_{[p]}^{(i)}} \pi^{(i)}(\mathbf{v})\right\| \leq 2^{-s p}\left\|\pi^{(i)}(\mathbf{v})\right\|_{\mathcal{A}^{s}}$ for $p=0, \ldots, J+1$, which confirms 84 . Furthermore,

$$
\# \operatorname{supp}_{i} \tilde{\mathbf{A}}_{J} \mathbf{v} \leq R_{i}\left(\hat{\alpha}_{J}^{(i)} 2^{J} 2^{0}+\hat{\alpha}_{J-1}^{(i)} 2^{J-1} 2^{1}+\ldots+\hat{\alpha}_{0}^{(i)} 2^{0} 2^{J}\right) \leq R_{i}\left\|\hat{\alpha}^{(i)}\right\|_{\ell_{1}} 2^{J},
$$

which is 85 .

Remark 7. Whenever $\mathbf{v}$ is finitely supported there exists a $p(\mathbf{v}) \in \mathbb{N}_{0}$ such that $\Lambda_{[p]}^{(i)}=\emptyset$ for $i=1, \ldots, m, p>p(\mathbf{v})$. Hence, the right hand side of (87) can be computed for each $J \in \mathbb{N}_{0}$, where the sum over $p$ terminates for $J \geq p(\mathbf{v})$ at $p(\mathbf{v})$. Further increasing $J$ will then decrease all summands on the right hand side of (87). Therefore, fixing any $s<s^{*}$ (close to $s^{*}$ ), we can find for any $\eta>0$ the integer $J(\eta)$ defined by

$$
\begin{aligned}
J(\eta):=\underset{J \in \mathbb{N}_{0}}{\arg \min }\{ & \sum_{i=1}^{m} \sum_{n_{i}=1}^{R_{i}} C_{\tilde{\mathbf{A}}}^{(i)}\left\{\sum_{p=0}^{J} \hat{\beta}_{J-p}^{(i)} 2^{-s(J-p)}\left\|\mathrm{R}_{\Lambda_{n_{i},[p]}^{(i)}} \pi^{(i)}(\mathbf{v})\right\|\right. \\
& \left.\left.+\left\|\mathbf{A}_{n_{i}}^{(i)}\right\|\left\|\mathrm{R}_{\Lambda_{n_{i},[J+1]}^{(i)}} \pi^{(i)}(\mathbf{v})\right\|\right\} \leq \eta\right\} .
\end{aligned}
$$

To further examine the properties of $\tilde{\mathbf{A}}_{J(\eta)} \mathbf{v}$ for a given finitely supported $\mathbf{v}$ let

$$
C_{\hat{\alpha}}^{(i)}:=\left\|\hat{\alpha}^{(i)}\right\|_{\ell_{1}}, \quad C_{\hat{\beta}}^{(i)}:=\left(\max _{n}\left\|\mathbf{A}_{n}^{(i)}\right\|+\left\|\hat{\beta}^{(i)}\right\|_{\ell_{1}}\right) .
$$

Theorem 8. Under the assumptions of Lemma 5 on $\mathbf{A}$ and any given finitely supported $\mathbf{v} \in \ell_{2}\left(\nabla^{d}\right)$, for any $\eta>0$ let

$$
\mathbf{w}_{\eta}:=\tilde{\mathbf{A}}_{J(\eta)} \mathbf{v}=: \tilde{\mathbf{A}}_{\eta} \mathbf{v},
$$

where $J(\eta)$ is defined by 89 . Then

$$
\begin{aligned}
&\left\|\mathbf{A} \mathbf{v}-\tilde{\mathbf{A}}_{\eta} \mathbf{v}\right\| \leq \eta \\
& \# \operatorname{supp}_{i}\left(\tilde{\mathbf{A}}_{\eta} \mathbf{v}\right) \leq 2 C_{\hat{\alpha}}^{(i)} R_{i} \eta^{-\frac{1}{s}}\left(\sum_{j=1}^{m} C_{\hat{\beta}}^{(j)} C_{\tilde{\mathbf{A}}}^{(j)} R_{j}\left\|\pi^{(j)}(\mathbf{v})\right\|_{\mathcal{A}^{s}}\right)^{\frac{1}{s}}, \\
&\left\|\pi^{(i)}\left(\tilde{\mathbf{A}}_{\eta} \mathbf{v}\right)\right\|_{\mathcal{A}^{s}} \leq \frac{2^{2 s+1}}{2^{s}-1}\left(C_{\hat{\alpha}}^{(i)}\right)^{s} C_{\hat{\beta}}^{(i)} C_{\tilde{\mathbf{A}}}^{(i)} R_{i}^{1+s}\left\|\pi^{(i)}(\mathbf{v})\right\|_{\mathcal{A}^{s}}
\end{aligned}
$$




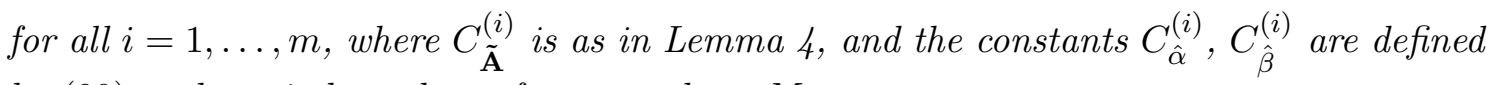
by 90 and are independent of $\mathbf{v}, \eta$, and $m$. Moreover,

$$
\operatorname{rank}_{i}(\tilde{\mathbf{A}} \mathbf{v}) \leq R_{i} \operatorname{rank}_{i}(\mathbf{v}), \quad i=1, \ldots, m .
$$

Proof. (92) follows from (89). The bound (93) is an immediate consequence of (85). Choosing for a given finitely supported $\mathbf{v}$ the mode frame system $\mathbb{U}=\mathbb{U}(\mathbf{v})$ according to HOSVD, 95) is clear, since with $\mathbf{U}^{(i)}$ and $\mathbf{a}$ as in Lemma 4 , we obtain

$$
\tilde{\mathbf{A}} \mathbf{v}=\sum_{\mathrm{n} \in \mathrm{K}_{m}(\mathrm{R})} \sum_{\mathrm{k} \in \mathbb{N}^{m}} \mathbf{d}_{\left(n_{1}, k_{1}\right), \ldots,\left(n_{m}, k_{m}\right)} \bigotimes_{i=1}^{m} \tilde{\mathbf{A}}_{n_{i}}^{(i)} \mathbf{U}_{k_{i}}^{(i)},
$$

where $\mathbf{d}_{\left(n_{1}, k_{1}\right), \ldots,\left(n_{m}, k_{m}\right)}=c_{\mathrm{n}} a_{\mathrm{k}}$.

Without loss of generality it suffices to prove (94) only for $i=1$, which allows us to temporarily simplify the notation by writing $\Lambda_{[p]}$ for $\Lambda_{[p]}^{(1)}$. Note first that for each $\nu_{1} \in \nabla^{d_{1}}$, using Proposition 3 followed by (77), we obtain

$$
\begin{aligned}
\pi_{\nu_{1}}^{(1)}(\tilde{\mathbf{A}} \mathbf{v}) & \leq C_{\tilde{\mathbf{A}}}^{(1)} \sum_{n_{1}=1}^{R_{1}} \pi_{\nu_{1}}^{(1)}\left(\tilde{\mathbf{A}}_{n_{1}}^{(1)} \otimes \mathbf{I} \cdots \otimes \mathbf{I} \mathbf{v}\right) \\
& =C_{\tilde{\mathbf{A}}}^{(1)} \sum_{n_{1}=1}^{R_{1}}\left(\sum_{k}\left|\sigma_{k}^{(1)}\right|^{2}\left|\left(\tilde{\mathbf{A}}_{n_{1}}^{(1)} \mathbf{U}_{k}^{(1)}\right)_{\nu_{1}}\right|^{2}\right)^{\frac{1}{2}}
\end{aligned}
$$

where we have used $(53)$ in the last step for the mode frame system $\mathbb{U}(\mathbf{v})$ from Theorem 2. In order to bound next the terms on the right hand side of 97) let

$$
\begin{aligned}
& \hat{\Lambda}_{n_{1},[0]}:=\text { supp range } \mathbf{A}_{n_{1}, 0}^{(1)} \mathrm{R}_{\Lambda_{[0]}}, \\
& \hat{\Lambda}_{n_{1},[q]}:=\left(\bigcup_{j+\ell=q} \text { supp range } \mathbf{A}_{n_{1}, j}^{(1)} \mathrm{R}_{\Lambda_{[\ell]}}\right) \backslash\left(\bigcup_{i<q} \hat{\Lambda}_{n_{1},[i]}\right), \quad q>0 .
\end{aligned}
$$

By the same argument as in 88 , we also obtain

$$
\# \hat{\Lambda}_{n_{1},[q]} \leq\left\|\hat{\alpha}^{(1)}\right\|_{\ell_{1}} 2^{q} .
$$

Since for $q=1, \ldots, J$, and each $k$, we have

$$
\left\|\mathrm{R}_{\hat{\Lambda}_{n_{1},[q]}} \tilde{\mathbf{A}}_{n_{1}}^{(1)} \mathbf{U}_{k}^{(1)}\right\| \leq \sum_{\ell=0}^{q}\left\|\mathrm{R}_{\hat{\Lambda}_{n_{1},[q]}} \tilde{\mathbf{A}}_{n_{1}}^{(1)} \mathrm{R}_{\Lambda_{[\ell]}} \mathbf{U}_{k}^{(1)}\right\|,
$$

and since by $(83), \mathrm{R}_{\hat{\Lambda}_{n_{1},[q]}} \tilde{\mathbf{A}}_{n_{1}}^{(1)} \mathrm{R}_{\Lambda_{[\ell]}}=\mathrm{R}_{\hat{\Lambda}_{n_{1},[q]}}\left(\mathbf{A}_{n_{1}, J-\ell}^{(1)}-\mathbf{A}_{n_{1}, q-\ell+1}^{(1)}\right) \mathrm{R}_{\Lambda_{[\ell]}}$, this can be estimated further by

$$
\begin{aligned}
\left\|\mathrm{R}_{\hat{\Lambda}_{n_{1},[q]}} \tilde{\mathbf{A}}_{n_{1}}^{(1)} \mathbf{U}_{k}^{(1)}\right\| & \leq \sum_{\ell=0}^{q}\left(\left\|\mathbf{A}_{n_{1}}^{(1)}-\mathbf{A}_{n_{1}, J-\ell}^{(1)}\right\|+\left\|\mathbf{A}_{n_{1}}^{(1)}-\mathbf{A}_{n_{1}, q-\ell-1}^{(1)}\right\|\right)\left\|\mathrm{R}_{\Lambda_{[\ell]}} \mathbf{U}_{k}^{(1)}\right\| \\
& \leq \sum_{\ell=0}^{q}\left(\hat{\beta}_{J-\ell}^{(1)} 2^{-s(J-\ell)}+\hat{\beta}_{q-\ell-1}^{(1)} 2^{-s(q-\ell-1)}\right)\left\|\mathrm{R}_{\Lambda_{[\ell]}} \mathbf{U}_{k}^{(1)}\right\| \\
& \leq\left(2\left\|\hat{\beta}^{(1)}\right\|_{\ell_{1}}\right)^{\frac{1}{2}}\left(\sum_{\ell=0}^{q}\left(\hat{\beta}_{J-\ell}^{(1)}+\hat{\beta}_{q-\ell-1}^{(1)}\right) 2^{-2 s(q-\ell-1)}\left\|\mathrm{R}_{\Lambda_{[\ell]}} \mathbf{U}_{k}^{(1)}\right\|^{2}\right)^{\frac{1}{2}}
\end{aligned}
$$


Hence, abbreviating $\gamma_{\ell}:=\hat{\beta}_{J-\ell}^{(1)}+\hat{\beta}_{q-\ell-1}^{(1)}$, we obtain

$$
\begin{aligned}
& \left\|\mathrm{R}_{\hat{\Lambda}_{n_{1},[q]}} \pi^{(1)}\left(\tilde{\mathbf{A}}_{n_{1}}^{(1)} \otimes \mathrm{I}_{2} \cdots \otimes \mathrm{I}_{m} \mathbf{v}\right)\right\|^{2} \\
& \quad \leq 2^{2 s+1}\left\|\hat{\beta}^{(1)}\right\|_{\ell_{1}} \sum_{k}\left|\sigma_{k}^{(1)}\right|^{2} \sum_{\ell=0}^{q} \gamma_{\ell} 2^{-2 s(q-\ell)}\left\|\mathrm{R}_{\Lambda_{[\ell]}} \mathbf{U}_{k}^{(1)}\right\|^{2} \\
& \quad=2^{2 s+1}\left\|\hat{\beta}^{(1)}\right\|_{\ell_{1}} \sum_{\ell=0}^{q} \gamma_{\ell} 2^{-2 s(q-\ell)}\left\|\mathrm{R}_{\Lambda_{[\ell]}} \pi^{(1)}(\mathbf{v})\right\|^{2} \\
& \quad \leq 2^{2 s+1}\left\|\hat{\beta}^{(1)}\right\|_{\ell_{1}} 2^{-2 s q}\left\|\pi^{(1)}(\mathbf{v})\right\|_{\mathcal{A}^{s}}^{2} \sum_{\ell=0}^{q} \gamma_{\ell},
\end{aligned}
$$

and thus

$$
\left\|\mathrm{R}_{\hat{\Lambda}_{n_{1},[q]}} \pi^{(1)}\left(\tilde{\mathbf{A}}_{n_{1}}^{(1)} \otimes \mathrm{I} \cdots \otimes \mathbf{I v}\right)\right\| \leq 2^{-s q} 2^{s+1}\left\|\hat{\beta}^{(1)}\right\|_{\ell_{1}}\left\|\pi^{(1)}(\mathbf{v})\right\|_{\mathcal{A}^{s}}
$$

Recall that the sets $\hat{\Lambda}_{n_{1},[q]}$ are disjoint with $\# \hat{\Lambda}_{n_{1},[q]} \leq\left\|\hat{\alpha}^{(1)}\right\|_{\ell_{1}} 2^{q}$. By definition of the $\mathcal{A}^{s}$-quasi-norm, we have

$$
\left\|\pi^{(1)}\left(\tilde{\mathbf{A}}_{n_{1}}^{(1)} \otimes \mathrm{I} \cdots \otimes \mathbf{I} \mathbf{v}\right)\right\|_{\mathcal{A}^{s}} \leq \sup _{q \in \mathbb{N}}\left(\sum_{j<q} \# \hat{\Lambda}_{n_{1},[j]}\right)^{s} \sum_{j \geq q}\left\|\mathrm{R}_{\hat{\Lambda}_{\left.n_{1}, j\right]}} \pi^{(1)}\left(\tilde{\mathbf{A}}_{n_{1}}^{(1)} \otimes \mathrm{I} \cdots \otimes \mathrm{I} \mathbf{v}\right)\right\| .
$$

Hence from 99 we infer

$$
\left\|\pi^{(1)}\left(\tilde{\mathbf{A}}_{n_{1}}^{(1)} \otimes \mathrm{I} \cdots \otimes \mathbf{I v}\right)\right\|_{\mathcal{A}^{s}} \leq\left(2^{2 s+1}\left(2^{s}-1\right)^{-1}\left\|\hat{\alpha}^{(1)}\right\|_{\ell_{1}}^{s}\left\|\hat{\beta}^{(1)}\right\|_{\ell_{1}}\right)\left\|\pi^{(1)}(\mathbf{v})\right\|_{\mathcal{A}^{s}} .
$$

Since by the first inequality in (97), we have

$$
\left\|\pi^{(1)}(\tilde{\mathbf{A}} \mathbf{v})\right\|_{\mathcal{A}^{s}} \leq C_{\tilde{\mathbf{A}}}^{(1)} R_{1}^{s} \sum_{n_{1}=1}^{R_{1}}\left\|\pi^{(1)}\left(\tilde{\mathbf{A}}_{n_{1}}^{(1)} \otimes \mathrm{I} \cdots \otimes \mathbf{I} \mathbf{v}\right)\right\|_{\mathcal{A}^{s}}
$$

we arrive at (94).

Remark 8. The estimate 94 corresponds to the worst case that the sets $\hat{\Lambda}_{n_{i},[q]}$ constructed in the proof are disjoint for different $n_{i}$. If, on the contrary, the $\left\{\mathbf{A}_{n_{i}}^{(i)}\right\}_{n_{i}}$ are equi-s*-compressible, and hence these sets are the same for all $n_{i}$, we can combine (97) directly with (99) to obtain instead

$$
\left\|\pi^{(i)}(\tilde{\mathbf{A}} \mathbf{v})\right\|_{\mathcal{A}^{s}} \lesssim\left(C_{\hat{\alpha}}^{(i)}\right)^{s} C_{\hat{\beta}}^{(i)} C_{\tilde{\mathbf{A}}}^{(i)} R_{i}\left\|\pi^{(i)}(\mathbf{v})\right\|_{\mathcal{A}^{s}}
$$

i.e., an improvement by a factor $R_{i}^{s}$. Similarly, in this case we also obtain that by a modification of (88), the estimate (93) can be replaced by

$$
\# \operatorname{supp}_{i}(\tilde{\mathbf{A}} \mathbf{v}) \lesssim C_{\hat{\alpha}}^{(i)} \eta^{-\frac{1}{s}}\left(\sum_{j=1}^{m} C_{\hat{\beta}}^{(j)} C_{\tilde{\mathbf{A}}}^{(j)} R_{j}\left\|\pi^{(j)}(\mathbf{v})\right\|_{\mathcal{A}^{s}}\right)^{\frac{1}{s}} .
$$

Remark 9. If $r_{i}:=\operatorname{rank}_{i}(\mathbf{v})<\infty$, the number ops $(\tilde{\mathbf{A}} \mathbf{v})$ of arithmetic operations for evaluating $\tilde{\mathbf{A}} \mathbf{v}$ as in Lemma 8, for a given HOSVD of $\mathbf{v}$, can be estimated by

$$
\operatorname{ops}(\tilde{\mathbf{A}} \mathbf{v}) \lesssim \prod_{i=1}^{m} R_{i} r_{i}+\eta^{-\frac{1}{s}} \sum_{i=1}^{m} C_{\hat{\alpha}}^{(i)} R_{i} r_{i}\left(\sum_{j=1}^{m} C_{\hat{\beta}}^{(j)} C_{\tilde{\mathbf{A}}}^{(j)} R_{j}\left\|\pi^{(j)}(\mathbf{v})\right\|_{\mathcal{A}^{s}}\right)^{\frac{1}{s}}
$$

with a constant independent of $\mathbf{v}, \eta$, and $m$. 
Proof. The sorting of entries of $\pi^{(i)}(\mathbf{v})$ required for obtaining the index sets of best $2^{j}$ term approximations in Theorem 8 can be replaced by an approximate sorting by binary binning, requiring only $\# \operatorname{supp}_{i}(\mathbf{v})$ operations, as suggested in [4, 26]. This only leads to a change in the generic constants in the resulting estimates.

Let $\mathbf{v}$ have the HOSVD $\mathbf{v}=\sum_{k} a_{k} \mathbb{U}_{k}$, then, on the one hand, we need to form the core tensor for the result, which takes $\prod_{i=1}^{m} R_{i} r_{i}$ operations, and evaluate the approximations to $\tilde{\mathbf{A}}_{n_{i}}^{(i)} \mathbf{U}_{k_{i}}^{(i)}$ for $n_{i}=1, \ldots, R_{i}$ and $k_{i}=1, \ldots, r_{i}$. The number of operations for each of these terms can be estimated as in [8, which leads to (100).

As the first term on the right hand side of 100 shows, the Tucker format still suffers from the curse of dimensionality due to the complexity of the core tensors.

\subsubsection{Hierarchical Tucker Format}

For applying operators to coefficient sequences given in the hierarchical Tucker format, we need a representation of these operators with analogous hierarchical structure. That is, in the representation

$$
\mathbf{A}=\sum_{\mathrm{n} \in \mathrm{K}_{m}(\mathrm{R})} c_{\mathrm{n}} \bigotimes_{i=1}^{m} \mathbf{A}_{n_{i}}^{(i)},
$$

for the finitely supported tensor $\mathbf{c}=\left(c_{\mathrm{n}}\right) \in \ell_{2}\left(\mathbb{N}^{m}\right)$ we need in addition a hierarchical decomposition

$$
\mathbf{c}=\Sigma_{\mathcal{D}_{m}}\left(\left\{\mathbf{C}^{(\alpha, \nu)}: \alpha \in \mathcal{N}\left(\mathcal{D}_{m}\right), \nu \in \mathbb{N}\right\}\right),
$$

see (35), (36). Here for $\alpha \in \mathcal{N}\left(\mathcal{D}_{m}\right)$, we extend the definition of representation ranks in the representation of $\mathbf{A}$ to each $\alpha \in \mathcal{D}_{m}$ by setting $R_{\{i\}}:=R_{i}$ and

$$
R_{\alpha}:=\#\left\{\nu: \mathbf{C}^{(\alpha, \nu)} \neq 0\right\} .
$$

In what follows, we assume $\max _{\alpha \in \mathcal{D}_{m}} R_{\alpha}<\infty$ and $R_{0_{m}}=1$. According to Theorem 4 . $\mathbf{v} \in \ell_{2}\left(\nabla^{d}\right)$ has a representation

$$
\mathbf{v}=\sum_{\mathbf{k} \in \mathbb{N}^{m}} a_{\mathbf{k}} \mathbb{U}_{\mathbf{k}}, \quad \mathbf{a}=\Sigma_{\mathcal{D}_{m}}\left(\left\{\mathbf{B}^{(\alpha, k)}\right\}\right) .
$$

If $\max _{\alpha \in \mathcal{D}_{m}} \operatorname{rank}_{\alpha}(\mathbf{v})<\infty$, then $\tilde{\mathbf{A}} \mathbf{v}$ can be represented in the form (96), with $\mathbf{d}$ again admitting a hierarchical representation in terms of matrices $\mathbf{D}^{(\alpha,(\nu, k)))}$ on $\mathbb{N}^{2} \times \mathbb{N}^{2}$ with entries

$$
\mathbf{D}_{\left(\left(\mu_{1}, l_{1}\right),\left(\mu_{2}, l_{2}\right)\right)}^{(\alpha, \nu, k))}:=\mathbf{C}_{\left(\mu_{1}, \mu_{2}\right)}^{(\alpha, \nu)} \mathbf{B}_{l_{1}, l_{2}}^{(\alpha, k)} .
$$

That is, as in (35), we have an explicit representation

$$
\mathbf{d}=\Sigma_{\mathcal{D}_{m}}\left(\left\{\mathbf{D}^{(\alpha,(\nu, k)))}: \alpha \in \mathcal{N}\left(\mathcal{D}_{m}\right), \nu=1, \ldots, R_{\alpha}, k=1, \ldots, \operatorname{rank}_{\alpha}(\mathbf{v})\right\}\right)
$$

in (96), where the indices in $k \in \mathbb{N}$ are replaced in the definition of $\Sigma_{\mathcal{D}_{m}}(\cdot)$ by the indices $(\nu, k) \in \mathbb{N}^{2}$.

Example 5. To give a specific example, we consider an operator of the form

$$
\mathbf{A}_{1} \otimes \mathrm{I}_{2} \otimes \cdots \otimes \mathrm{I}_{m}+\ldots+\mathrm{I}_{1} \otimes \cdots \otimes \mathrm{I}_{m-1} \otimes \mathbf{A}_{m}
$$

in the hierarchical format with dimension tree

$$
\mathcal{D}_{m}=\left\{0_{m},\{1\},\{2, \ldots, m\},\{2\},\{3, \ldots, m\}, \ldots,\{m\}\right\}
$$


as in Example 2, Setting $\mathbf{A}_{1}^{(i)}=\mathrm{I}_{i}, \mathbf{A}_{2}^{(i)}=\mathbf{A}_{i}$ for $i=1, \ldots, m$, we obtain a representation as in 102 with $R_{\alpha}=2$ for $\alpha \neq 0_{m}$, and

$$
\mathbf{C}^{\left(0_{m}, 1\right)}=\left(\begin{array}{ll}
0 & 1 \\
1 & 0
\end{array}\right), \quad \mathbf{C}^{(\alpha, 1)}=\left(\begin{array}{ll}
1 & 0 \\
0 & 0
\end{array}\right), \mathbf{C}^{(\alpha, 2)}=\left(\begin{array}{ll}
0 & 1 \\
1 & 0
\end{array}\right), \alpha \in \mathcal{N}\left(\mathcal{D}_{m}\right) \backslash\left\{0_{m}\right\} .
$$

The estimates in Theorem 8 now directly carry over to the hierarchical Tucker format, where as the only modification, (95) is replaced by

$$
\operatorname{rank}_{\alpha}(\tilde{\mathbf{A}} \mathbf{v}) \leq R_{\alpha} \operatorname{rank}_{\alpha}(\mathbf{v}) .
$$

Remark 10. If for a given $\mathcal{H} S V D$ of $\mathbf{v}, r_{\alpha}:=\operatorname{rank}_{\alpha}(\mathbf{v})<\infty, \alpha \in \mathcal{N}\left(\mathcal{D}_{m}\right)$, the number $\operatorname{ops}(\tilde{\mathbf{A}} \mathbf{v})$ of arithmetic operations for evaluating $\tilde{\mathbf{A}} \mathbf{v}$ as in Theorem 8 can be estimated by

$$
\begin{aligned}
\operatorname{ops}(\tilde{\mathbf{A}} \mathbf{v}) \lesssim \sum_{\alpha \in \mathcal{N}\left(\mathcal{D}_{m}\right)} R_{\alpha} r_{\alpha} \prod_{q=1}^{2} & R_{\mathrm{c}_{q}(\alpha)} r_{\mathrm{c}_{q}(\alpha)} \\
& \quad+\eta^{-\frac{1}{s}} \sum_{i=1}^{m} C_{\hat{\alpha}}^{(i)} R_{i} r_{i}\left(\sum_{j=1}^{m} C_{\hat{\beta}}^{(j)} C_{\tilde{\mathbf{A}}}^{(j)} R_{j}\left\|\pi^{(j)}(\mathbf{v})\right\|_{\mathcal{A}^{s}}\right)^{\frac{1}{s}},
\end{aligned}
$$

with a constant independent of $\mathbf{v}, \eta$, and $m$.

Comparing the first summand on the right hand side of (104) to the one in (100), we observe a substantial reduction in complexity regarding the dependence on $m$ (and hence d).

\subsection{Low-Rank Approximations of Operators}

In many applications of interest, the involved operators do not have an explicit low-rank form, but there exist efficient approximations to these operators in low-rank representation.

Such a case can be handled by replacing a given operator $\mathbf{A}$ by such an approximation and then applying the construction for operators given in low-rank form as in the previous subsections.

To make this precise, we assume that for a suitable growth sequence $\gamma_{\mathbf{A}}$, there exist approximations $\mathbf{A}_{N}$ for $N \in \mathbb{N}$ with

$$
\sup _{N} \gamma_{\mathbf{A}}(N)\left\|\mathbf{A}-\mathbf{A}_{N}\right\|=: M_{\mathbf{A}}<\infty,
$$

where each $\mathbf{A}_{N}$ has a representation (101) with $R_{i} \leq N$. Moreover, in the case of the hierarchical Tucker format we assume in addition that $R_{\alpha} \leq N$ with $R_{\alpha}$ as in 103.

Moreover, we need to quantify the approximability of the $\mathbf{A}_{N}$. We assume that all tensor factors arising in each $\mathbf{A}_{N}$ are $s^{*}$-compressible, and that for the approximations $\tilde{\mathbf{A}}_{N}$ of $\mathbf{A}_{N}$ according to Lemma 4 and Theorem 8 - with constants $C_{\tilde{\mathbf{A}}_{N}}^{(i)}, C_{\hat{\alpha}_{N}}^{(i)}, C_{\hat{\beta}_{N}}^{(i)}$ in Theorem 8 - we have

$$
C_{\mathbf{A}, \tilde{\mathbf{A}}}:=\sup _{N}\left(\max _{i} C_{\hat{\alpha}_{N}}^{(i)}\right)^{s}\left(\max _{i} C_{\tilde{\mathbf{A}}_{N}}^{(i)} C_{\hat{\beta}_{N}}^{(i)}\right)<\infty .
$$

Under these conditions, we shall say that the approximations $\mathbf{A}_{N}$ to $\mathbf{A}$ are uniformly $s^{*}$-compressible.

Under this assumption, the estimates for ops $(\tilde{\mathbf{A}} \mathbf{v})$ obtained in Remarks 9 and 10 carry over to the present setting with additional low-rank approximation of the operator. Here 
for given $\eta>0$ and $\mathbf{v}$, we choose $N$ such that $\left\|\mathbf{A}-\mathbf{A}_{N}\right\| \leq \eta / 2$ and $\tilde{\mathbf{A}}_{N}$ such that $\left\|\mathbf{A}_{N} \mathbf{v}-\tilde{\mathbf{A}}_{N} \mathbf{v}\right\| \leq \eta / 2$, which in summary yields for the Tucker format

$$
\begin{aligned}
\operatorname{ops}(\tilde{\mathbf{A}} \mathbf{v}) \lesssim\left(\gamma_{\mathbf{A}}^{-1}\left(2 M_{\mathbf{A}} / \eta\right)\right)^{m} & \prod_{i=1}^{m} \operatorname{rank}_{i}(\mathbf{v}) \\
& +C_{\mathbf{A}, \tilde{\mathbf{A}}}^{\frac{1}{s}} \eta^{-\frac{1}{s}}\left(\gamma_{\mathbf{A}}^{-1}\left(2 M_{\mathbf{A}} / \eta\right)\right)^{1+s^{-1}} \sum_{i=1}^{m} \operatorname{rank}_{i}(\mathbf{v})\left(\sum_{j=1}^{m}\left\|\pi^{(j)}(\mathbf{v})\right\|_{\mathcal{A}^{s}}\right)^{\frac{1}{s}},
\end{aligned}
$$

and for the hierarchical Tucker format

$$
\begin{aligned}
& \operatorname{ops}(\tilde{\mathbf{A}} \mathbf{v}) \lesssim\left(\gamma_{\mathbf{A}}^{-1}\left(2 M_{\mathbf{A}} / \eta\right)\right)^{3} \sum_{\alpha \in \mathcal{N}\left(\mathcal{D}_{m}\right)} \operatorname{rank}_{\alpha}(\mathbf{v}) \prod_{q=1}^{2} \operatorname{rank}_{\mathrm{c}_{q}(\alpha)}(\mathbf{v}) \\
& +C_{\mathbf{A}, \tilde{\mathbf{A}}}^{\frac{1}{s}} \eta^{-\frac{1}{s}}\left(\gamma_{\mathbf{A}}^{-1}\left(2 M_{\mathbf{A}} / \eta\right)\right)^{1+s^{-1}} \sum_{i=1}^{m} \operatorname{rank}_{i}(\mathbf{v})\left(\sum_{j=1}^{m}\left\|\pi^{(j)}(\mathbf{v})\right\|_{\mathcal{A}^{s}}\right)^{\frac{1}{s}} .
\end{aligned}
$$

Note again the reduction in complexity in the first term of (108) over (107).

\section{An Adaptive Iterative Scheme}

\subsection{Formulation and Basic Convergence Properties}

We have now all prerequisites in place to formulate an adaptive method whose basic structure resembles the one introduced in $[9$ for linear operator equations $\mathbf{A u}=\mathbf{f}$, where $\mathbf{f} \in \ell_{2}$ and $\mathbf{A}$ is bounded and elliptic on $\ell_{2}$, that is,

$$
\langle\mathbf{A} \mathbf{v}, \mathbf{v}\rangle_{\ell_{2}} \geq \lambda_{\mathbf{A}}\|\mathbf{v}\|_{\ell_{2}}^{2}, \quad\|\mathbf{A v}\|_{\ell_{2}} \leq \Lambda_{\mathbf{A}}\|\mathbf{v}\|_{\ell_{2}}
$$

holds for fixed constants $\lambda_{\mathbf{A}}, \Lambda_{\mathbf{A}}>0$. The scheme can be regarded as a perturbation of a simple Richardson iteration,

$$
\mathbf{v}_{i+1}:=\mathbf{v}_{i}-\omega\left(\mathbf{A} \mathbf{v}_{i}-\mathbf{f}\right),
$$

which applies to both symmetric and nonsymmetric elliptic A. In both cases, the parameter $\omega>0$ can be chosen such that $\|\mathrm{I}-\omega \mathbf{A}\|<1$.

Based on the developments in the previous sections, we have at hand numerically realizable procedures APPLY, RHS, RECOMPRESS, and COARSEN, which for finitely supported $\mathbf{v}$ and any tolerance $\eta>0$ satisfy

$$
\begin{array}{ll}
\|\mathbf{A v}-\operatorname{APpty}(\mathbf{v} ; \eta)\| \leq \eta, & \|\mathbf{f}-\operatorname{RHS}(\eta)\| \leq \eta, \\
\|\mathbf{v}-\operatorname{RecompRess}(\mathbf{v} ; \eta)\| \leq \eta, & \|\mathbf{v}-\operatorname{COARSEn}(\mathbf{v} ; \eta)\| \leq \eta .
\end{array}
$$

Specifications of the complexities of these procedures will be summarized in $\$ 5.2$. The adaptive scheme that we analyze in what follows is given in Algorithm 5.1.

Proposition 5. Let the step size $\omega>0$ in Algorithm 5.1 satisfy $\|\mathbf{I}-\omega \mathbf{A}\| \leq \rho<1$. Then the intermediate steps $\mathbf{u}_{k}$ of Algorithm 5.1 satisfy $\left\|\mathbf{u}_{k}-\mathbf{u}\right\| \leq \theta^{k} \delta$, and in particular, the output $\mathbf{u}_{\varepsilon}$ of Algorithm 5.1 satisfies $\left\|\mathbf{u}_{\varepsilon}-\mathbf{u}\right\| \leq \varepsilon$. 


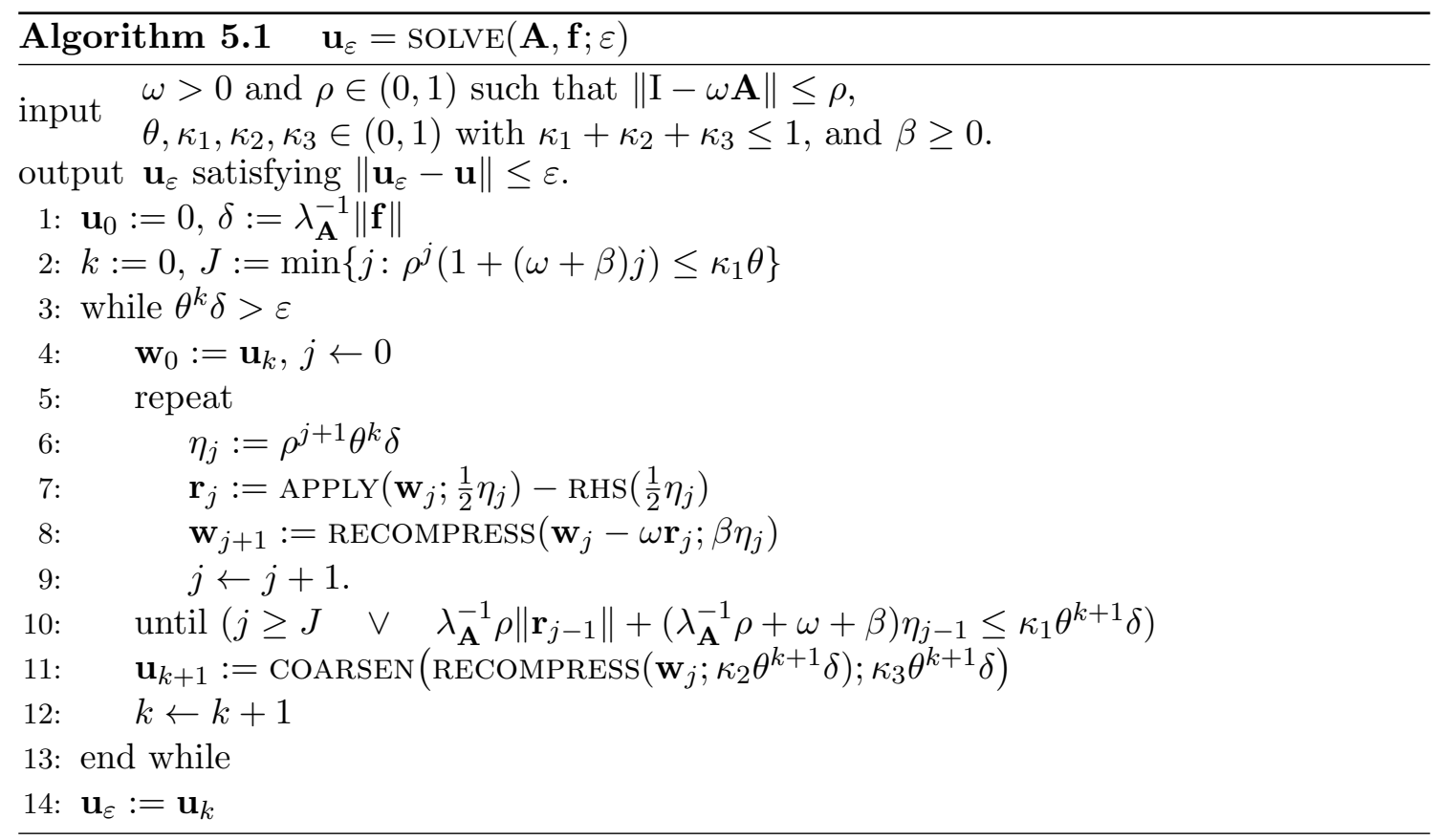

Proof. Since $\kappa_{1}+\kappa_{2}+\kappa_{3} \leq 1$, it suffices to show that for any $k$, after the termination of the inner loop the error bound

$$
\left\|\mathbf{w}_{j}-\mathbf{u}\right\| \leq \kappa_{1} \theta^{k+1} \delta
$$

holds. By the choice of $\omega$, we have

$$
\begin{aligned}
\left\|\mathbf{w}_{j+1}-\mathbf{u}\right\| & \leq\left\|(\mathrm{I}-\omega \mathbf{A})\left(\mathbf{w}_{j}-\mathbf{u}\right)\right\|+\omega\left\|\left(\mathbf{A} \mathbf{w}_{j}-f\right)-\mathbf{r}_{j}\right\| \\
& \leq \rho\left\|\mathbf{w}_{j}-\mathbf{u}\right\|+(\omega+\beta) \eta_{j},
\end{aligned}
$$

and recursive application of this estimate yields

$$
\left\|\mathbf{w}_{j}-\mathbf{u}\right\| \leq \rho^{j}\left\|\mathbf{w}_{0}-\mathbf{u}\right\|+(\omega+\beta) \sum_{l=0}^{j-1} \rho^{j-1-l} \eta_{l} \leq \rho^{j}(1+j(\omega+\beta)) \theta^{k} \delta .
$$

Thus on the one hand, if the inner loop exits with the first condition in line 10 , then (111) holds by definition of $J$. On the other hand, if the second condition is met, then (111) holds because

$$
\begin{aligned}
\left\|\mathbf{w}_{j}-\mathbf{u}\right\| & \leq \rho\left\|\mathbf{w}_{j-1}-\mathbf{u}\right\|+(\omega+\beta) \eta_{j-1} \\
& \leq \rho c_{\mathbf{A}}^{-1}\left(\left\|\mathbf{r}_{j-1}\right\|+\eta_{j-1}\right)+(\omega+\beta) \eta_{j-1} \leq \kappa_{1} \theta^{k+1} \delta .
\end{aligned}
$$

\subsection{Complexity}

Quite in the spirit of adaptive wavelet methods we analyze the performance of the above scheme by comparing it to an "optimality benchmark" addressing the following question: suppose the unknown solution exhibits a certain (unknown) rate of tensor approximability where the involved tensors have a certain (unknown) best $N$-term approximability with respect to their wavelet representations. Does the scheme automatically recover these rates? Thus, unlike the situation in wavelet analysis we are dealing here with two types of approximation, and the choice of corresponding rates as a benchmark model should, 
of course, be representative for relevant application scenarios. For the present complexity analysis, we focus on growth sequences of subexponential or exponential type for the involved low-rank approximations, combined with an algebraic approximation rate for the corresponding tensor mode frames. The rationale for this choice is the following. Approximation rates in classical methods are governed by the regularity of the approximand which, unless the approximand is analytic, results in algebraic rates suffering from the curse of dimensionality. However, functions of many variables may very well exhibit a high degree of tensor sparsity without being very regular in the Sobolev or Besov sense. Therefore, fast tensor-rates combined with polynomial rates for the compressibility of the mode frames mark an ideal target scenario for tensor methods, since, as will be shown, the curse of dimensionality can be significantly ameliorated without requiring excessive regularity.

The precise formulation of our benchmark model reads as follows.

Assumptions 1. Concerning the tensor approximability of $\mathbf{u}, \mathbf{A}$, and $\mathbf{f}$, we make the following assumptions:

(i) $\mathbf{u} \in \mathcal{A}_{\mathcal{H}}\left(\gamma_{\mathbf{u}}\right)$ with $\gamma_{\mathbf{u}}(n)=e^{d_{\mathbf{u}} n^{1 / b_{\mathbf{u}}}}$ for some $d_{\mathbf{u}}>0, b_{\mathbf{u}} \geq 1$.

(ii) A satisfies (105) for an $M_{\mathbf{A}}>0$, with $\gamma_{\mathbf{A}}(n)=e^{d_{\mathbf{A}} n^{1 / b} \mathbf{A}}$ where $d_{\mathbf{A}}>0, b_{\mathbf{A}} \geq 1$.

(iii) Furthermore, let $\mathbf{f} \in \mathcal{A}_{\mathcal{H}}\left(\gamma_{\mathbf{f}}\right)$ with $\gamma_{\mathbf{f}}(n)=e^{d_{\mathbf{f}} n^{1 / b_{\mathbf{f}}}}$, where $d_{\mathbf{f}}=\min \left\{d_{\mathbf{u}}, d_{\mathbf{A}}\right\}$ and $b_{\mathbf{f}}=b_{\mathbf{u}}+b_{\mathbf{A}}$.

Concerning the approximability of lower-dimensional components, we assume that for some $s^{*}>0$, we have the following:

(iv) $\pi^{(i)}(\mathbf{u}) \in \mathcal{A}^{s}$ for $i=1, \ldots, m$, for any $s$ with $0<s<s^{*}$.

(v) The low-rank approximations to $\mathbf{A}$ are uniformly $s^{*}$-compressible in the sense of Subsection 4.2, with $C_{\mathbf{A}}:=\sup _{\eta>0} C_{\mathbf{A}, \tilde{\mathbf{A}}}<\infty$, where $C_{\mathbf{A}, \tilde{\mathbf{A}}}$ is defined as in 106 for each value of $\eta$.

(vi) $\pi^{(i)}(\mathbf{f}) \in \mathcal{A}^{s}$ for $i=1, \ldots, m$, for any $s$ with $0<s<s^{*}$.

Furthermore, we assume that the number of operations required for evaluating each required entry in the tensor approximations of $\mathbf{A}$ or $\mathbf{f}$ is uniformly bounded.

Note that the requirement on $\mathbf{f}$ in (iii) is actually very mild because the data are typically more tensor sparse than the solution.

The following complexity estimates are formulated only for the more interesting case of the hierarchical Tucker format. Similar statements hold for the Tucker format, involving however additional terms that depend exponentially on $m$, which makes this format suitable only for moderate values of $m$.

Remark 11. Let $\mathbf{v}$ have finite support with finite ranks, i.e., $\operatorname{rank}_{\alpha}(\mathbf{v})<\infty$ for $\alpha \in$ $\mathcal{D}_{m}$. Then under Assumptions 1. APPLY can be realized numerically such that for $\mathbf{w}_{\eta}:=$ $\operatorname{APPLy}(\mathbf{v} ; \eta)$ we have (see Theorem 8 and Remark 7 )

$$
\begin{gathered}
\# \operatorname{supp}_{i}\left(\mathbf{w}_{\eta}\right) \lesssim C_{\mathbf{A}}^{\frac{1}{s}}\left(d_{\mathbf{A}}^{-1} \ln \left(M_{\mathbf{A}} / \eta\right)\right)^{\left(1+s^{-1}\right) b_{\mathbf{A}}}\left(\sum_{j=1}^{m}\left\|\pi^{(j)}(\mathbf{v})\right\|_{\mathcal{A}^{s}}\right)^{\frac{1}{s}} \eta^{-\frac{1}{s}} \\
\left\|\pi^{(i)}\left(\mathbf{w}_{\eta}\right)\right\|_{\mathcal{A}^{s}} \lesssim C_{\mathbf{A}}\left(d_{\mathbf{A}}^{-1} \ln \left(M_{\mathbf{A}} / \eta\right)\right)^{(s+1) b_{\mathbf{A}}}\left\|\pi^{(i)}(\mathbf{v})\right\|_{\mathcal{A}^{s}} \\
\left|\operatorname{rank}\left(\mathbf{w}_{\eta}\right)\right|_{\infty} \leq\left(d_{\mathbf{A}}^{-1} \ln \left(M_{\mathbf{A}} / \eta\right)\right)^{b_{\mathbf{A}}}|\operatorname{rank}(\mathbf{v})|_{\infty}
\end{gathered}
$$


and, by 108,

$$
\begin{aligned}
\operatorname{ops}\left(\mathbf{w}_{\eta}\right) \lesssim & (m-1)\left(d_{\mathbf{A}}^{-1} \ln \left(M_{\mathbf{A}} / \eta\right)\right)^{3 b_{\mathbf{A}}}|\operatorname{rank}(\mathbf{v})|_{\infty}^{3} \\
& +m C_{\mathbf{A}}^{\frac{1}{s}}\left(d_{\mathbf{A}}^{-1} \ln \left(M_{\mathbf{A}} / \eta\right)\right)^{\left(1+s^{-1}\right) b_{\mathbf{A}}}|\operatorname{rank}(\mathbf{v})|_{\infty}\left(\sum_{i=1}^{m}\left\|\pi^{(i)}(\mathbf{v})\right\|_{\mathcal{A}^{s}}\right)^{\frac{1}{s}} \eta^{-\frac{1}{s}}
\end{aligned}
$$

Thus, up to polylogarithmic terms, the curse of dimensionality is avoided. If in addition the approximations of $\mathbf{A}$ are equi-s*-compressible, the polylogarithmic terms in the above estimates improve according to Remark 8 .

Remark 12. Under Assumptions 1, the routine RHS can be realized numerically such that for $\mathbf{f}_{\eta}:=\operatorname{RHS}(\eta)$ we have

$$
\begin{gathered}
\# \operatorname{supp}_{i}\left(\mathbf{f}_{\eta}\right) \lesssim \eta^{-\frac{1}{s}}\left\|\pi^{(i)}(\mathbf{f})\right\|_{\mathcal{A}^{s}}^{\frac{1}{s}} \\
\left\|\pi^{(i)}\left(\mathbf{f}_{\eta}\right)\right\|_{\mathcal{A}^{s}} \lesssim\left\|\pi^{(i)}(\mathbf{f})\right\|_{\mathcal{A}^{s}}, \\
\left|\operatorname{rank}\left(\mathbf{f}_{\eta}\right)\right|_{\infty} \lesssim\left(d_{\mathbf{f}}^{-1} \ln \left(\|\mathbf{f}\|_{\mathcal{A}_{\mathcal{H}}\left(\gamma_{\mathbf{f}}\right)} / \eta\right)\right)^{b_{\mathbf{f}}}
\end{gathered}
$$

as well as

$$
\operatorname{ops}\left(\mathbf{f}_{\eta}\right) \lesssim(m-1)\left|\operatorname{rank}\left(\mathbf{f}_{\eta}\right)\right|_{\infty}^{3}+\left|\operatorname{rank}\left(\mathbf{f}_{\eta}\right)\right|_{\infty} \sum_{i=1}^{m} \# \operatorname{supp}_{i}\left(\mathbf{f}_{\eta}\right) .
$$

Remark 13. We take RECOMPRESS as a numerical realization of $\hat{\mathrm{P}}_{\eta}$ as defined in (43). This amounts to the computation of an HOSVD or $\mathcal{H} S V D$, respectively, for which we have the complexity bounds given in Remarks 1 and 3 .

Likewise, COARSEN is a numerical realization of $\hat{\mathrm{C}}_{\eta}$ as defined in (66), with the modification of replacing the exact sorting of the values $\pi_{\nu_{i}}^{(i)}(\cdot), i=1, \ldots, m, \nu \in \nabla^{d_{i}}$, as required by $\hat{\mathrm{C}}_{\eta}$, by an approximate sorting as proposed in [4, 26], see Remark 9. This leads to an increase of $\kappa_{\mathrm{C}}$ by only a fixed factor; for finitely supported $\mathbf{v}$, the procedure can be realized in practice such that $\kappa_{\mathrm{C}}=2 \sqrt{m}$, and using a number of operations bounded by

$$
C|\operatorname{rank}(\mathbf{v})|_{\infty} \sum_{i=1}^{m} \# \operatorname{supp}_{i}(\mathbf{v})
$$

with a fixed $C>0$. Note that here we make the implicit assumption that the orthogonality properties required by COARSEN have been enforced if necessary before the application of COARSEN. This can be done by an application of $\operatorname{ReCOMPRESS}(\cdot, 0)$.

Note that under the assumptions of Proposition 5, the iteration converges for any fixed $\beta \geq 0$. A call to RECOMPRESS (possibly with $\beta=0$, i.e., without performing an approximation) is in fact necessary in each inner iteration to ensure the orthogonality properties required by APPLY.

The main result of this paper is the following theorem. It says that whenever the solution has the approximation properties specified in Assumptions 1, then the adaptive scheme recovers these rates and the required computational work has optimal complexity up to logarithmic factors. We have made an attempt to identify the dependencies of the involved constants on the problem parameters as explicitly as possible. 
Theorem 9. Let $\alpha>0$ and let $\kappa_{\mathrm{P}}, \kappa_{\mathrm{C}}$ be as in Theorem 17. Let the constants $\kappa_{1}, \kappa_{2}, \kappa_{3}$ in Algorithm 5.1 be chosen as

$$
\begin{gathered}
\kappa_{1}=\left(1+(1+\alpha)\left(\kappa_{\mathrm{P}}+\kappa_{\mathrm{C}}+\kappa_{\mathrm{P}} \kappa_{\mathrm{C}}\right)\right)^{-1}, \\
\kappa_{2}=(1+\alpha) \kappa_{\mathrm{P}} \kappa_{1}, \quad \kappa_{3}=\kappa_{\mathrm{C}}\left(\kappa_{\mathrm{P}}+1\right)(1+\alpha) \kappa_{1} .
\end{gathered}
$$

Let $\mathbf{A u}=\mathbf{f}$, where $\mathbf{A}, \mathbf{u}, \mathbf{f}$ satisfy Assumptions 1 . Then $\mathbf{u}_{\varepsilon}$ produced by Algorithm 5.1 satisfies

$$
\begin{gathered}
\left|\operatorname{rank}\left(\mathbf{u}_{\varepsilon}\right)\right|_{\infty} \leq\left(d_{\mathbf{u}}^{-1} \ln \left[(\theta \alpha)^{-1} \rho_{\gamma_{\mathbf{u}}}\|\mathbf{u}\|_{\mathcal{A}_{\mathcal{H}}\left(\gamma_{\mathbf{u}}\right)} \varepsilon^{-1}\right]\right)^{b_{\mathbf{u}}}, \\
\sum_{i=1}^{m} \# \operatorname{supp}_{i}\left(\mathbf{u}_{\varepsilon}\right) \lesssim\left(\sum_{i=1}^{m}\left\|\pi^{(i)}(\mathbf{u})\right\|_{\mathcal{A}^{s}}\right)^{\frac{1}{s}} \varepsilon^{-\frac{1}{s}}
\end{gathered}
$$

as well as

$$
\begin{aligned}
\left\|\mathbf{u}_{\varepsilon}\right\|_{\mathcal{A}_{\mathcal{H}}\left(\gamma_{\mathbf{u}}\right)} & \lesssim\|\mathbf{u}\|_{\mathcal{A}_{\mathcal{H}}\left(\gamma_{\mathbf{u}}\right)}, \\
\sum_{i=1}^{m}\left\|\pi^{(i)}\left(\mathbf{u}_{\varepsilon}\right)\right\|_{\mathcal{A}^{s}} & \lesssim \sum_{i=1}^{m}\left\|\pi^{(i)}(\mathbf{u})\right\|_{\mathcal{A}^{s}}
\end{aligned}
$$

The multiplicative constant in (122) depends only on $\alpha$ and $m$, those in (121) and (123) depend only on $\alpha, m$ and $s$. For the number of required operations, we have the estimate

$$
\operatorname{ops}\left(\mathbf{u}_{\varepsilon}\right) \lesssim|\ln \varepsilon|^{J\left(3+s^{-1}\right) b_{\mathbf{A}}+2 b_{\mathbf{f}}}\left(\sum_{i=1}^{m} \max \left\{\left\|\pi^{(i)}(\mathbf{u})\right\|_{\mathcal{A}^{s}},\left\|\pi^{(i)}(\mathbf{f})\right\|_{\mathcal{A}^{s}}\right\}\right)^{\frac{1}{s}} \varepsilon^{-\frac{1}{s}}
$$

with a multiplicative constant independent of $\varepsilon$ and $\left\|\pi^{(i)}(\mathbf{u})\right\|_{\mathcal{A}^{s}},\left\|\pi^{(i)}(\mathbf{f})\right\|_{\mathcal{A}^{s}}$, and with an algebraic explicit dependence on $m$ and $C_{\mathbf{A}}$.

Remark 14. The maximum number of inner iterations $J$ that arises in the complexity estimate is defined in line 2 of Algorithm 5.1. This value depends on the freely chosen algorithm parameters $\beta$ and $\theta$, on the constants $\omega$ and $\rho$ that depend only on $\mathbf{A}$, and on $\kappa_{1}$. Thus, $J$ depends on $m$ : The choice of $\kappa_{1}$ in Theorem 9 leads to $\kappa_{1} \sim m^{-1}$, and hence $J \sim \log m$. Note that since $|\ln \varepsilon|^{c \ln m}=m^{c \ln |\ln \varepsilon|}$, this leads to an algebraic dependence of the complexity estimate on $m$. Furthermore, the precise dependence of the constant in (124) on $m$ is also influenced by the problem parameters from Assumption 1, which may contain additional implicit dependencies on $m$. In particular, as can be seen from the proof, the constant has a linear dependence on $C_{\mathbf{A}}^{J / s}$ if $C_{\mathbf{A}}>1$ (cf. Remark 6).

Proof of Theorem 9. By the choice of $\kappa_{1}, \kappa_{2}, \kappa_{3}$, we can apply Lemma 7 to each $\mathbf{u}_{i}$ produced in line 11 of Algorithm 5.1, which yields the bounds (120), (121), (122), (123) for the values $\varepsilon=\theta^{k} \delta, k \in \mathbb{N}$.

It therefore remains to estimate the computational complexity of each inner loop. Note that RECOMPRESS in line 8 does not deteriorate the approximability of the intermediates $\mathbf{w}_{j}$ as a consequence of Lemma 3 .

Let $\varepsilon_{k}:=\theta^{k} \delta$. We already know from Theorem 7 that

$$
\begin{aligned}
\left|\operatorname{rank}\left(\mathbf{u}_{k}\right)\right|_{\infty} & \leq\left(d_{\mathbf{u}}^{-1} \ln \left[\alpha^{-1} \rho_{\gamma_{\mathbf{u}}}\|\mathbf{u}\|_{\mathcal{A}_{\mathcal{H}}\left(\gamma_{\mathbf{u}}\right)} \varepsilon_{k}^{-1}\right]\right)^{b_{\mathbf{u}}} \lesssim\left|\ln \varepsilon_{k}\right|^{b_{\mathbf{u}}}, \\
\sum_{i=1}^{m} \# \operatorname{supp}_{i}\left(\mathbf{u}_{k}\right) & \lesssim\left(\sum_{i=1}^{m}\left\|\pi^{(i)}(\mathbf{u})\right\|_{\mathcal{A}^{s}}\right)^{\frac{1}{s}} \varepsilon_{k}^{-\frac{1}{s}} \\
\sum_{i=1}^{m}\left\|\pi^{(i)}\left(\mathbf{u}_{k}\right)\right\|_{\mathcal{A}^{s}} & \lesssim \sum_{i=1}^{m}\left\|\pi^{(i)}(\mathbf{u})\right\|_{\mathcal{A}^{s}}
\end{aligned}
$$


where the multiplicative constants in the last two equations depend on $\alpha, m$, and $s$. Similarly, we obtain (122) from (70). Furthermore, by definition of the iteration,

$$
\left|\operatorname{rank}\left(\mathbf{w}_{j+1}\right)\right|_{\infty} \leq\left(d_{\mathbf{A}}^{-1} \ln \left(2 M_{\mathbf{A}} / \eta_{j}\right)\right)^{b_{\mathbf{A}}}\left|\operatorname{rank}\left(\mathbf{w}_{j}\right)\right|_{\infty}+\left(d_{\mathbf{f}}^{-1} \ln \left(2|\mathbf{f}|_{\mathcal{A}_{\mathcal{H}}\left(\gamma_{\mathbf{f}}\right)} / \eta_{j}\right)\right)^{b_{\mathbf{f}}} .
$$

Combining this with 125 and using $b_{\mathbf{f}}>b_{\mathbf{u}}$, we obtain

$$
\left|\operatorname{rank}\left(\mathbf{w}_{j}\right)\right|_{\infty} \lesssim\left|\ln \varepsilon_{k}\right|^{j b_{\mathbf{A}}+b_{\mathbf{f}}} .
$$

The definition of the iterates also yields

$$
\begin{aligned}
\# \operatorname{supp}_{i}\left(\mathbf{w}_{j+1}\right) & \lesssim \# \operatorname{supp}_{i}\left(\mathbf{w}_{j}\right) \\
\qquad C_{\mathbf{A}}^{\frac{1}{s}}\left(d_{\mathbf{A}}^{-1} \ln \left(2 M_{\mathbf{A}} / \eta_{j}\right)\right)^{\left(1+s^{-1}\right) b_{\mathbf{A}}}\left(\sum_{l=1}^{m}\left\|\pi^{(l)}\left(\mathbf{w}_{j}\right)\right\|_{\mathcal{A}^{s}}\right)^{\frac{1}{s}} \eta_{j}^{-\frac{1}{s}} & +\left\|\pi^{(i)}(\mathbf{f})\right\|_{\mathcal{A}^{s}}^{\frac{1}{s}} \eta_{j}^{-\frac{1}{s}}
\end{aligned}
$$

and by 113 ,

$$
\begin{aligned}
\left\|\pi^{(i)}\left(\mathbf{w}_{j}\right)\right\|_{\mathcal{A}^{s}} \lesssim\left\|\pi^{(i)}\left(\mathbf{w}_{j-1}\right)\right\|_{\mathcal{A}^{s}} & \\
& +\omega C_{\mathbf{A}}\left(d_{\mathbf{A}}^{-1} \ln \left(2 M_{\mathbf{A}} / \eta_{j-1}\right)\right)^{(1+s) b_{\mathbf{A}}}\left\|\pi^{(i)}\left(\mathbf{w}_{j-1}\right)\right\|_{\mathcal{A}^{s}}+\omega\left\|\pi^{(i)}(\mathbf{f})\right\|_{\mathcal{A}^{s}} .
\end{aligned}
$$

Using these estimates recursively together with (127), (126), we obtain

$$
\left\|\pi^{(i)}\left(\mathbf{w}_{j}\right)\right\|_{\mathcal{A}^{s}} \lesssim\left|\ln \varepsilon_{k}\right|^{j(1+s) b_{\mathbf{A}}} \max \left\{\left\|\pi^{(i)}(\mathbf{u})\right\|_{\mathcal{A}^{s}},\left\|\pi^{(i)}(\mathbf{f})\right\|_{\mathcal{A}^{s}}\right\}
$$

and

$$
\sum_{i=1}^{m} \# \operatorname{supp}_{i}\left(\mathbf{w}_{j}\right) \lesssim\left|\ln \varepsilon_{k}\right|^{j\left(1+s^{-1}\right) b_{\mathbf{A}}}\left(\sum_{i=1}^{m} \max \left\{\left\|\pi^{(i)}(\mathbf{u})\right\|_{\mathcal{A}^{s}},\left\|\pi^{(i)}(\mathbf{f})\right\|_{\mathcal{A}^{s}}\right\}\right)^{\frac{1}{s}} \varepsilon_{k}^{-\frac{1}{s}}
$$

The total number of operations for the calls of APPLY in an inner loop according to (115) is dominated by that for the calls of RECOMPRESS, which can be bounded up to a constant by

$$
m\left|\operatorname{rank}\left(\mathbf{w}_{J}\right)\right|_{\infty}^{4}+\left|\operatorname{rank}\left(\mathbf{w}_{J}\right)\right|_{\infty}^{2} \sum_{i=1}^{m} \# \operatorname{supp}_{i}\left(\mathbf{w}_{J}\right) .
$$

We thus arrive at (124).

Remark 15. The above results apply directly to problems posed on separable tensor product Hilbert spaces, for which tensor product Riesz bases are available. Note, however, that this is not the case for standard Sobolev spaces $\mathrm{H}^{s}\left(\Omega^{d}\right)$, which are not tensor product spaces themselves, but, for tensor product domains $\Omega^{d}$, can be represented as intersections of $d$ tensor product spaces.

As mentioned in the introduction, from a sufficiently regular tensor product wavelet basis $\left\{\Psi_{\nu}:=\psi_{\nu_{1}} \otimes \cdots \otimes \psi_{\nu_{d}}\right\}_{\nu \in \nabla^{d}}$ of $\mathrm{L}_{2}\left(\Omega^{d}\right)$, we can obtain a Riesz basis of $\mathrm{H}^{s}\left(\Omega^{d}\right)$ by a level-dependent rescaling of basis functions, e.g.,

$$
\left\{2^{-s \max _{i}\left|\nu_{i}\right|} \Psi_{\nu}\right\}_{\nu \in \nabla^{d}}
$$


To again arrive at a problem on $\ell_{2}$, we now rewrite the original operator equation $A u=f$, with $A: \mathrm{H}^{s}\left(\Omega^{d}\right) \rightarrow\left(\mathrm{H}^{s}\left(\Omega^{d}\right)\right)^{\prime}$, in the form

$$
\begin{aligned}
\sum_{\mu \in \nabla^{d}}\left(2^{-s\left(\max _{i}\left|\nu_{i}\right|+\max _{i}\left|\mu_{i}\right|\right)}\left\langle A \Psi_{\mu}, \Psi_{\nu}\right\rangle\right)\left(2^{s \max _{i}\left|\mu_{i}\right|}\left\langle u, \Psi_{\mu}\right\rangle\right) & \\
& =2^{-s\left(\max _{i}\left|\nu_{i}\right|\right.}\left\langle f, \Psi_{\nu}\right\rangle, \quad \nu \in \nabla^{d} .
\end{aligned}
$$

We thus obtain a well-posed problem on $\ell_{2}\left(\nabla^{d}\right)$ for the rescaled coefficient sequence $\mathbf{u}=$ $2^{s \max _{i}\left|\mu_{i}\right|}\left\langle u, \Psi_{\mu}\right\rangle$ and the infinite matrix $\mathbf{A}=2^{-s\left(\max _{i}\left|\nu_{i}\right|+\max _{i}\left|\mu_{i}\right|\right)}\left\langle A \Psi_{\mu}, \Psi_{\nu}\right\rangle$.

This diagonal rescaling, which in the case of finite-dimensional Galerkin approximations corresponds to a preconditioning of A, leads to additional problems in our context: the sequence $\left(2^{-s \max _{i}\left|\nu_{i}\right|}\right)_{\nu \in \nabla^{d}}$ (as well as possible equivalent alternatives) has infinite rank on the full index set $\nabla^{d}$. As a consequence, the ranks in an approximation of $\mathbf{A} \mathbf{v}$ for some given $\mathbf{v}$ will in general depend on the range of values of $\left|\nu_{1}\right|, \ldots,\left|\nu_{d}\right|$ for the nonzero coefficients $\mathbf{v}_{\nu_{1}, \ldots, \nu_{d}}$. This issue and possible strategies for handling it are discussed in more detail in [2]. The complexity analysis of iterative schemes in the case of $\mathbf{A}$ involving such a rescaling will be treated in a separate publication.

\section{$6 \quad$ Numerical Experiments}

We choose our example to illustrate the results of the previous section numerically according to several criteria. In order to arrive at a valid comparison between different dimensions, we choose a problem on $\mathrm{L}_{2}\left([0,1]^{d}\right)$ that has similar properties for different values of $d$. The problem has a discontinuous right hand side and solution, which means that reasonable convergence rates can be achieved only by adaptive approximation. It is also still sufficiently simple such that all constants used in Algorithm 5.1 can be chosen rigorously according to the requirements of the convergence analysis.

We set $\Omega:=[0,1]^{d}$ and use tensor order $m=d$. As an orthonormal wavelet basis $\left\{\psi_{\nu}\right\}_{\nu \in \nabla}$ of $\mathrm{L}_{2}([0,1])$, we use Alpert multiwavelets [1] of polynomial order $p \in \mathbb{N}$. Let

$$
(T v)(t):=\int_{0}^{t} v \mathrm{~d} s
$$

then $T$ is a compact operator on $\mathrm{L}_{2}([0,1])$ with $\|T\|=2 / \pi$. The infinite matrix representation $\left(\left\langle T \psi_{\mu}, \psi_{\nu}\right\rangle\right)_{\nu, \mu \in \nabla}$ is $s^{*}$-compressible for any $s^{*}>0$.

For $f \in \mathrm{L}_{2}(\Omega)$, we consider the integral equation

$$
\left(\mathrm{I}-\omega_{d} \bigotimes_{i=1}^{d} T\right) u=f
$$

with $\omega_{d}=\frac{1}{2}\left(\frac{\pi}{2}\right)^{d}$. Note that for $B:=\omega_{d} \bigotimes_{i=1}^{d} T$ and $A:=\mathrm{I}-B$ we have $\|B\|=\frac{1}{2}$, and therefore

$$
A^{-1}=(\mathrm{I}-B)^{-1}=\sum_{k=0}^{\infty} B^{k}=\sum_{k=0}^{\infty} \omega_{d}^{k} \bigotimes_{i=1}^{d} T^{k} .
$$

Furthermore, $A:=\mathrm{I}-B$ is a nonsymmetric, $\mathrm{L}_{2}$-elliptic operator with $\langle A v, v\rangle \geq \frac{1}{2}\|v\|_{\mathrm{L}_{2}(\Omega)}^{2}$ as well as $\|A\| \leq \frac{3}{2}$. Since $\mathbf{A}$ is the representation with respect to an orthonormal basis, we obtain $\lambda_{\mathbf{A}}=\frac{1}{2}$ and $\Lambda_{\mathbf{A}}=\frac{3}{2}$. Due to the special structure of the operator, choosing the 
iteration parameter $\omega$ as $\omega:=1$, we have $\|\mathrm{I}-\omega \mathbf{A}\| \leq \frac{1}{2}=: \rho$. We choose the right hand side as

$$
f=(1-\tau) \sum_{k=0}^{\infty} \bigotimes_{i=1}^{d} \tau^{k} f_{k}, \quad f_{k}(x):=\sqrt{2 \pi} \chi_{[0,1 / \pi]} \cos \left(2 \pi^{2}(k+1) x\right),
$$

where $\tau \in(0,1)$. This gives $\left\|f_{k}\right\|_{\mathrm{L}_{2}([0,1])}=1$ and $\|f\|_{\mathrm{L}_{2}(\Omega)}=\|\mathbf{f}\|=1$, and $\pi^{(i)}(\mathbf{f}) \in \mathcal{A}^{s}$ for any $s>0$. The functions $f_{k}$ have jump discontinuities at $\pi^{-1}$, which need to be resolved adaptively in order to maintain the optimal approximation rate for the given wavelet basis.

From the expansion for $(\mathrm{I}-B)^{-1}$, we already know that $\pi^{(i)}(\mathbf{u}) \in \mathcal{A}^{s}$ for any $s<p$, for $i=1, \ldots, m$. We also have the explicit representation

$$
u=(1-\tau) \sum_{k, n=0}^{\infty} \tau^{k} \omega_{d}^{n} \bigotimes_{i=1}^{d} T^{n} f_{k}
$$

For the choice of $f_{k}$ under consideration, evaluating $\omega_{d}^{n} \bigotimes_{i=1}^{d} T^{n} f_{k}$, we obtain $u \rightarrow f$ as $d \rightarrow \infty$; that is, the mode singular values of the solution approach exponential decay with rate $\tau$ for growing $d$. Since $\|u-f\|_{\mathrm{L}_{2}}$ is small for any $d>3$, $\mathbf{u}$ has similar low-rank approximability for all relevant $d$.

Hence for our particular choice of $f$, the action of $A^{-1}$ is close to the identity. It should be emphasized, however, that this only simplifies the interpretation of the results, but does not simplify the problem from a computational point of view, since our algorithm does not make use of this particularity. We have also chosen a problem that is completely symmetric with respect to all variables to simplify the tests and the comparison between values of $d$, but do not make computational use of this symmetry.

For the further constants arising in the iteration, we choose $\theta:=\frac{1}{2}$ and $\beta:=1$. For the hierarchical Tucker format, we have $\kappa_{\mathrm{P}}=\sqrt{2 m-3}$ and $\kappa_{\mathrm{C}}=\sqrt{m}$, and fix the derived constants $\kappa_{1}, \kappa_{2}, \kappa_{3}$ as in Theorem 9 by taking $\alpha:=1$. Furthermore, we have $\delta=\lambda_{\mathbf{A}}^{-1}\|\mathbf{f}\|=2$.

Remark 16. Since many steps of the algorithm - including the comparably expensive approximate application of lower-dimensional operators to tensor factors and $Q R$ factorizations of mode frames - can be done independently for each mode, an effective parallelization of our adaptive scheme is quite easy to achieve.

In all following examples, we use piecewise cubic wavelets. The implementation was done in $\mathrm{C}++$ using standard LAPACK routines for linear algebra operations. Iterations are stopped as soon as a required wavelet index cannot be represented as a signed 64-bit integer.

We make some simplifications in counting the number of required operations: For each matrix-matrix product, $Q R$ factorization, and SVD, we use the standard estimates for the required number of multiplications (see, e.g., [18]); for the approximation of $\mathbf{A}$ and f, we count one operation per multiplication with a matrix entry and per generated right hand side entry, respectively (note that we thus make the simplifying assumption that all required wavelet coefficients can be evaluated using $\mathcal{O}(1)$ operations, which could in principle be realized in the present example, but is not strictly satisfied in our current implementation). We thus neglect some minor contributions that do not play any asymptotic role, such as the number of operations required for adding two tensor representations, and the sorting of tensor contraction values for COARSEN, which here is done by a standard library call for simplicity. 


\subsection{Results with Right Hand Side of Rank 1}

For comparison, we first consider a simplified version of the right hand side reduced to the first summand, that is,

$$
f=\bigotimes_{i=1}^{d} \sqrt{2 \pi} \chi_{[0,1 / \pi]} \cos \left(2 \pi^{2} \cdot\right) .
$$

In high dimensions, the solution $u$ coincides with $f$ up to very small correction terms.
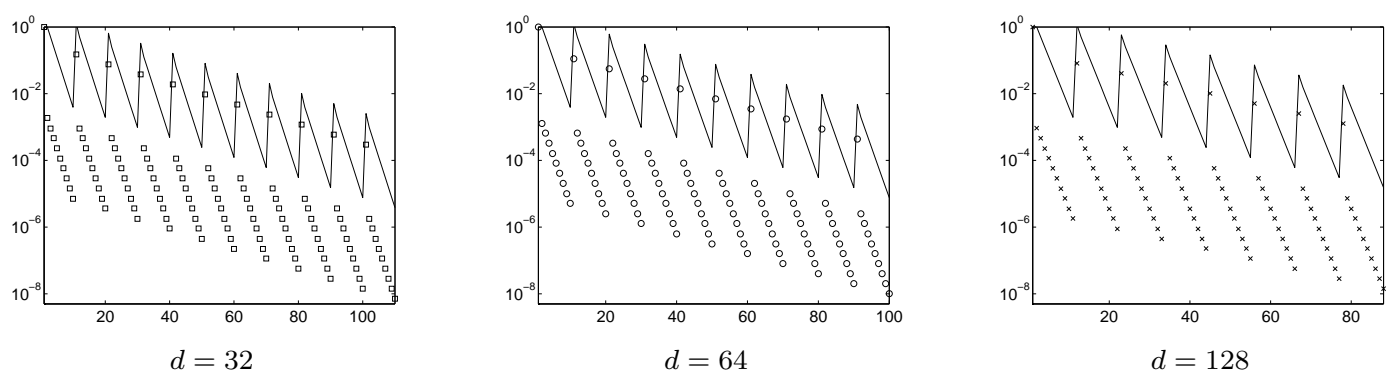

Figure 1: Computed approximate residual norms (markers) and corresponding solution error estimates (solid lines), for $f$ of rank one.

The evolution of the computed approximate residual norms and the corresponding estimates for the $\mathrm{L}_{2}$-deviation from the solution of the infinite-dimensional problem is shown in Figure 1. Here one can clearly observe the effect of the coarsening steps after a certain number of inner iterations. Apart from the expected increase in the number $J$ of such inner iterations with dimension, the iteration shows quite similar behaviour for different $d$. In particular, in each case the resulting iterates $\mathbf{w}_{j}$ in Algorithm 5.1 have rank 1 , the residuals $\mathbf{r}_{j}$ have ranks at most 3 , thus the maximum rank arising in the iteration is 4 .

Note that the iteration is stopped a few steps earlier with increasing dimension because slightly stricter error tolerances are applied in the approximation of operator and right hand side. This means that the technical limit for the maximum possible wavelet level is reached earlier.

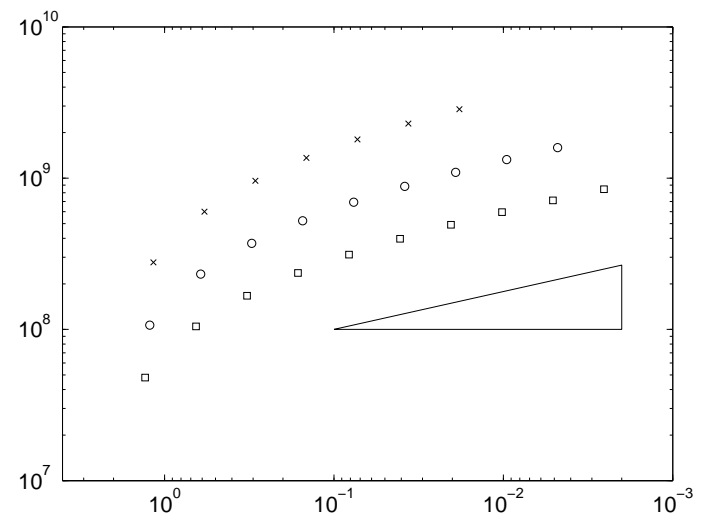

Figure 2: Total operation count at the end of each inner iteration in dependence on the estimated error $(\square d=32, \circ d=64, \times d=128)$, for $f$ of rank one. The triangle shows a slope of $\frac{1}{4}$. 
We see that the number of operations, shown in Figure 2, increases at a rate close to the approximation order 4 of our wavelet basis. What is most remarkable here, however, is the very mild - almost linear - dependence of the total complexity on the dimension: a doubling of dimension leads to only slightly more than twice the number of operations.

\subsection{Results with Right Hand Side of Unbounded Rank}

We now use the full right hand side $f$ as in $(129)$, which leads to a solution with unbounded rank, and approximately the same exponential decay of singular values as $f$.
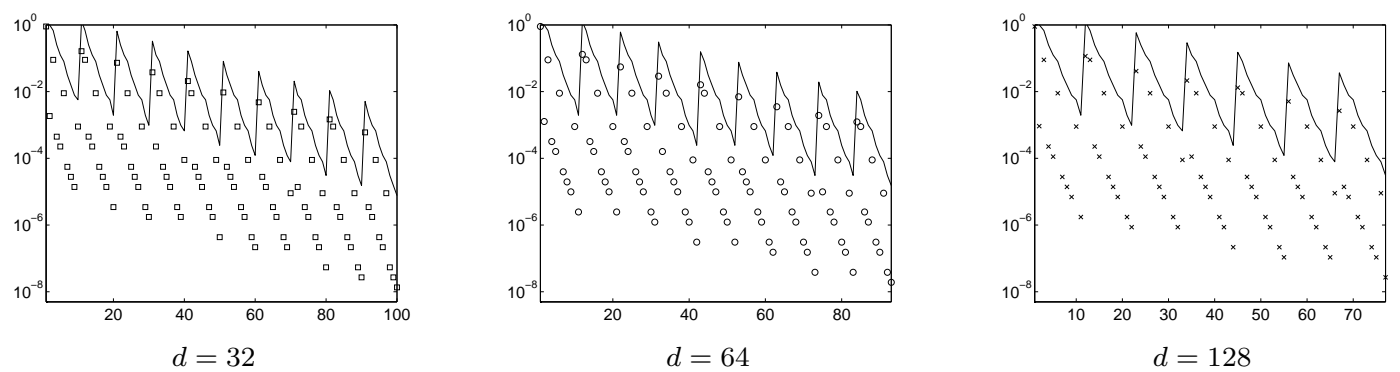

Figure 3: Computed approximate residual norms (markers) and corresponding solution error estimates (solid lines), for $f$ of unbounded rank.

As shown in Figure 3 , the computed residual estimates and the corresponding estimates for the solution error behave quite similarly to the previous example. In the present case, the computed residual norms show a less regular pattern, which is mostly due to the adjustment of approximation ranks for the right hand side.
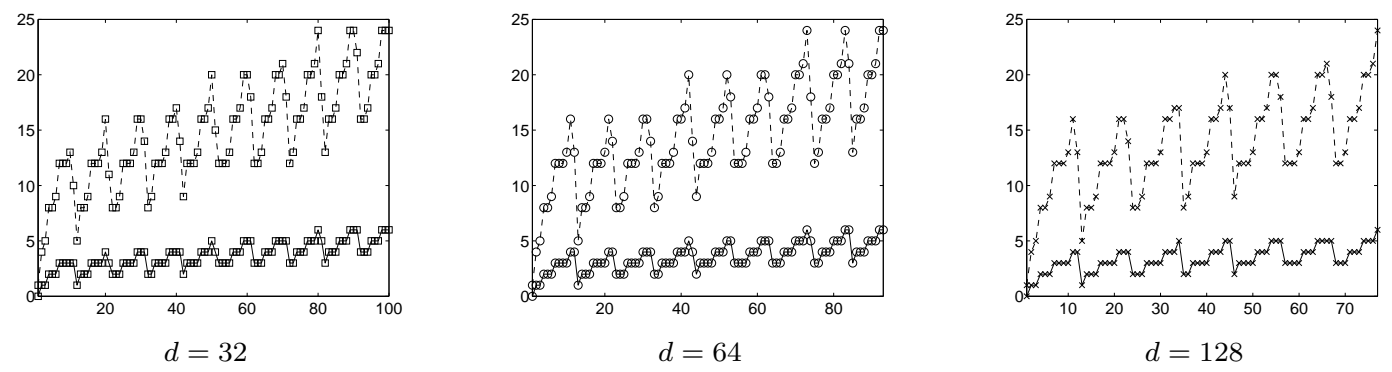

Figure 4: Maximum ranks of iterates $\mathbf{w}_{j}$ (solid lines) and of maximum rank of all intermediates arising in the inner iteration steps (dashed lines), for $f$ of unbounded rank.

The ranks of the produced iterates $\mathbf{w}_{j}$, as well as those of the intermediate quantities arising in the iteration (see line 8 of Algorithm 5.1 prior to the recompression operation), shows a steady but controlled increase during the iteration, as shown in Figure 4 .

Note that in this case, the number of operations, shown in Figure 5 , increases visibly faster than the limiting rate corresponding to the approximation order of the lowerdimensional multiresolution spaces. Due to the higher tensor ranks involved, this is to be expected in view of our complexity estimates. The increase of complexity with the problem dimension, however, still remains very moderate. 


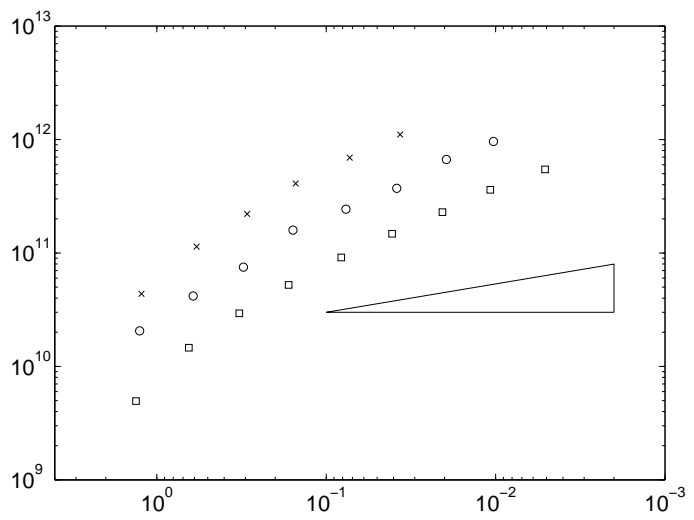

Figure 5: Operation count at the end of each inner iteration in dependence on the estimated error $(\square d=32, \circ d=64, \times d=128)$, for $f$ of unbounded rank. The triangle shows a slope of $\frac{1}{4}$.

\section{Conclusion and Outlook}

The presented theory and examples indicate that the schemes developed in this work can be applied to very high-dimensional problems, with a rigorous foundation for the type of elliptic operator equations considered here. The results can be extended to more general operator equations, as long as the variational formulation, in combination with a suitable basis, induces a well-conditioned isomorphism on $\ell_{2}$. However, when the operator represents an isomorphism between spaces that are not simple tensor products, such as Sobolev spaces and their duals, additional concepts are required, which will be developed in a subsequent publication.

\section{References}

[1] B. Alpert, A class of bases in $L^{2}$ for the sparse representation of integral operators, SIAM J. Math. Anal., 24 (1991), pp. 246-262.

[2] M. Bachmayr, Adaptive Low-Rank Wavelet Methods and Applications to TwoElectron Schrödinger Equations, PhD thesis, RWTH Aachen, 2012.

[3] J. Ballani And L. Grasedyck, A projection method to solve linear systems in tensor format, Numerical Linear Algebra with Applications, 20 (2013), pp. 27-43.

[4] A. Barinka, Fast Evaluation Tools for Adaptive Wavelet Schemes, PhD thesis, RWTH Aachen, 2005.

[5] G. Beylkin and M. J. Mohlenkamp, Numerical operator calculus in higher dimensions, PNAS, 99 (2002), pp. 10246-10251.

[6] G. Beylkin and M. J. Mohlenkamp, Algorithms for numerical analysis in high dimensions, SIAM J. Sci. Comput., 26 (2005), pp. 2133-2159.

[7] A. Cohen, Numerical Analysis of Wavelet Methods, vol. 32 of Studies in Mathematics and Its Applications, Elsevier, 2003. 
[8] A. Cohen, W. Dahmen, And R. DeVore, Adaptive wavelet methods for elliptic operator equations: Convergence rates, Mathematics of Computation, 70 (2001), pp. $27-75$.

[9] A. Cohen, W. Dahmen, And R. DeVore, Adaptive wavelet methods II - beyond the elliptic case, Foundations of Computational Mathematics, 2 (2002), pp. 203-245.

[10] V. DE Silva AND L.-H. Lim, Tensor rank and the ill-posedness of the best lowrank approximation problem, SIAM Journal on Matrix Analysis and Applications, 30 (2008), pp. 1084-1127.

[11] R. DeVore, G. Petrova, and P. Wojtaszczyk, Approximation of functions of few variables in high dimensions, Constructive Approximation, 33 (2011), pp. 125143.

[12] T. J. Dijkema, C. Schwab, And R. Stevenson, An adaptive wavelet method for solving high-dimensional elliptic PDEs, Constructive Approximation, 30 (2009), pp. $423-455$.

[13] A. Falcó and W. Hackbusch, On minimal subspaces in tensor representations, Foundations of Computational Mathematics, 12 (2012), pp. 765-803.

[14] A. FAlcó AND A. Nouy, Proper generalized decomposition for nonlinear convex problems in tensor banach spaces, Numerische Mathematik, 121 (2012), pp. 503-530.

[15] L. GRASEDYCK, Hierarchical singular value decomposition of tensors, SIAM J. Matrix Anal. Appl., 31 (2010), pp. 2029-2054.

[16] L. Grasedyck, D. Kressner, and C. Tobler, A literature survey of low-rank tensor approximation techniques, tech. rep., arXiv:1302.7121 [math.NA], 2013.

[17] M. Griebel and H. Harbrecht, Approximation of two-variate functions: Singular value decomposition versus regular sparse grids. INS Preprint No. 1109, Universität Bonn, 2011.

[18] W. Hackbusch, Tensor Spaces and Numerical Tensor Calculus, vol. 42 of Springer Series in Computational Mathematics, Springer-Verlag Berlin Heidelberg, 2012.

[19] W. Hackbusch, B. Khoromskij, and E. Tyrtyshnikov, Approximate iterations for structured matrices, Numerische Mathematik, 109 (2008), pp. 119-156.

[20] W. Hackbusch And S. KüHn, A new scheme for the tensor representation, Journal of Fourier Analysis and Applications, 15 (2009), pp. 706-722.

[21] B. N. KhoromskiJ And C. SchwaB, Tensor-structured Galerkin approximation of parametric and stochastic elliptic PDEs, SIAM J. Sci. Comput., 33 (2011), pp. 364385 .

[22] T. G. Kolda AND B. W. BADER, Tensor decompositions and applications, SIAM Review, 51 (2009), pp. 455-500.

[23] D. Kressner And C. ToBler, Preconditioned low-rank methods for highdimensional elliptic PDE eigenvalue problems, Computational Methods in Applied Mathematics, 11 (2011), pp. 363-381. 
[24] L. D. Lathauwer, B. D. Moor, And J. Vandewalle, A multilinear singular value decomposition, SIAM Journal on Matrix Analysis and Applications, 21 (2000), pp. $1253-1278$.

[25] H. G. Matthies And E. Zander, Solving stochastic systems with low-rank tensor compression, Linear Algebra and its Applications, 436 (2012), pp. 3819-3838.

[26] A. MetselaAR, Handling Wavelet Expansions in Numerical Methods, PhD thesis, University of Twente, 2002.

[27] E. Novak and H. Wozniakowski, Approximation of infinitely differentiable multivariate functions is intractable, Journal of Complexity, 25 (2009), pp. 398-404.

[28] I. Oseledets And E. Tyrtyshnikov, Breaking the curse of dimensionality, or how to use SVD in many dimensions, SIAM Journal on Scientific Computing, 31 (2009), pp. $3744-3759$.

[29] —_, Tensor tree decomposition does not need a tree, tech. rep., RAS Moscow 200908, 2009.

[30] I. V. Oseledets, Tensor-train decomposition, SIAM Journal on Scientific Computing, 33 (2011), pp. 2295-2317.

[31] R. Schneider And A. Uschmajew, Approximation rates for the hierarchical tensor format in periodic Sobolev spaces. MATHICSE Technical Report Nr. 06.2013, EPFL Lausanne, 2013.

[32] R. Stevenson, On the compressibility of operators in wavelet coordinates, SIAM Journal on Mathematical Analysis, 35 (2004), pp. 1110-1132.

[33] L. R. Tucker, Contributions to Mathematical Psychology, Holt, Rinehart \& Winston, New York, 1964, ch. The extension of factor analysis to three-dimensional matrices, pp. 109-127.

[34] _ Some mathematical notes on three-mode factor analysis, Psychometrika, 31 (1966), pp. 279-311.

[35] A. Uschmajew, Well-posedness of convex maximization problems on Stiefel manifolds and orthogonal tensor product approximations, Numerische Mathematik, 115 (2010), pp. 309-331.

[36] _ Regularity of tensor product approximations to square integrable functions, Constructive Approximation, 34 (2011), pp. 371-391. 